Article

\title{
Predictive Modeling of a Paradigm Mechanical Cooling Tower Model: II. Optimal Best-Estimate Results with Reduced Predicted Uncertainties
}

\author{
Ruixian Fang, Dan Gabriel Cacuci * and Madalina Badea \\ Center for Nuclear Science and Energy, Department of Mechanical Engineering, University of South Carolina, \\ Columbia, SC 29208, USA; fangr@cec.sc.edu (R.F.); Badea@cec.sc.edu (M.B.) \\ * Correspondence: cacuci@cec.sc.edu; Tel.: +1-803-777-9751
}

Academic Editor: Erich Schneider

Received: 23 June 2016; Accepted: 5 September 2016; Published: 16 September 2016

\begin{abstract}
This work uses the adjoint sensitivity model of the counter-flow cooling tower derived in the accompanying PART I to obtain the expressions and relative numerical rankings of the sensitivities, to all model parameters, of the following model responses: (i) outlet air temperature; (ii) outlet water temperature; (iii) outlet water mass flow rate; and (iv) air outlet relative humidity. These sensitivities are subsequently used within the "predictive modeling for coupled multi-physics systems" (PM_CMPS) methodology to obtain explicit formulas for the predicted optimal nominal values for the model responses and parameters, along with reduced predicted standard deviations for the predicted model parameters and responses. These explicit formulas embody the assimilation of experimental data and the "calibration" of the model's parameters. The results presented in this work demonstrate that the PM_CMPS methodology reduces the predicted standard deviations to values that are smaller than either the computed or the experimentally measured ones, even for responses (e.g., the outlet water flow rate) for which no measurements are available. These improvements stem from the global characteristics of the PM_CMPS methodology, which combines all of the available information simultaneously in phase-space, as opposed to combining it sequentially, as in current data assimilation procedures.
\end{abstract}

Keywords: adjoint sensitivity analysis; data assimilation; model calibration; best-estimate predictions; reduced predicted uncertainties

\section{Introduction}

In the present work, the predictive modeling of the counter-flow cooling tower presented in [1] is further developed by applying the "predictive modeling for coupled multi-physics systems" (PM_CMPS) methodology recently developed in [2]. The PM_CMPS methodology constructs a prior distribution for the parameters and responses by using all of the available computational and experimental information, and by relying on the maximum entropy principle to maximize the impact of all available information and minimize the impact of ignorance. Subsequently, the PM_CMPS methodology [2] constructs formally the posterior distribution using Bayes' theorem, and then evaluates asymptotically, to first-order sensitivities, the posterior distribution using the saddle-point method to obtain explicit formulas for the predicted optimal nominal values for the model responses and parameters, along with reduced predicted uncertainties (i.e., reduced predicted standard deviations) for the predicted model parameters and responses. The PM_CMPS methodology has been successfully applied to the analysis of large-scale experiments and the experimental validation of reactor design codes of interest to reactor physics [3,4], light water reactors [5] and sodium-cooled fast reactors [6]. 
The PM_CMPS methodology relies fundamentally on the sensitivities to model parameters of the measured model responses, which, in this work, are as follows: (i) the outlet air temperature; (ii) the outlet water temperature; (iii) the outlet water mass flow rate; and (iv) the air outlet relative humidity. The expressions, numerical results, and relative rankings of the sensitivities of these responses are presented in Section 2.1. These sensitivities are subsequently used in Section 2.2 for assimilating experimental data in order to "calibrate" the model parameters, and for obtaining best-estimate predicted results with reduced predicted uncertainties. Section 3 concludes this work by discussing the significance of the results presented herein in the context of ongoing work aimed at further applications and generalization of the adjoint sensitivity analysis and PM_CMPS methodologies.

\section{Results}

It has been shown in the accompanying PART I [1] that the total sensitivity of a model response $R\left(\mathbf{m}_{w}, \mathbf{T}_{w}, \mathbf{T}_{a}, \boldsymbol{\omega} ; \boldsymbol{\alpha}\right)$ to arbitrary variations in the model's parameters $\delta \boldsymbol{\alpha} \equiv\left(\delta \alpha_{1}, \ldots, \delta \alpha_{N_{\alpha}}\right)$ and state functions $\delta \mathbf{m}_{w}, \delta \mathbf{T}_{w}, \delta \mathbf{T}_{a}, \delta \boldsymbol{w}$, around the nominal values $\left(\mathbf{m}_{w}^{0}, \mathbf{T}_{w}^{0}, \mathbf{T}_{a}^{0}, \boldsymbol{\omega}^{0} ; \boldsymbol{\alpha}^{0}\right)$ of the parameters and state functions, is provided by the G-differential of the model's response to these variations. This G-differential was denoted as $D R\left(\mathbf{m}_{w}^{0}, \mathbf{T}_{w}^{0}, \mathbf{T}_{a}^{0}, \boldsymbol{\omega}^{0} ; \boldsymbol{\alpha}^{0} ; \delta \mathbf{m}_{w}, \delta \mathbf{T}_{w}, \delta \mathbf{T}_{a}, \delta \boldsymbol{\omega} ; \delta \boldsymbol{\alpha}\right)$, and was expressed in terms of the adjoint sensitivity functions as follows:

$$
D R\left(\mathbf{m}_{w}^{0}, \mathbf{T}_{w}^{0}, \mathbf{T}_{a}^{0}, \boldsymbol{\omega}^{0} ; \boldsymbol{\alpha}^{0} ; \delta \mathbf{m}_{w}, \delta \mathbf{T}_{w}, \delta \mathbf{T}_{a}, \delta \boldsymbol{\omega} ; \delta \boldsymbol{\alpha}\right)=\sum_{i=1}^{N_{\alpha}}\left(\frac{\partial R}{\partial \alpha_{i}} \delta \alpha_{i}\right)+D R_{\text {indirect }},
$$

where the so-called "indirect effect" term, $D R_{\text {indirect }}$, is given by:

$$
D R_{\text {indirect }} \equiv \boldsymbol{\mu}_{w} \cdot \mathbf{Q}_{1}+\boldsymbol{\tau}_{w} \cdot \mathbf{Q}_{2}+\boldsymbol{\tau}_{a} \cdot \mathbf{Q}_{3}+\mathbf{o} \cdot \mathbf{Q}_{4}
$$

and where the vector $\left[\boldsymbol{\mu}_{w}, \boldsymbol{\tau}_{w}, \boldsymbol{\tau}_{a}, \mathbf{o}\right]^{+}$is the solution of the following adjoint sensitivity system:

$$
\left(\begin{array}{cccc}
\mathbf{A}_{1}^{+} & \mathbf{A}_{2}^{+} & \mathbf{A}_{3}^{+} & \mathbf{A}_{4}^{+} \\
\mathbf{B}_{1}^{+} & \mathbf{B}_{2}^{+} & \mathbf{B}_{3}^{+} & \mathbf{B}_{4}^{+} \\
\mathbf{C}_{1}^{+} & \mathbf{C}_{2}^{+} & \mathbf{C}_{3}^{+} & \mathbf{C}_{4}^{+} \\
\mathbf{D}_{1}^{+} & \mathbf{D}_{2}^{+} & \mathbf{D}_{3}^{+} & \mathbf{D}_{4}^{+}
\end{array}\right)\left(\begin{array}{c}
\boldsymbol{\mu}_{w} \\
\boldsymbol{\tau}_{w} \\
\boldsymbol{\tau}_{a} \\
\mathbf{o}
\end{array}\right)=\left(\begin{array}{c}
\mathbf{R}_{1} \\
\mathbf{R}_{2} \\
\mathbf{R}_{3} \\
\mathbf{R}_{4}
\end{array}\right) .
$$

Furthermore, the sources $\mathbf{R}_{\ell} \equiv\left(r_{\ell}^{(1)}, \ldots, r_{\ell}^{(I)}\right), \ell=1,2,3,4$, for the adjoint sensitivity system represented by Equation (3) are the functional derivatives of the model responses with respect to the state functions, i.e.:

$$
r_{1}^{(i)} \equiv \frac{\partial R}{\partial m_{w}^{(i+1)}} ; r_{2}^{(i)} \equiv \frac{\partial R}{\partial T_{w}^{(i+1)}} ; r_{3}^{(i)} \equiv \frac{\partial R}{\partial T_{a}^{(i)}} ; r_{4}^{(i)} \equiv \frac{\partial R}{\partial \omega^{(i)}} ; \quad i=1, \ldots, I
$$

while the components of the vectors $\mathbf{Q}_{\ell} \equiv\left(q_{\ell}^{(1)}, \ldots, q_{\ell}^{(I)}\right), \ell=1,2,3,4$, in Equation (2) are the derivatives of the model's equations with respect to model parameters, namely:

$$
q_{\ell}^{(i)} \equiv \sum_{j=1}^{N_{\alpha}}\left(\frac{\partial N_{\ell}^{(i)}}{\partial \alpha_{j}} \delta \alpha_{j}\right) ; i=1, \ldots, I ; \ell=1,2,3,4 .
$$

The explicit expressions of the vectors $\mathbf{Q}_{\ell} \equiv\left(q_{\ell}^{(1)}, \ldots, q_{\ell}^{(I)}\right), \ell=1,2,3,4$ are provided in Appendix A. The model responses of interest in this work are the following quantities: (i) the outlet air temperature, $T_{a}^{(1)}$; (ii) the outlet water temperature, $T_{w}^{(50)}$; (iii) the outlet water flow rate, $m_{w}^{(50)}$; and (iv) the outlet air relative humidity, $R H^{(1)}$. Except for the water outlet flow rate $m_{w}^{(50)}$, these responses have been measured experimentally $[7,8]$, and the first four moments of their respective statistical distributions have been quantified in [1]. 


\subsection{Sensitivity Analysis Results and Rankings}

As has been discussed in the accompanying PART I [1], there are a total of 8079 measured benchmark data sets for the cooling tower model with the "fan-on," with a drafted air exit velocity at $10 \mathrm{~m} / \mathrm{s}$ at the shroud. For this velocity (and corresponding air flow rate), the Reynolds number is around 4500, which means that the flow within the cooling tower is in the "transitional flow and heat transfer" regime. As has also been discussed in [1], 7668 benchmark data sets (out of the total of 8079 data sets) are considered to correspond to the "unsaturated conditions" which are analyzed in this work. The nominal values for boundary and atmospheric conditions used in this work were obtained, as described in [1], from the statistics of these 7668 benchmark data sets corresponding to "unsaturated conditions." In turn, these "unsaturated" boundary and atmospheric conditions were used to obtain the sensitivity results reported, below, in this Subsection. Sub-subsections 2.1.1 through 2.1.4, below, provide the numerical values and rankings, in descending order, of the relative sensitivities computed using the adjoint sensitivity analysis methodology for the four model responses $T_{a}^{(1)}, T_{w}^{(50)}$, $m_{w}^{(50)}$ and $R H^{(1)}$. Note that the relative sensitivity, $R S\left(\alpha_{i}\right)$, of a response $R\left(\alpha_{i}\right)$ to a parameter $\alpha_{i}$ is defined as $R S\left(\alpha_{i}\right) \equiv\left[d R\left(\alpha_{i}\right) / d \alpha_{i}\right]\left[\alpha_{i} / R\left(\alpha_{i}\right)\right]$. Thus, the relative sensitivities are unit-less and are very useful in ranking the sensitivities to highlight their relative importance for the respective response. Thus, a relative sensitivity of 1.00 indicates that a change of $1 \%$ in the respective parameter will induce a $1 \%$ change in a response that is linear in the respective sensitivity. The higher the relative sensitivity, the more important the respective parameter to the respective response.

\subsubsection{Relative Sensitivities of the Outlet Air Temperature, $T_{a}^{(1)}$}

The sensitivities of the air outlet temperature with respect to all of the model's parameters have been computed using Equations (1) and (2). The numerical results and ranking of the relative sensitivities, in descending order of their magnitudes, are provided in Table 1 below, along with their respective relative standard deviations.

Table 1. Ranked relative sensitivities of the outlet air temperature $T_{a}^{(1)}$.

\begin{tabular}{|c|c|c|c|c|}
\hline Rank \# & Parameter $\left(\alpha_{i}\right)$ & Nominal Value & $\begin{array}{c}\text { Relative Sensitivity } \\
\qquad S S\left(\alpha_{i}\right)\end{array}$ & $\begin{array}{c}\text { Relative Standard } \\
\text { Deviation (\%) }\end{array}$ \\
\hline 1 & Inlet air temperature, $T_{a, i n}$ & $299.11 \mathrm{~K}$ & 0.4858 & 1.39 \\
\hline 2 & Air temperature (dry bulb), $T_{d b}$ & $299.11 \mathrm{~K}$ & 0.4829 & 1.39 \\
\hline 4 & Dew point temperature, $T_{d p}$ & $292.05 \mathrm{~K}$ & 0.1834 & 0.81 \\
\hline 5 & $\mathrm{P}_{\mathrm{vs}}(\mathrm{T})$ parameter, $a_{0}$ & 25.5943 & -0.0945 & 0.04 \\
\hline 6 & $\mathrm{P}_{\mathrm{vs}}(\mathrm{T})$ parameter, $a_{1}$ & -5229.89 & 0.0618 & 0.08 \\
\hline 7 & Inlet air humidity ratio, $\omega_{\text {in }}$ & 0.0138 & 0.0100 & 14.93 \\
\hline 10 & Wetted fraction of fill surface area, $w_{t s a}$ & 1.0 & -0.0049 & 0.00 \\
\hline 11 & Nusselt number, $\mathrm{Nu}$ & 14.94 & -0.0049 & 34.0 \\
\hline 12 & Fill section surface area, $A_{\text {surf }}$ & $14221 \mathrm{~m}^{2}$ & -0.0049 & 25.0 \\
\hline 13 & Dynamic viscosity of air at $\mathrm{T}=300 \mathrm{~K}, \mu$ & $1.983 \times 10^{-5} \mathrm{~kg} /(\mathrm{m} \cdot \mathrm{s})$ & 0.0045 & 4.88 \\
\hline 14 & $\mathrm{Nu}$ parameter, $a_{1, N u}$ & 0.0031498 & -0.0045 & 31.75 \\
\hline 15 & Reynolds number, $R e_{d}$ & 4428 & -0.0045 & 15.17 \\
\hline 16 & Fill section flow area, $A_{\text {fill }}$ & $67.29 \mathrm{~m}^{2}$ & 0.0045 & 10.0 \\
\hline 22 & Inlet air mass flow rate, $m_{a}$ & $155.07 \mathrm{~kg} / \mathrm{s}$ & -0.0028 & 10.26 \\
\hline 23 & Heat transfer coefficient multiplier, $f_{h t}$ & $1.0^{\circ}$ & -0.0026 & 50.0 \\
\hline 24 & Thermal conductivity of air at $\mathrm{T}=300 \mathrm{~K}, k_{\text {air }}$ & $0.02624 \mathrm{~W} /(\mathrm{m} \cdot \mathrm{K})$ & -0.0026 & 6.04 \\
\hline 25 & Mass transfer coefficient multiplier, $f_{m t}$ & 1.0 & -0.0022 & 50.0 \\
\hline 26 & Sherwood number, $S h$ & 14.13 & -0.0022 & 34.25 \\
\hline 27 & $\mathrm{D}_{\mathrm{av}}(\mathrm{T})$ parameter, $a_{2, d a v}$ & $-6.1681 \times 10^{-3}$ & -0.0019 & 0.37 \\
\hline 28 & $\mathrm{~h}_{\mathrm{f}}(\mathrm{T})$ parameter, $a_{0 f}$ & $-1,143,423$ & -0.0017 & 0.05 \\
\hline 29 & $\mathrm{D}_{\mathrm{av}}(\mathrm{T})$ parameter, $a_{0, d a v}$ & $7.06085 \times 10^{-9}$ & -0.0015 & 0 \\
\hline 30 & Atmospheric pressure, $P_{a t m}$ & $100,586 \mathrm{~Pa}$ & -0.0013 & 0.40 \\
\hline 31 & Kinematic viscosity of air at $300 \mathrm{~K}, v$ & $1.568 \times 10^{-5} \mathrm{~m}^{2} / \mathrm{s}$ & -0.00074 & 12.09 \\
\hline 32 & Prandlt number of air at $\mathrm{T}=80 \mathrm{C}, \mathrm{Pr}$ & 0.708 & 0.00074 & 0.71 \\
\hline 33 & Schmidt number, $S c$ & 0.60 & -0.00074 & 12.41 \\
\hline 34 & $\mathrm{~h}_{\mathrm{g}}(\mathrm{T})$ parameter, $a_{1 g}$ & 1815.437 & -0.00074 & 0.19 \\
\hline
\end{tabular}


Table 1. Cont.

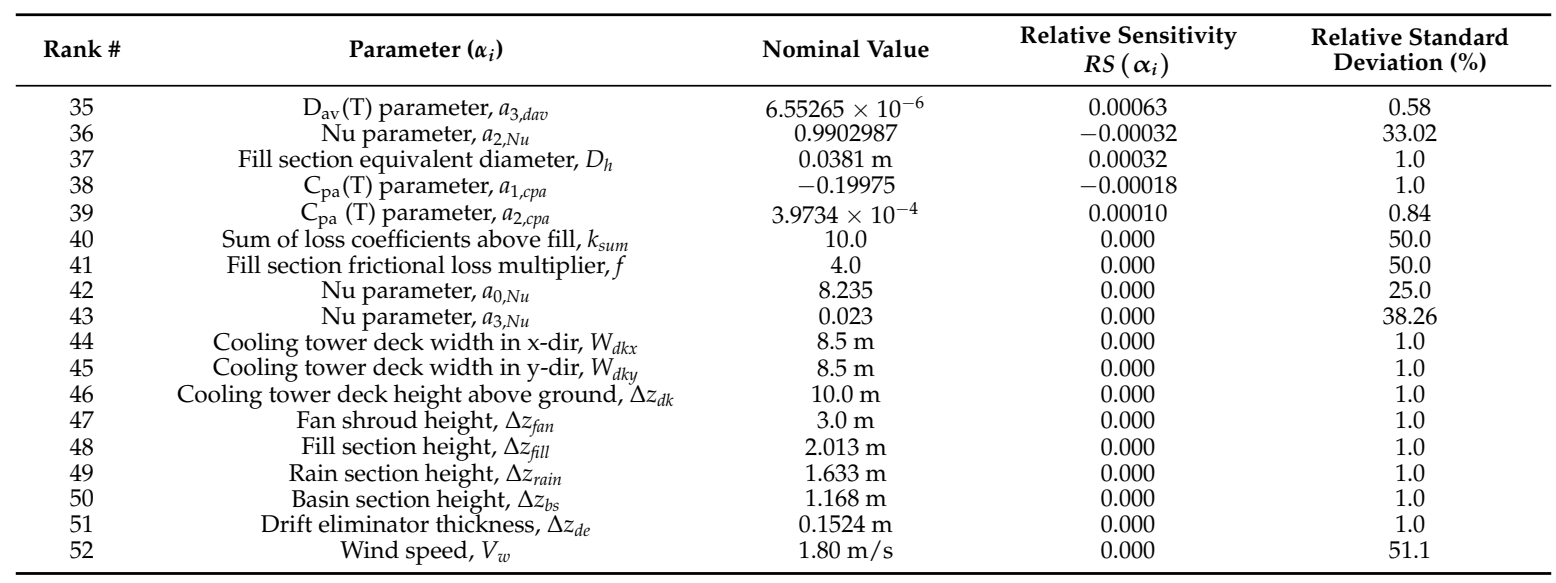

As the results in Table 1 indicate, the first five parameters (i.e., $T_{a, i n}, T_{d b}, T_{w, i n}, T_{d p}, a_{0}$ ) have relative sensitivities between ca. 10\% and 50\%, and are therefore the most important for the air outlet temperature response, $T_{a}^{(1)}$. The two largest sensitivities have values of $48 \%$, which means that a $1 \%$ change in $T_{a, i n}$ or $T_{d b}$ would induce a $0.48 \%$ change in $T_{a}^{(1)}$. The next two parameters (i.e., $a_{1}$ and $\omega_{i n}$ ) have relative sensitivities between $1 \%$ and $6 \%$, and are therefore somewhat important. Parameters \#8 through \#16 (i.e., $D_{\text {fan }}, a_{1 f}, w_{t s a}, N u, A_{\text {surf }}, \mu, a_{1, N u}, \operatorname{Re}_{d}, A_{\text {fill }}$ ) have relative sensitivities of the order of $0.5 \%$. The remaining 36 parameters are relatively unimportant for this response, having relative sensitivities smaller than $1 \%$ of the largest relative sensitivity (with respect to $T_{a, i n}$ ) for this response. Positive sensitivities imply that a positive change in the respective parameter would cause an increase in the response, while negative sensitivities imply that a positive change in the respective parameter would cause a decrease in the response.

\subsubsection{Relative Sensitivities of the Outlet Water Temperature, $T_{w}^{(50)}$}

The results and ranking of the relative sensitivities of the outlet water temperature with respect to the most important 12 parameters for this response are listed in Table 2.

Table 2. Most important relative sensitivities of the outlet water temperature, $T_{w}^{(50)}$.

\begin{tabular}{|c|c|c|c|c|}
\hline Rank \# & Parameter $\left(\alpha_{i}\right)$ & Nominal Value & $\begin{array}{c}\text { Relative Sensitivity } \\
R S\left(\alpha_{i}\right)\end{array}$ & $\begin{array}{c}\text { Relative Standard } \\
\text { Deviation (\%) }\end{array}$ \\
\hline 1 & Dew point temperature, $T_{d p}$ & $292.05 \mathrm{~K}$ & 0.5482 & 0.81 \\
\hline 2 & Inlet air temperature, $T$ & $299.11 \mathrm{~K}$ & 0.2318 & 1.39 \\
\hline 4 & $\mathrm{P}_{\mathrm{vs}}(\mathrm{T})$ parameters, $a_{0}$ & 25.5943 & -0.1949 & 0.04 \\
\hline 5 & $\mathrm{P}_{\mathrm{vs}}(\mathrm{T})$ parameters, $a_{1}$ & -5229.89 & 0.1282 & 0.08 \\
\hline 6 & Inlet water temperature, $T_{w, i n}$ & $298.79 \mathrm{~K}$ & 0.1066 & 0.57 \\
\hline 7 & Inlet air humidity ratio, $\omega_{i n}$ & 0.0138 & 0.0299 & 14.93 \\
\hline 10 & $\mathrm{D}_{\mathrm{av}}\left(\mathrm{T}_{\mathrm{db}}\right)$ parameter, $a_{1, \text { dav }}$ & 2.653 & 0.0071 & 0.11 \\
\hline 11 & Enthalpy $h_{g}(\mathrm{~T})$ parameter, $a_{0 g}$ & $2,005,744$ & -0.0062 & 0.05 \\
\hline 12 & Sherwood number, $S h$ & 14.13 & -0.0056 & 34.25 \\
\hline
\end{tabular}

The largest sensitivity of $T_{w}^{(50)}$ is to the parameter $T_{d p}$, and has the value of 0.548 ; this means that a $1 \%$ increase in $T_{d b}$ would induce a $0.548 \%$ increase in $T_{w}^{(50)}$. The sensitivities to the remaining 40 model parameters have not been listed since they are smaller than $1 \%$ of the largest sensitivity (with respect to $T_{d p}$ ) for this response.

\subsubsection{Relative Sensitivities of the Outlet Water Mass Flow Rate, $m_{w}^{(50)}$}

The results and ranking of the relative sensitivities of the outlet water mass flow rate with respect to the most important 10 parameters for this response are listed in Table 3. This response is most 
sensitive to $m_{w, \text { in }}$ (a $1 \%$ increase in this parameter would cause a $1.01 \%$ increase in the response) and the second largest sensitivity is to the parameter $T_{w, \text { in }}$ (a $1 \%$ increase in this parameter would cause a $0.447 \%$ decrease in the response). The sensitivities to the remaining 42 model parameters have not been listed since they are smaller than $1 \%$ of the largest sensitivity (with respect to $m_{w, i n}$ ) for this response.

Table 3. Most important relative sensitivities of the outlet water mass flow rate, $m_{w}^{(50)}$.

\begin{tabular}{|c|c|c|c|c|}
\hline Rank \# & Parameter $\left(\alpha_{i}\right)$ & Nominal Value & $\begin{array}{c}\text { Relative Sensitivity } \\
\operatorname{RS}\left(\alpha_{i}\right)\end{array}$ & $\begin{array}{c}\text { Relative Standard } \\
\text { Deviation (\%) }\end{array}$ \\
\hline 1 & Inlet water mass flow rate, $m_{w \text { in }}$ & $44.02 \mathrm{~kg} / \mathrm{s}$ & 1.0060 & 5.00 \\
\hline 2 & Inlet water temperature, $T_{w, i n}$ & $298.79 \mathrm{~K}$ & -0.4474 & 0.57 \\
\hline 4 & Pvs(T) parameters, $a_{0}$ & 25.5943 & -0.1416 & 0.04 \\
\hline 5 & Air temperature (dry bulb), $T_{d b}$ & $299.11 \mathrm{~K}$ & -0.1184 & 1.39 \\
\hline 6 & Inlet air temperature, $T_{a, i n}$ & $299.11 \mathrm{~K}$ & -0.1134 & 1.39 \\
\hline 7 & Pvs(T) parameters, $a_{1}$ & -5229.89 & 0.0930 & 0.08 \\
\hline 10 & Inlet air mass flow rate, $m_{a}$ & $155.07 \mathrm{~kg} / \mathrm{s}$ & -0.0058 & 10.26 \\
\hline
\end{tabular}

2.1.4. Relative Sensitivities of the Outlet Air Relative Humidity, $R H^{(1)}$

The results and ranking of the relative sensitivities of the outlet air relative humidity with respect to the most important 20 parameters for this response are listed in Table 4 . The first three sensitivities of this response are quite large (relative sensitivities larger than unity are customarily considered to be very significant). In particular, an increase of $1 \%$ in $T_{a, i n}$ or $T_{d b}$ would cause a decrease in the response of $6.66 \%$ or $6.525 \%$, respectively. On the other hand, an increase of $1 \%$ in $T_{d p}$ would cause an increase of $5.75 \%$ in the response. The sensitivities to the remaining 32 model parameters have not been listed since they are smaller than $1 \%$ of the largest sensitivity (with respect to $T_{a, i n}$ ) for this response.

Table 4. Most important relative sensitivities of the outlet air relative humidity, $R H^{(1)}$.

\begin{tabular}{|c|c|c|c|c|}
\hline Rank \# & Parameter $\left(\alpha_{i}\right)$ & Nominal Value & $\begin{array}{c}\text { Relative Sensitivity } \\
\text { RS }\left(\alpha_{i}\right)\end{array}$ & $\begin{array}{c}\text { Relative Standard } \\
\text { Deviation (\%) }\end{array}$ \\
\hline 1 & Inlet air temperature, $T_{a, i n}$ & $299.11 \mathrm{~K}$ & -6.660 & 1.39 \\
\hline 2 & Air temperature (dry bulb), $T_{d b}$ & $299.11 \mathrm{~K}$ & -6.525 & 1.39 \\
\hline 4 & Inlet water temperature, $T_{w, i n}$ & $298.79 \mathrm{~K}$ & 0.747 & 0.57 \\
\hline 5 & Inlet air humidity ratio, $\omega_{i n}$ & 0.0138 & 0.3141 & 14.93 \\
\hline 6 & $\mathrm{P}_{\mathrm{vs}}(\mathrm{T})$ parameters, $a_{0}$ & 25.5943 & -0.3123 & 0.04 \\
\hline 7 & Wetted fraction of fill surface area, $w_{t s a}$ & 1.0 & 0.1487 & 0.00 \\
\hline 10 & Dynamic viscosity of air at $\mathrm{T}=300 \mathrm{~K}, \mu$ & $1.983 \times 10^{-5} \mathrm{~kg} /(\mathrm{m} \cdot \mathrm{s})$ & -0.1388 & 4.88 \\
\hline 11 & Nu parameters, $a_{1, N u}$ & 0.0031498 & 0.1388 & 31.75 \\
\hline 12 & Fill section flow area, $A_{\text {fill }}$ & $67.29 \mathrm{~m}^{2}$ & -0.1388 & 10.0 \\
\hline 13 & Reynold's number, $R e$ & 4428 & 0.1388 & 15.17 \\
\hline 14 & $\mathrm{D}_{\mathrm{av}}\left(\mathrm{T}_{\mathrm{db}}\right)$ parameter, $a_{1, d a v}$ & 2.65322 & -0.1297 & 0.11 \\
\hline 15 & Mass transfer coefficient multiplier, $f_{m t}$ & 1.0 & 0.1023 & 50.0 \\
\hline 16 & Sherwood number, $\mathrm{Sh}$ & 14.13 & 0.1023 & 34.25 \\
\hline
\end{tabular}

Overall, the outlet air relative humidity, $R H^{(1)}$, displays the largest sensitivities, so this response is the most sensitive to parameter variations. The other responses, namely the outlet air temperature, the outlet water temperature, and the outlet water mass flow rate display sensitivities of comparable magnitudes.

\subsection{Experimental Data Assimilation, Model Calibration and Best-Estimate Predicted Results with Reduced Predicted Uncertainties}

This subsection presents the results of applying the Predictive Modeling of Coupled Multi-Physics Systems (PM_CMPS) methodology [2] to the counter-flow cooling tower model. The PM_CMPS methodology [2] encompasses into a unified conceptual and mathematical framework, the concepts of both "forward" and "inverse" modeling, including data assimilation, model calibration and prediction 
of best-estimate values for model parameters and responses, with reduced predicted uncertainties. For the simplest case of a single computational model, such as the counter-flow cooling tower model analyzed in this work, the PM_CMPS methodology considers the following a priori information:

1. A model comprising $N_{\alpha}$ imprecisely known system (model) parameters, $\alpha_{n}$, considered as the components of a (column) vector, $\alpha$, defined as:

$$
\alpha=\left\{\alpha_{n} \mid n=1, \ldots, N_{\alpha}\right\}
$$

The mean values of the model parameters $\alpha_{n}$ are denoted as $\alpha_{n}^{0} \equiv\left\langle\alpha_{n}\right\rangle$, and the covariances between two parameters $\alpha_{i}$ and $\alpha_{j}$ are denoted as $\operatorname{cov}\left(\alpha_{i}, \alpha_{j}\right)$. The mean values $\alpha_{n}^{0}$ are considered to be known a priori, so that the vector $\alpha^{0}$, defined as $\alpha^{0}=\left\{\alpha_{n}^{0} \mid n=1, \ldots, N_{\alpha}\right\}$ is considered to be known a priori. The covariances $\operatorname{cov}\left(\alpha_{i}, \alpha_{j}\right)$ are also considered to be a priori known; these covariances are considered to be the elements of the a priori known parameter covariance matrix, denoted as $\mathbf{C}_{\alpha \alpha}^{\left(N_{\alpha} \times N_{\alpha}\right)}$ and defined as:

$$
\mathbf{C}_{\alpha \alpha}^{\left(N_{\alpha} \times N_{\alpha}\right)} \equiv\left[\operatorname{cov}\left(\alpha_{i}, \alpha_{j}\right)\right]_{N_{\alpha} \times N_{\alpha}} \equiv\left\langle\left(\alpha_{i}-\alpha_{i}^{0}\right)\left(\alpha_{j}-\alpha_{j}^{0}\right)\right\rangle_{N_{\alpha} \times N_{\alpha}} ; i, j=1, \ldots, N_{\alpha}
$$

2. Also associated with the model are $N_{r}$ experimentally measured responses, $r_{i}$, considered to be components of the column vector:

$$
\mathbf{r}=\left\{r_{i} \mid i=1, \ldots, N_{r}\right\}
$$

The mean values, denoted as $r_{i}^{m}$, of the measured responses, $r_{i}$, and the covariances, denoted as $\left\langle\left(r_{i}-r_{i}^{m}\right)\left(r_{j}-r_{j}^{m}\right)\right\rangle$, between two measured responses, $r_{i}$ and $r_{j}$, are also considered to be known a priori. The mean measured values $r_{i}^{m}$ will be considered to constitute the components of the vector $\mathbf{r}^{m}$ defined as:

$$
\mathbf{r}^{m}=\left\{r_{i}^{m} \mid i=1, \ldots, N_{r}\right\}, \quad r_{i}^{m} \equiv\left\langle r_{i}\right\rangle, i=1, \ldots, N_{r},
$$

and the covariances $\left\langle\left(r_{i}-r_{i}^{m}\right)\left(r_{j}-r_{j}^{m}\right)\right\rangle$ of the measured responses are considered to be components of the a priori known measured covariance matrix, denoted as $\mathbf{C}_{r r}^{\left(N_{r} \times N_{r}\right)}$, and defined as:

$$
\mathbf{C}_{r r}^{\left(N_{r} \times N_{r}\right)} \equiv\left\langle\left(r_{i}-r_{i}^{m}\right)\left(r_{j}-r_{j}^{m}\right)\right\rangle_{N_{r} \times N_{r}}, i, j=1, \ldots, N_{r} .
$$

3. In the most general case, correlations may also exist among all parameters and responses. Such correlations are quantified through a priori known parameter-response matrices, denoted as $\mathbf{C}_{\alpha r}^{\left(N_{\alpha} \times N_{r}\right)}$, and defined as follows:

$$
\mathbf{C}_{\alpha r}^{\left(N_{\alpha} \times N_{r}\right)} \equiv\left\langle\left(\boldsymbol{\alpha}-\boldsymbol{\alpha}^{0}\right)\left(\mathbf{r}-\mathbf{r}^{m}\right)^{+}\right\rangle=\left[\mathbf{C}_{r \alpha}^{\left(N_{r} \times N_{\alpha}\right)}\right]^{+}
$$

To keep the notation simple, the dimensions of the various vectors and matrices will not be shown in subsequent formulas. For a single multi-physics system, as is the case of the cooling tower model under consideration in this work, the quantities predicted by the PM_CMPS methodology [2] are as follows:

A. Optimally predicted "best-estimate" nominal values, $\alpha^{\text {pred }}$, for the model parameters:

$$
\boldsymbol{\alpha}^{\text {pred }}=\boldsymbol{\alpha}^{0}-\left(\mathbf{C}_{\alpha \alpha} \mathbf{S}_{r \alpha}^{+}-\mathbf{C}_{\alpha r}\right)\left[\mathbf{D}_{r r}\right]^{-1}\left[\mathbf{r}^{c}\left(\boldsymbol{\alpha}^{0}, \boldsymbol{\beta}^{0}\right)-\mathbf{r}^{m}\right],
$$

where the matrix $\mathbf{D}_{r r}$ is defined as:

$$
\mathbf{D}_{r r}=\mathbf{S}_{r \alpha} \mathbf{C}_{\alpha \alpha} \mathbf{S}_{r \alpha}^{+}-\mathbf{S}_{r \alpha} \mathbf{C}_{\alpha r}-\mathbf{C}_{\alpha r}^{+} \mathbf{S}_{r \alpha}^{+}+\mathbf{C}_{r r}
$$


and the components of the matrix $\mathbf{S}_{r \alpha}^{\left(N_{r} \times N_{\alpha}\right)}$ are the first-order sensitivities (i.e., functional derivatives) of all responses with respect to all model parameters, defined as follows:

$$
\mathbf{S}_{r \alpha}^{N_{r} \times N_{\alpha}} \equiv\left(\begin{array}{ccc}
\frac{\partial r_{1}}{\partial \alpha_{1}} & \cdots & \frac{\partial r_{1}}{\partial \alpha_{N_{\alpha}}} \\
\vdots & \ddots & \vdots \\
\frac{\partial r_{N_{r}}}{\partial \alpha_{1}} & \cdots & \frac{\partial r_{N_{r}}}{\partial \alpha_{N_{\alpha}}}
\end{array}\right) .
$$

It is important to note that the first term on the right side of Equation (13) is the covariance matrix of the computed responses, $\mathbf{C}_{r r}^{c o m p}$, when only the first-order sensitivities are taken into account, i.e.:

$$
\mathbf{C}_{r r}^{c o m p}=\mathbf{S}_{r \alpha} \mathbf{C}_{\alpha \alpha} \mathbf{S}_{r \alpha}^{+} .
$$

B. Reduced predicted uncertainties, $\mathbf{C}_{\alpha \alpha}^{\text {pred }}$, for the predicted nominal parameter values, given by the expression below:

$$
\mathbf{C}_{\alpha \alpha}^{p r e d}=\mathbf{C}_{\alpha \alpha}-\left(\mathbf{C}_{\alpha \alpha} \mathbf{S}_{r \alpha}^{+}-\mathbf{C}_{\alpha r}\right)\left[\mathbf{D}_{r r}\right]^{-1}\left(\mathbf{C}_{\alpha \alpha} \mathbf{S}_{r \alpha}^{+}-\mathbf{C}_{\alpha r}\right)^{+} ;
$$

C. Optimally predicted "best-estimate" nominal values, $\mathbf{r}$ pred , for the model responses, given by the expression below:

$$
\mathbf{r}^{\text {pred }}=\mathbf{r}^{m}-\left(\mathbf{C}_{\alpha r}^{+} \mathbf{S}_{r \alpha}^{+}-\mathbf{C}_{r r}\right)\left[\mathbf{D}_{r r}\right]^{-1}\left[\mathbf{r}^{c}\left(\boldsymbol{\alpha}^{0}, \boldsymbol{\beta}^{0}\right)-\mathbf{r}^{m}\right]
$$

D. Reduced predicted uncertainties, $\mathbf{C}_{r r}^{\text {pred }}$, for the predicted nominal response values, given by the expression below:

$$
\mathbf{C}_{r r}^{\text {pred }}=\mathbf{C}_{r r}-\left(\mathbf{C}_{\alpha r}^{+} \mathbf{S}_{r \alpha}^{+}-\mathbf{C}_{r r}\right)\left[\mathbf{D}_{r r}\right]^{-1}\left(\mathbf{C}_{\alpha r}^{+} \mathbf{S}_{r \alpha}^{+}-\mathbf{C}_{r r}\right)^{+}
$$

E. Predicted correlations, $\mathbf{C}_{\alpha r}^{p r e d}$, between the predicted model parameters and responses, given by the expression below:

$$
\mathbf{C}_{\alpha r}^{p r e d}=\mathbf{C}_{\alpha r}-\left(\mathbf{C}_{\alpha \alpha} \mathbf{S}_{r \alpha}^{+}-\mathbf{C}_{\alpha r}\right)\left[\mathbf{D}_{r r}\right]^{-1}\left(\mathbf{C}_{\alpha r}^{+} \mathbf{S}_{r \alpha}^{+}-\mathbf{C}_{r r}\right)^{+}
$$

The expressions given in Equations (6) through (19) can also be obtained from the results presented originally in [9] for the particular case of a time-independent single multi-physics system. Note that if the model is perfect (which means that $\mathbf{C}_{\alpha \alpha}=0$ and $\mathbf{C}_{\alpha r}=0$ ), Equations (6) through (19) would yield $\boldsymbol{\alpha}^{\text {pred }}=\boldsymbol{\alpha}^{0}$ and $\mathbf{r}^{\text {pred }}=\mathbf{r}^{c}\left(\boldsymbol{\alpha}^{0}, \boldsymbol{\beta}^{0}\right)$, without any accompanying uncertainties (i.e., $\mathbf{C}_{r r}^{\text {pred }}=0, \mathbf{C}_{\alpha \alpha}^{\text {pred }}=0$, $\mathbf{C}_{\alpha r}^{\text {pred }}=0$ ). In other words, for a perfect model, the PM_CMPS methodology predicts values for the responses and the parameters that would coincide with the model's original corresponding parameter and computed responses (assumed to be perfect), and the experimental measurements would have no effect on the predictions (as would be expected, since imperfect measurements could not possibly improve a "perfect" model's predictions). On the other hand, if the measurements were perfect, (i.e., $\mathbf{C}_{r r}=0$ and $\mathbf{C}_{\alpha r}=0$ ), but the model were imperfect, then Equations (6) through (19) would yield $\boldsymbol{\alpha}^{\text {pred }}=\alpha^{0}-\mathbf{C}_{\alpha \alpha} S_{r \alpha}^{+}\left[\mathbf{S}_{r \alpha} \mathbf{C}_{\alpha \alpha} \mathbf{S}_{r \alpha}^{+}\right]^{-1} \mathbf{r}^{d}\left(\boldsymbol{\alpha}^{0}\right), \mathbf{C}_{\alpha \alpha}^{p r e d}=\mathbf{C}_{\alpha \alpha}-\mathbf{C}_{\alpha \alpha} \mathbf{S}_{r \alpha}^{+}\left[\mathbf{S}_{r \alpha} \mathbf{C}_{\alpha \alpha} \mathbf{S}_{r \alpha}^{+}\right]^{-1} \mathbf{S}_{r \alpha} \mathbf{C}_{\alpha \alpha}$, $\mathbf{r}^{\text {pred }}=\mathbf{r}^{m}, \mathbf{C}_{r r}^{\text {pred }}=0, \mathbf{C}_{\alpha r}^{\text {pred }}=0$. In other words, in the case of perfect measurements, the PM_CMPS predicted values for the responses would coincide with the measured values (assumed to be perfect), while the model's uncertain parameters would be calibrated by taking the respective measurements into account to yield improved nominal values and reduced parameters uncertainties.

The a priori response-parameter covariance matrix, $\mathbf{C}_{r \alpha}$, has been already computed in [1], Equation (A5), and is reproduced below: 


$$
\operatorname{Cov}\left(T_{a, \text { out }}^{\text {meas }}, T_{w, \text { out }}^{\text {meas }}, R H^{\text {meas }}, \alpha_{1}, \ldots, \alpha_{52}\right) \triangleq \mathbf{C}_{r \alpha}=\left(\begin{array}{ccccccc}
12.96 & 3.51 & 2.33 & -447.09 & 0 & \cdots & 0 \\
3.35 & 3.05 & 1.89 & -93.58 & 0 & \cdots & 0 \\
-54.16 & 1.73 & -2.27 & 1831.03 & 0 & \cdots & 0
\end{array}\right) .
$$

where the measured correlated parameters are: $\alpha_{1} \equiv \mathrm{T}_{d b}, \alpha_{2} \equiv \mathrm{T}_{d p}, \alpha_{3} \equiv \mathrm{T}_{w, \text { in }}$, and $\alpha_{4} \equiv P_{a t m}$.

The a priori parameter covariance matrix, $\mathbf{C}_{\alpha \alpha}$, has also been already computed in [1], Equation (B1) (see the Appendix of PART I.), and is also reproduced below:

$$
\begin{aligned}
\mathbf{C}_{\alpha \alpha} \triangleq & \left(\begin{array}{cccccc}
\operatorname{Var}\left(\alpha_{1}\right) & \operatorname{Cov}\left(\alpha_{1}, \alpha_{2}\right) & \bullet & \operatorname{Cov}\left(\alpha_{1}, \alpha_{52}\right) \\
\operatorname{Cov}\left(\alpha_{2}, \alpha_{1}\right) & \operatorname{Var}\left(\alpha_{2}\right) & \bullet & \operatorname{Cov}\left(\alpha_{2}, \alpha_{52}\right) \\
\bullet & & \bullet & \bullet & \bullet \\
\operatorname{Cov}\left(\alpha_{52}, \alpha_{1}\right) & \bullet & \bullet & \operatorname{Var}\left(\alpha_{52}\right)
\end{array}\right) \\
& =\left(\begin{array}{ccccccc}
17.37 & 2.83 & 1.81 & -529.26 & 0 & \bullet & 0 \\
2.83 & 5.56 & 2.31 & -87.16 & 0 & \bullet & 0 \\
1.81 & 2.31 & 2.90 & -47.22 & 0 & \bullet & 0 \\
-529.26 & -87.16 & -47.22 & 160597.01 & 0 & \bullet & 0 \\
0 & 0 & 0 & 0 & 0 & \bullet & 0 \\
\bullet & \bullet & \bullet & \bullet & \bullet & \bullet & \bullet \\
0 & 0 & 0 & 0 & 0 & \bullet & 25.81
\end{array}\right)
\end{aligned}
$$

The a priori covariance matrix of the computed responses, $\mathbf{C}_{r r}^{c o m p}$, is obtained by using Equations (15) and (21) together with the sensitivity results presented in Tables 1-4; the final result is given below:

$$
\begin{aligned}
& \mathbf{C}_{r r}^{c o m p} \equiv \operatorname{Cov}\left(T_{a}^{(1)}, T_{w}^{(50)}, R H^{(1)}\right)=\mathbf{S}_{r \alpha} \mathbf{C}_{\alpha \alpha} \mathbf{S}_{r \alpha}^{+} \\
& =\left(\begin{array}{c}
\frac{\partial T_{\alpha}^{(1)}}{\partial \alpha_{1}}, \ldots, \frac{\partial T_{\alpha}^{(1)}}{\partial \alpha_{N \alpha}} \\
\frac{\partial T_{w v}^{(50)}}{\partial \alpha_{1}}, \ldots, \frac{\partial T_{w}^{(50)}}{\partial \alpha_{N \alpha}} \\
\frac{\partial R H^{(1)}}{\partial \alpha_{1}}, \ldots, \frac{\partial R H^{(1)}}{\partial \alpha_{N \alpha}}
\end{array}\right)\left(\begin{array}{cccc}
\operatorname{Var}\left(\alpha_{1}\right) & \operatorname{Cov}\left(\alpha_{1}, \alpha_{2}\right) & \bullet & \operatorname{Cov}\left(\alpha_{1}, \alpha_{52}\right) \\
\operatorname{Cov}\left(\alpha_{2}, \alpha_{1}\right) & \operatorname{Var}\left(\alpha_{2}\right) & \bullet & \operatorname{Cov}\left(\alpha_{2}, \alpha_{52}\right) \\
\bullet & \bullet & \bullet & \bullet \\
\operatorname{Cov}\left(\alpha_{52}, \alpha_{1}\right) & \bullet & \bullet & \operatorname{Var}\left(\alpha_{52}\right)
\end{array}\right)\left(\begin{array}{c}
\frac{\partial T_{a}^{(1)}}{\partial \alpha_{1}}, \ldots, \frac{\partial T_{\alpha}^{(1)}}{\partial N_{\alpha}} \\
\frac{\partial T_{w}^{(50)}}{\partial \alpha_{1}}, \ldots, \frac{\partial T_{w}^{(50)}}{\partial \alpha_{N \alpha}} \\
\frac{\partial R H^{(1)}}{\partial \alpha_{1}}, \ldots, \frac{\partial R H^{(1)}}{\partial \alpha_{N \alpha}}
\end{array}\right)^{+} \\
& =\left(\begin{array}{ccc}
10.87 & 7.19 & -34.81 \\
7.19 & 7.72 & -13.97 \\
-34.81 & -13.97 & 221.88
\end{array}\right) .
\end{aligned}
$$

The a priori covariance matrix, $\operatorname{Cov}\left(T_{a, \text { out }}^{\text {meas }}, T_{w, \text { out }}^{\text {meas }}, R H_{\text {out }}^{\text {meas }}\right) \equiv \mathbf{C}_{r r}$, of the measured responses (namely: the outlet air temperature, $T_{a, \text { out }}^{\text {meas }} \equiv\left[T_{a}^{(1)}\right]^{\text {measured }}$; the outlet water temperature, $T_{w, \text { out }}^{\text {meas }} \equiv\left[T_{w}^{(50)}\right]^{\text {measured }}$, and the outlet air relative humidity, $R H_{\text {out }}^{\text {meas }} \equiv\left[R H^{(1)}\right]^{\text {measured }}$ was also computed in [1], Equation (A4), and is reproduced below:

$$
\operatorname{Cov}\left(T_{a, \text { out }}^{\text {meas }}, T_{w, \text { out }}^{\text {meas }}, R H_{\text {out }}^{\text {meas }}\right) \triangleq \mathbf{C}_{r r}=\left(\begin{array}{ccc}
11.29 & 3.55 & -43.85 \\
3.55 & 2.53 & -5.31 \\
-43.85 & -5.31 & 252.49
\end{array}\right) .
$$

2.2.1. Model Calibration: Predicted Best-Estimated Parameter Values with Reduced Predicted Standard Deviations

The best-estimate nominal parameter values have been computed using Equation (12) in conjunction with the a priori matrices given in Equations (20)-(23) and the sensitivities presented in Tables 1-4. The resulting best-estimate nominal values are listed in Table 5, below. The corresponding best-estimate absolute standard deviations for these parameters are also presented in this table. These 
values are the square-roots of the diagonal elements of the matrix $\mathbf{C}_{\alpha \alpha}^{\text {pred }}$, which is computed using Equation (16) in conjunction with the a priori matrices given in Equations (20)-(23) and the sensitivities presented in Tables 1-4. For comparison, the original nominal parameter values and original absolute standard deviations are also listed. As the results in Table 5 indicate, the predicted best-estimate standard deviations are all smaller or at most equal to (i.e., left unaffected) the original standard deviations. The parameters are affected proportionally to the magnitudes of their corresponding sensitivities: the parameters experiencing the largest reductions in their predicted standard deviations are those having the largest sensitivities.

Table 5. Best-estimated nominal parameter values and their standard deviations.

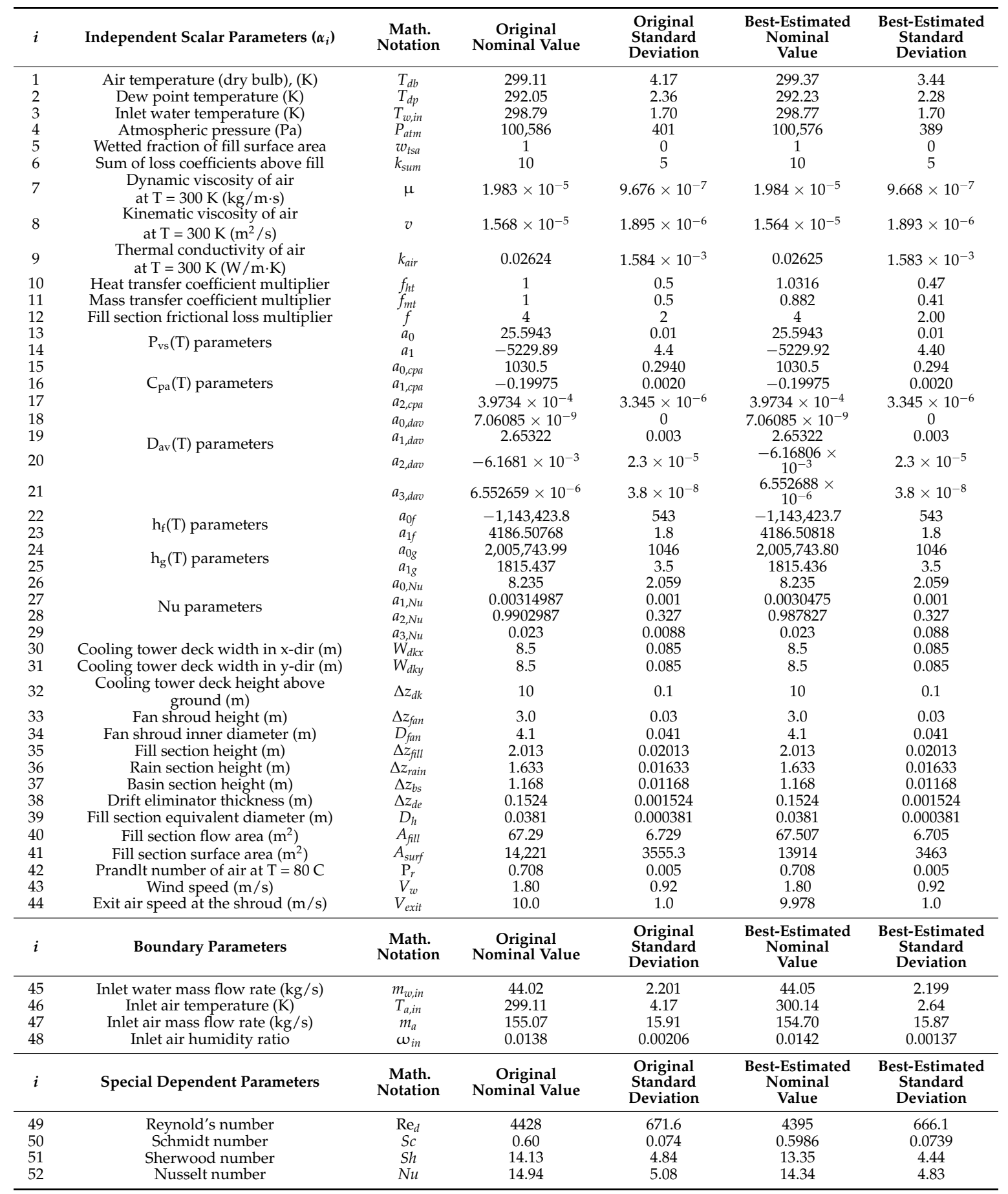


2.2.2. Predicted Best-Estimated Response Values with Reduced Predicted Standard Deviations

Using the a priori matrices given in Equations (20)-(23) together with the sensitivities presented in Tables 1-4 in Equation (18) yields the following predicted response covariance matrix, $\mathbf{C}_{r r}^{\text {pred }}$ :

$$
\mathbf{C}_{r r}^{p r e d} \equiv \operatorname{Cov}\left(\left[T_{a}^{(1)}\right]^{b e},\left[T_{w}^{(50)}\right]^{b e},\left[R H^{(1)}\right]^{b e}\right)=\left(\begin{array}{ccc}
6.71 & 2.73 & -22.80 \\
2.73 & 2.37 & -1.79 \\
-22.80 & -1.79 & 145.19
\end{array}\right) .
$$

The best-estimate response-parameter correlation matrix, $\mathbf{C}_{\alpha r}^{\text {pred }}$, is obtained using Equation (19) together with the a priori matrices given in Equations (20)-(23) and the sensitivities presented in Tables 1-4. The non-zero elements with the largest magnitudes are as follows:

$$
\begin{array}{lrl}
\text { rel.cor. }\left(R_{1}, \alpha_{4}\right)=-0.278 ; & \text { rel.cor. }\left(R_{1}, \alpha_{41}\right)=-0.070 ; & \text { rel.cor. }\left(R_{1}, \alpha_{49}\right)=-0.039 ; \\
\text { rel.cor. }\left(R_{2}, \alpha_{4}\right)=-0.108 ; & \text { rel.cor. }\left(R_{2}, \alpha_{41}\right)=-0.019 ; \\
r \text { rel.cor. }\left(R_{3}, \alpha_{4}\right)=0.232 ; & \text { rel.cor. }\left(R_{3}, \alpha_{41}\right)=0.127 ; \quad \text { rel.cor. }\left(R_{3}, \alpha_{49}\right)=0.072 .
\end{array}
$$

The notation used in Equation (25) is as follows: $R_{1} \equiv T_{a}^{(1)}, R_{2} \equiv T_{w}^{(50)}, R_{3} \equiv R H^{(1)}, \alpha_{4} \equiv P_{a t m}$, $\alpha_{41} \equiv A_{\text {surf }}$ and $\alpha_{49} \equiv \operatorname{Re}_{d}$.

The best-estimate nominal values of the (model responses) outlet air temperature, $T_{a}^{(1)}$; outlet water temperature $T_{w}^{(50)}$; and outlet air relative humidity, $R H^{(1)}$, have been computed using Equation (17) together with the a priori matrices given in Equations (20)-(23) and the sensitivities presented in Tables 1-4. The resulting best-estimate predicted nominal values are summarized in Table 6. To facilitate comparison, the corresponding measured and computed nominal values are also presented in this table. Note that there are no direct measurements for the outlet water flow rate, $m_{w}^{(50)}$. For this response, therefore, the predicted best-estimate nominal value has been obtained by a forward re-computation using the best-estimate nominal parameter values listed in Table 5, while the predicted best estimate standard deviation for this response has been obtained by using "best-estimate" values in Equation (15), i.e.:

$$
\left[\mathbf{C}_{r r}^{c o m p}\right]^{b e}=\left[\mathbf{S}_{r \alpha}\right]^{b e}\left[\mathbf{C}_{\alpha \alpha}\right]^{b e}\left[\mathbf{S}_{r \alpha}^{+}\right]^{b e} \text {. }
$$

Table 6. Computed, measured, and optimal best-estimate nominal values and standard deviations for the outlet air temperature, outlet water temperature, outlet air relative humidity, and outlet water flow rate responses.

\begin{tabular}{ccccc}
\hline Nominal Values and Standard Deviations & $T_{a}^{(1)}(\mathbf{K})$ & $T_{w}^{(50)}(\mathbf{K})$ & $R H^{(\mathbf{1})}(\%)$ & $m_{w}^{(50)}(\mathbf{k g} / \mathbf{s})$ \\
\hline Measured & & & & \\
\hline Nominal value & 298.34 & 295.68 & 81.98 & - \\
Standard deviation & \pm 3.36 & \pm 1.59 & \pm 15.89 & - \\
\hline Computed & & & & \\
\hline Nominal value & 297.46 & 294.58 & 86.12 & 43.60 \\
Standard deviation & \pm 3.30 & \pm 2.78 & \pm 14.90 & \pm 2.21 \\
\hline Best-estimate & & & & \\
\hline Nominal value & & & \\
Standard deviation & 298.45 & 295.67 & 82.12 & 43.67 \\
\hline & \pm 2.59 & \pm 1.54 & \pm 12.05 & \pm 2.20 \\
\hline
\end{tabular}

The results presented in Table 6 indicate that the predicted standard deviations are smaller than either the computed or the experimentally measured ones. This is indeed the consequence of using the PM_CMPS methodology in conjunction with consistent (as opposed to discrepant) computational and experimental information. Often, however, the information is inconsistent, usually due to the presence of unrecognized errors. Solutions for addressing such situations have been proposed in [10]. 
It is also important to note that the PM_CMPS methodology has improved (i.e., reduced, albeit not by a significant amount) the predicted standard deviation for the outlet water flow rate response, for which no measurements were available. This improvement stems from the global characteristics of the PM_CMPS methodology, which combines all of the available simultaneously on phase-space, as opposed to combining it sequentially, as is the case with the current state-of-the-art data assimilation procedures [11,12].

\section{Discussion}

In the present work, the adjoint sensitivity model of the counter-flow cooling tower derived in the accompanying PART I [1] was used to obtain the expressions and relative numerical rankings of the sensitivities, to all model parameters, of the following responses (quantities of interest): (i) the outlet air temperature; (ii) the outlet water temperature; (iii) the outlet water mass flow rate; and (iv) the air outlet relative humidity. These sensitivities were subsequently used within the "predictive modeling for coupled multi-physics systems" (PM_CMPS) methodology [2] to obtain explicit formulas for the predicted optimal nominal values for the model responses and parameters, along with reduced predicted standard deviations for the predicted model parameters and responses. These explicit formulas embody the assimilation of experimental data and the "calibration" of the model's parameters.

The results presented in this work indicate that the predicted standard deviations are smaller than either the computed or the experimentally measured ones. It is also important to note that the PM_CMPS methodology has improved (i.e., reduced, albeit not by a significant amount) the predicted standard deviation for the outlet water flow rate response, for which no measurements were available. This improvement stems from the global characteristics of the PM_CMPS methodology, which combines all of the available information simultaneously in phase-space, as opposed to combining it sequentially, as is the case with the current state-of-the-art data assimilation procedures [11,12]. This is indeed the consequence of using the PM_CMPS methodology in conjunction with consistent (as opposed to discrepant) computational and experimental information. Often, however, the information is inconsistent, usually due to the presence of unrecognized errors. Solutions for addressing such situations have been proposed in [10].

The adjoint sensitivity analysis methodology used in PART I [1] for computing exactly and efficiently the $1^{\text {st }}$-order response sensitivities to model parameters has been recently extended to computing efficiently and exactly the 2nd-order response sensitivities to parameters for linear [13] and nonlinear [14] large-scale systems. As has been shown in [15-18], the 2nd-order response sensitivities have the following major impacts on the computed moments of the response distribution: (a) they cause the "expected value of the response" to differ from the "computed nominal value of the response"; and (b) they contribute decisively to causing asymmetries in the response distribution. Indeed, neglecting the second-order sensitivities would nullify the third-order response correlations, and hence would nullify the skewness of the response. Consequently, non-Gaussian features (i.e., asymmetries, long-tails) any events occurring in a response's long and/or short tails, which are characteristic of rare but decisive events (e.g., major accidents, catastrophes), would likely be missed. Ongoing work aims at further applications and generalization of the adjoint sensitivity analysis and the PM_CMPS methodologies, to enable the computation of 3rd- and higher-order sensitivities and response distributions. The exact and efficient computation of high-order response sensitivities for large-scale systems is expected to advance significantly the areas of uncertainty quantification, model validation, reduced-order modeling, and predictive modeling/data assimilation.

Acknowledgments: This work has been partially sponsored by the US Department of Energy (James J. Peltz, Program manager) with the University of South Carolina.

Author Contributions: Ruixian Fang performed all of the numerical calculations in this paper. Dan Cacuci conceived and directed the research reported herein, and wrote the paper. Madalina Badea contributed the programming of the equations underlying the "predictive modeling" formalism.

Conflicts of Interest: The authors declare no conflict of interest. 


\section{Appendix A. Derivatives of Cooling Tower Model Equations with Respect To Model Parameters}

For convenience, the model parameters are reproduced in Table A1 below from Appendix B of PART I [1]. The independent model parameters are used for computing various dependent model parameters and thermal material properties, as shown in Tables A2 and A3, below.

Table A1. Parameters for SRNL f-area cooling towers.

\begin{tabular}{|c|c|c|c|c|c|c|}
\hline $\begin{array}{l}\operatorname{Index} i \\
\text { of } \alpha_{i}\end{array}$ & Independent Scalar Parameters & $\mathrm{C}++$ String & $\begin{array}{l}\text { Math. } \\
\text { Notation }\end{array}$ & $\begin{array}{l}\text { Nominal } \\
\text { Value(s) }\end{array}$ & $\begin{array}{l}\text { Absolute } \\
\text { Standard } \\
\text { Deviation }\end{array}$ & $\begin{array}{c}\text { Relative } \\
\text { Standard } \\
\text { Deviation (\%) }\end{array}$ \\
\hline 1 & Air temperature (dry bulb) (K) & $\mathrm{tdb}$ & $T_{d b}$ & 299.11 & 4.17 & 1.39 \\
\hline 2 & Dew point temperature $(\mathrm{K})$ & $\operatorname{tdp}$ & $T_{d p}$ & 292.05 & 2.36 & 0.81 \\
\hline 3 & Inlet water temperature (K) & twin & $T_{w, i n}$ & 298.79 & 1.70 & 0.57 \\
\hline 4 & Atmospheric pressure $(\mathrm{Pa})$ & patm & $P_{a t m}^{w, m}$ & 100586 & 401 & 0.40 \\
\hline 5 & Wetted fraction of fill surface area & wtsa & $w_{t s a}$ & 1 & 0 & 0 \\
\hline 6 & Sum of loss coefficients above fill & ksum & $k_{\text {sum }}$ & 10 & 5 & 50 \\
\hline 7 & $\begin{array}{l}\text { Dynamic viscosity of air } \\
\text { at } \mathrm{T}=300 \mathrm{~K}(\mathrm{~kg} / \mathrm{m} \cdot \mathrm{s})\end{array}$ & muair & $\mu$ & $1.983 \times 10^{-5}$ & $9.676 \times 10^{-7}$ & 4.88 \\
\hline 8 & $\begin{array}{l}\text { Kinematic viscosity of air } \\
\text { at } \mathrm{T}=300 \mathrm{~K}\left(\mathrm{~m}^{2} / \mathrm{s}\right)\end{array}$ & nuair & $v$ & $1.568 \times 10^{-5}$ & $1.895 \times 10^{-6}$ & 12.09 \\
\hline 9 & $\begin{array}{l}\text { Thermal conductivity of air } \\
\text { at } \mathrm{T}=300 \mathrm{~K}(\mathrm{~W} / \mathrm{m} \cdot \mathrm{K})\end{array}$ & tcair & $k_{\text {air }}$ & 0.02624 & $1.584 \times 10^{-3}$ & 6.04 \\
\hline 10 & Heat transfer coefficient multiplier & mlthtc & $f_{h t}$ & 1 & 0.5 & 50 \\
\hline 11 & Mass transfer coefficient multiplier & mltmtc & $f_{m t}$ & 1 & 0.5 & 50 \\
\hline 12 & Fill section frictional loss multiplier & mltfil & $f$ & 4 & 2 & 50 \\
\hline 13 & \multirow{3}{*}{$\mathrm{P}_{\mathrm{vs}}(\mathrm{T})$ parameters } & $\mathrm{a} 0$ & $a_{0}$ & 25.5943 & 0.01 & 0.04 \\
\hline 14 & & a1 & $a_{1}$ & -5229.89 & 4.4 & 0.08 \\
\hline 15 & & $\mathrm{~A}(1)$ & $a_{0, c p a}$ & 1030.5 & 0.2940 & 0.03 \\
\hline 16 & \multirow[t]{2}{*}{$\mathrm{C}_{\mathrm{pa}}(\mathrm{T})$ parameters } & $\mathrm{A}(2)$ & $a_{1, \text { cpa }}$ & -0.19975 & 0.0020 & 1.00 \\
\hline 17 & & $\mathrm{~A}(3)$ & $a_{2, c p a}$ & $3.9734 \times 10^{-4}$ & $3.345 \times 10^{-6}$ & 0.84 \\
\hline 18 & \multirow{4}{*}{$\mathrm{D}_{\mathrm{av}}(\mathrm{T})$ parameters } & $\mathrm{A}(1)$ & $a_{0, d a v}$ & $7.06085 \times 10^{-9}$ & 0 & 0 \\
\hline 19 & & $\mathrm{~A}(2)$ & $a_{1, \text { dav }}$ & 2.65322 & 0.003 & 0.11 \\
\hline 20 & & $\mathrm{~A}(3)$ & $a_{2, \text { dav }}$ & $-6.1681 \times 10^{-3}$ & $2.3 \times 10^{-5}$ & 0.37 \\
\hline 21 & & $\mathrm{~A}(4)$ & $a_{3, d a v}$ & $6.55266 \times 10^{-6}$ & $3.8 \times 10^{-8}$ & 0.58 \\
\hline 22 & \multirow{2}{*}{$h_{f}(T)$ parameters } & $\mathrm{aOf}$ & $a_{0 f}$ & $-1,143,423.78$ & 543. & 0.05 \\
\hline 23 & & a1f & $a_{1 f}$ & 4186.50768 & 1.8 & 0.04 \\
\hline 24 & & $\mathrm{a} 0 \mathrm{~g}$ & $a_{0 g}$ & $2,005,743.99$ & 1046 & 0.05 \\
\hline 25 & $h_{g}(1)$ parameters & a1g & $a_{1 g}$ & 1815.437 & 3.5 & 0.19 \\
\hline 26 & \multirow{4}{*}{ Nu parameters } & - & $a_{0, \mathrm{Nu}}$ & 8.235 & 2.059 & 25 \\
\hline 27 & & - & $a_{1, N u}$ & 0.00314987 & 0.001 & 31.75 \\
\hline 28 & & - & $a_{2, N u}$ & 0.9902987 & 0.327 & 33.02 \\
\hline 29 & & - & $a_{3, \mathrm{Nu}}^{2, v}$ & 0.023 & 0.0088 & 38.26 \\
\hline 30 & $\begin{array}{l}\text { Cooling tower deck } \\
\text { width in } x \text {-dir. }(\mathrm{m})\end{array}$ & $\mathrm{dkxw}$ & $W_{d k x}$ & 8.5 & 0.085 & 1 \\
\hline 31 & $\begin{array}{l}\text { Cooling tower deck } \\
\text { width in y-dir. }(\mathrm{m})\end{array}$ & dkyw & $W_{d k y}$ & 8.5 & 0.085 & 1 \\
\hline 32 & $\begin{array}{l}\text { Cooling tower deck } \\
\text { height above ground }(\mathrm{m})\end{array}$ & dkht & $\Delta z_{d k}$ & 10 & 0.1 & 1 \\
\hline 33 & Fan shroud height $(\mathrm{m})$ & fsht & $\Delta z_{f a n}$ & 3.0 & 0.03 & 1 \\
\hline 34 & Fan shroud inner diameter (m) & fsid & $D_{f a n}$ & 4.1 & 0.041 & 1 \\
\hline 35 & Fill section height $(\mathrm{m})$ & flht & $\Delta z_{\text {fill }}$ & 2.013 & 0.02013 & 1 \\
\hline 36 & Rain section height (m) & rsht & $\Delta z_{\text {rain }}$ & 1.633 & 0.01633 & 1 \\
\hline 37 & Basin section height (m) & bsht & $\Delta z_{b s}$ & 1.168 & 0.01168 & 1 \\
\hline 38 & \multirow{2}{*}{$\begin{array}{c}\text { Drift eliminator thickness }(\mathrm{m}) \\
\text { Fill section equivalent } \\
\text { diameter }(\mathrm{m})\end{array}$} & $\operatorname{detk}$ & $\Delta z_{d e}$ & 0.1524 & 0.001524 & 1 \\
\hline 39 & & deqv & $D_{h}$ & 0.0381 & 0.000381 & 1 \\
\hline 40 & Fill section flow area $\left(\mathrm{m}^{2}\right)$ & flfa & $A_{\text {fill }}$ & 67.29 & 6.729 & 10 \\
\hline 41 & Fill section surface area $\left(\mathrm{m}^{2}\right)$ & flsa & $A_{\text {surf }}$ & 14221 & 3555.3 & 25 \\
\hline 42 & Prandlt number of air at $\mathrm{T}=80 \mathrm{C}$ & $\operatorname{Pr}$ & $\mathrm{P}_{r}$ & 0.708 & 0.005 & 0.71 \\
\hline 43 & Wind speed $(\mathrm{m} / \mathrm{s})$ & wspd & $V_{w}$ & 1.80 & 0.92 & 51.1 \\
\hline 44 & Exit air speed at the shroud $(\mathrm{m} / \mathrm{s})$ & vexit & $V_{\text {exit }}$ & 10.0 & 1.0 & 10.0 \\
\hline $\begin{array}{l}\text { Index } i \\
\text { of } \alpha_{i}\end{array}$ & Boundary Parameters & $\mathrm{C}++$ String & $\begin{array}{l}\text { Math. } \\
\text { Notation }\end{array}$ & Nominal Value & $\begin{array}{l}\text { Absolute } \\
\text { Standard } \\
\text { Deviation }\end{array}$ & $\begin{array}{c}\text { Relative } \\
\text { Standard } \\
\text { Deviation (\%) }\end{array}$ \\
\hline 45 & Inlet water mass flow rate $(\mathrm{kg} / \mathrm{s})$ & mfwin & $m_{w, i n}$ & 44.02 & 2.201 & 5 \\
\hline 46 & Inlet air temperature $(\mathrm{K})$ & tain & $T_{a, i n}^{\infty, m}$ & set to $T_{d b}$ & 4.17 & 1.39 \\
\hline 47 & Inlet air mass flow rate $(\mathrm{kg} / \mathrm{s})$ & main & $m_{a}$ & 155.07 & 15.91 & 10.26 \\
\hline 48 & $\begin{array}{c}\text { Inlet air humidity ratio } \\
\text { (Dependent Scalar Parameter) }\end{array}$ & hrin & $\begin{array}{c}\omega_{\text {in }} \\
\omega_{\text {rain }}\end{array}$ & 0.0138 & 0.00206 & 14.93 \\
\hline 49 & $\begin{array}{l}\text { Reynold's number } \\
\text { Reymer) }\end{array}$ & $\operatorname{Re}$; Reh & $\begin{array}{l}\omega_{\text {rain }} \\
\operatorname{Re}_{d}\end{array}$ & 4428 & 671.6 & 15.17 \\
\hline 50 & Schmidt number & $\mathrm{Sc}$ & $S c$ & 0.60 & 0.074 & 12.41 \\
\hline 51 & Sherwood number & Sh & Sh & 14.13 & 4.84 & 34.25 \\
\hline 52 & Nusselt number & $\mathrm{Nu}$ & $\mathrm{Nu}$ & 14.94 & 5.08 & 34.00 \\
\hline
\end{tabular}


Table A2. Dependent scalar model parameters.

\begin{tabular}{|c|c|c|}
\hline Dependent Scalar Parameters & Math. Notation & Defining Equation or Correlation \\
\hline Mass diffusivity of water vapor in air $\left(\mathrm{m}^{2} / \mathrm{s}\right)$ & $D_{a v}\left(T_{a}, \boldsymbol{\alpha}\right)$ & $\frac{a_{0, d a v} T^{1.5}}{\left.a_{1}+a_{0}+a_{2} T\right) T}$ \\
\hline Heat transfer coefficient $\left(\mathrm{W} / \mathrm{m}^{2} \cdot \mathrm{K}\right)$ & $h(\boldsymbol{\alpha})$ & $\begin{array}{c}a_{1, d a v}+\left(a_{2, d a v}+a_{3, d a v} I\right) I \\
\frac{f_{h t} N_{n} k_{a i r}}{D_{h}}\end{array}$ \\
\hline Mass transfer coefficient $(\mathrm{m} / \mathrm{s})$ & $k_{m}(\boldsymbol{\alpha})$ & $\frac{f_{m t} S_{h} D_{h}}{D_{h}\left(T_{d b}, \alpha\right)}$ \\
\hline Heat transfer term $(\mathrm{W} / \mathrm{K})$ & $H\left(m_{a}, \alpha\right)$ & $h(\alpha) w_{t s a} A_{f f}$ \\
\hline Mass transfer term $\left(\mathrm{m}^{3} / \mathrm{s}\right)$ & $M\left(m_{a}, \alpha\right)$ & $M_{\mathrm{H}_{2} \mathrm{O}} k_{m}(\alpha) w_{t s a} A_{f f}$ \\
\hline Density of dry air $\left(\mathrm{kg} / \mathrm{m}^{3}\right)$ & $\rho(\alpha)$ & $\frac{P_{a t m}}{R_{a i r} T_{d h}}$ \\
\hline Air velocity in the fill section $(\mathrm{m} / \mathrm{s})$ & $v_{a}(m a, \alpha)$ & $\frac{\left|m_{a}\right|}{\rho(\alpha) A_{\text {fill }}}$ \\
\hline Fill falling-film surface area per vertical section $\left(\mathrm{m}^{2}\right)$ & $A_{f f}$ & $\frac{A_{\text {surf }}}{I}$ \\
\hline Rain section inlet flow area $\left(\mathrm{m}^{2}\right)$ & $A_{\text {in }}$ & $W_{d k x} W_{d k y}$ \\
\hline Height for natural convection (m) & Z & $z_{d k}+z_{f a n}-z_{b s}$ \\
\hline Height above fill section (m) & $\Delta z_{4-2}$ & $Z-z_{\text {fill }}-z_{\text {rain }}$ \\
\hline Fill section control volume height (m) & $\Delta z$ & $\frac{z_{\text {fill }}}{I}$ \\
\hline Fill section length, including drift eliminator (m) & $L_{\text {fill }}$ & $z_{\text {fill }}+z_{d e}$ \\
\hline Fan shroud inner radius $(\mathrm{m})$ & $r_{f a n}$ & $0.5 D_{f a n}$ \\
\hline Fan shroud flow area $\left(\mathrm{m}^{2}\right)$ & $A_{\text {out }}$ & $\pi r_{f a n}{ }^{2}$ \\
\hline
\end{tabular}

Table A3. Thermal properties (dependent scalar model parameters).

\begin{tabular}{|c|c|c|}
\hline Thermal Properties (Functions of State Variables) & Math. Notation & Defining Equation or Correlation \\
\hline $\mathrm{h}_{\mathrm{f}}\left(\mathrm{T}_{\mathrm{W}}\right)=$ saturated liquid enthalpy $(\mathrm{J} / \mathrm{kg})$ & $h_{f}\left(T_{w}, \boldsymbol{\alpha}\right)$ & $a_{0 f}+a_{1 f} T_{w}$ \\
\hline $\mathrm{H}_{\mathrm{g}}\left(\mathrm{T}_{\mathrm{w}}\right)=$ saturated vapor enthalpy $(\mathrm{J} / \mathrm{kg})$ & $h_{g, w}\left(T_{w}, \boldsymbol{\alpha}\right)$ & $a_{0 g}+a_{1 g} T_{w}$ \\
\hline $\mathrm{H}_{\mathrm{g}}\left(\mathrm{T}_{\mathrm{a}}\right)=$ saturated vapor enthalpy $(\mathrm{J} / \mathrm{kg})$ & $h_{g, a}\left(T_{a}, \alpha\right)$ & $a_{0 g}^{0}+a_{1 g} T_{a}$ \\
\hline $\mathrm{C}_{\mathrm{p}}(\mathrm{T})=$ specific heat of dry air $(\mathrm{J} / \mathrm{kg} \cdot \mathrm{K})$ & $C_{p}(T, \alpha)$ & $a_{0, c p a}+\left(a_{1, c p a}+a_{2, c p a} T\right) T$ \\
\hline $\mathrm{P}_{\mathrm{vs}}\left(\mathrm{T}_{\mathrm{W}}\right)=$ saturation pressure $(\mathrm{Pa})$ & $P_{v s}\left(T_{w}, \alpha\right)$ & $P_{c} \cdot e^{a_{0}+\frac{a_{1}}{T_{w}}}$, in which $P_{c}=1.0 \mathrm{~Pa}$ \\
\hline $\mathrm{P}_{\mathrm{vs}}\left(\mathrm{T}_{\mathrm{a}}\right)=$ saturation pressure $(\mathrm{Pa})$ & $P_{v s}\left(T_{a}, \alpha\right)$ & $P_{c} \cdot e^{a_{0}+\frac{a_{1}}{T_{a}}}$, in which $P_{c}=1.0 \mathrm{~Pa}$ \\
\hline
\end{tabular}

Note: The parameters $\alpha_{1}$ through $\alpha_{4}$ (i.e., the dry bulb air temperature, dew point temperature, inlet water temperature, and atmospheric pressure) were measured at the SRNL site at which the F-area cooling towers are located. Among the 8079 measured benchmark data sets [8], 7688 data sets are considered to represent "unsaturated conditions", which have been used to derive the statistical properties (means, variance and covariance, skewness and kurtosis) for these model parameters, as shown in Figures B1 through B4 and Tables B4 through B7 in Appendix B of PART I [1].

Recall that the cooling tower model comprises conservation balances representing mathematically the following physical phenomena: A. liquid continuity; B. liquid energy balance; C. water vapor continuity; D. air and water vapor energy balance. For easy reference, these conservation equations are reproduced below from Section 2 of PART I [1]:

A. Liquid continuity equations:

Control Volume $i=1$ :

$$
N_{1}^{(1)}\left(\mathbf{m}_{w}, \mathbf{T}_{w}, \mathbf{T}_{a}, \boldsymbol{\omega} ; \boldsymbol{\alpha}\right) \triangleq m_{w}^{(2)}-m_{w, i n}+\frac{M\left(m_{a}, \boldsymbol{\alpha}\right)}{\bar{R}}\left[\frac{P_{v s}^{(2)}\left(T_{w}^{(2)}, \boldsymbol{\alpha}\right)}{T_{w}^{(2)}}-\frac{\omega^{(1)} P_{a t m}}{T_{a}^{(1)}\left(0.622+\omega^{(1)}\right)}\right]=0
$$

(ii) Control Volumes $i=2, \ldots, I-1$ :

$$
N_{1}^{(i)}\left(\mathbf{m}_{w}, \mathbf{T}_{w}, \mathbf{T}_{a}, \boldsymbol{\omega} ; \boldsymbol{\alpha}\right) \triangleq m_{w}^{(i+1)}-m_{w}^{(i)}+\frac{M\left(m_{a}, \boldsymbol{\alpha}\right)}{\bar{R}}\left[\frac{P_{v s}^{(i+1)}\left(T_{w}^{(i+1)}, \boldsymbol{\alpha}\right)}{T_{w}^{(i+1)}}-\frac{\omega^{(i)} P_{a t m}}{T_{a}^{(i)}\left(0.622+\omega^{(i)}\right)}\right]=0 ;
$$

(iii) Control Volume $i=I$ :

$$
N_{1}^{(I)}\left(\mathbf{m}_{w}, \mathbf{T}_{w}, \mathbf{T}_{a}, \boldsymbol{\omega} ; \boldsymbol{\alpha}\right) \triangleq m_{w}^{(I+1)}-m_{w}^{(I)}+\frac{M\left(m_{a}, \boldsymbol{\alpha}\right)}{\bar{R}}\left[\frac{P_{v s}^{(I+1)}\left(T_{w}^{(I+1)}, \boldsymbol{\alpha}\right)}{T_{w}^{(I+1)}}-\frac{\omega^{(I)} P_{a t m}}{T_{a}^{(I)}\left(0.622+\omega^{(I)}\right)}\right]=0
$$

B. Liquid energy balance equations: 
Control Volume $i=1$ :

$$
\begin{aligned}
N_{2}^{(1)}\left(\mathbf{m}_{w}, \mathbf{T}_{w}, \mathbf{T}_{a}, \boldsymbol{\omega} ; \boldsymbol{\alpha}\right) \triangleq m_{w, i n} h_{f}\left(T_{w, i n}, \boldsymbol{\alpha}\right)-\left(T_{w}^{(2)}-T_{a}^{(1)}\right) H\left(m_{a}, \boldsymbol{\alpha}\right) \\
-m_{w}^{(2)} h_{f}^{(2)}\left(T_{w}^{(2)}, \boldsymbol{\alpha}\right)-\left(m_{w, i n}-m_{w}^{(2)}\right) h_{g, w}^{(2)}\left(T_{w}^{(2)}, \boldsymbol{\alpha}\right)=0
\end{aligned}
$$

(ii) Control Volumes $i=2, \ldots, I-1$ :

$$
\begin{aligned}
& N_{2}^{(i)}\left(\mathbf{m}_{w}, \mathbf{T}_{w}, \mathbf{T}_{a}, \omega ; \boldsymbol{\alpha}\right) \triangleq m_{w}^{(i)} h_{f}^{(i)}\left(T_{w}^{(i)}, \boldsymbol{\alpha}\right)-\left(T_{w}^{(i+1)}-T_{a}^{(i)}\right) H\left(m_{a}, \boldsymbol{\alpha}\right) \\
& \quad-m_{w}^{(i+1)} h_{f}^{(i+1)}\left(T_{w}^{(i+1)}, \boldsymbol{\alpha}\right)-\left(m_{w}^{(i)}-m_{w}^{(i+1)}\right) h_{g, w}^{(i+1)}\left(T_{w}^{(i+1)}, \boldsymbol{\alpha}\right)=0
\end{aligned}
$$

(iii) Control Volume $i=I$ :

$$
\begin{aligned}
& N_{2}^{(I)}\left(\mathbf{m}_{w}, \mathbf{T}_{w}, \mathbf{T}_{a}, \boldsymbol{\omega} ; \boldsymbol{\alpha}\right) \triangleq m_{w}^{(I)} h_{f}^{(I)}\left(T_{w}^{(I)}, \boldsymbol{\alpha}\right)-\left(T_{w}^{(I+1)}-T_{a}^{(I)}\right) H\left(m_{a}, \boldsymbol{\alpha}\right) \\
& \quad-m_{w}^{(I+1)} h_{f}^{(I+1)}\left(T_{w}^{(I+1)}, \boldsymbol{\alpha}\right)-\left(m_{w}^{(I)}-m_{w}^{(I+1)}\right) h_{g, w}^{(I+1)}\left(T_{w}^{(I+1)}, \boldsymbol{\alpha}\right)=0
\end{aligned}
$$

C. Water vapor continuity equations:

(i) Control Volume $i=1$ :

$$
N_{3}^{(1)}\left(\mathbf{m}_{w}, \mathbf{T}_{w}, \mathbf{T}_{a}, \boldsymbol{\omega} ; \boldsymbol{\alpha}\right) \triangleq \omega^{(2)}-\omega^{(1)}+\frac{m_{w . i n}-m_{w}^{(2)}}{\left|m_{a}\right|}=0 ;
$$

(ii) Control Volumes $i=2, \ldots, I-1$ :

$$
N_{3}^{(i)}\left(\mathbf{m}_{w}, \mathbf{T}_{w}, \mathbf{T}_{a}, \boldsymbol{\omega} ; \boldsymbol{\alpha}\right) \triangleq \omega^{(i+1)}-\omega^{(i)}+\frac{m_{w}^{(i)}-m_{w}^{(i+1)}}{\left|m_{a}\right|}=0
$$

(iii) Control Volume $i=I$ :

$$
N_{3}^{(I)}\left(\mathbf{m}_{w}, \mathbf{T}_{w}, \mathbf{T}_{a}, \boldsymbol{\omega} ; \boldsymbol{\alpha}\right) \triangleq \omega_{i n}-\omega^{(I)}+\frac{m_{w}^{(I)}-m_{w}^{(I+1)}}{\left|m_{a}\right|}=0 ;
$$

D. The air/water vapor energy balance equations:

(i) Control Volume $i=1$ :

$$
\begin{gathered}
N_{4}^{(1)}\left(\mathbf{m}_{w}, \mathbf{T}_{w}, \mathbf{T}_{a}, \boldsymbol{\omega} ; \boldsymbol{\alpha}\right) \triangleq\left(T_{a}^{(2)}-T_{a}^{(1)}\right) C_{p}^{(1)}\left(\frac{T_{a}^{(1)}+273.15}{2}, \boldsymbol{\alpha}\right)-\omega^{(1)} h_{g, a}^{(1)}\left(T_{a}^{(1)}, \boldsymbol{\alpha}\right) \\
+\frac{\left(T_{w}^{(2)}-T_{a}^{(1)}\right) H\left(m_{a}, \boldsymbol{\alpha}\right)}{\left|m_{a}\right|}+\frac{\left(m_{w, i n}-m_{w}^{(2)}\right) h_{g, w}^{(2)}\left(T_{w}^{(2)}, \boldsymbol{\alpha}\right)}{\left|m_{a}\right|}+\omega^{(2)} h_{g, a}^{(2)}\left(T_{a}^{(2)}, \boldsymbol{\alpha}\right)=0
\end{gathered}
$$

(ii) Control Volumes $i=2, \ldots, I-1$ :

$$
\begin{aligned}
& N_{4}^{(i)}\left(\mathbf{m}_{w}, \mathbf{T}_{w}, \mathbf{T}_{a}, \omega ; \boldsymbol{\alpha}\right) \triangleq\left(T_{a}^{(i+1)}-T_{a}^{(i)}\right) C_{p}^{(i)}\left(\frac{T_{a}^{(i)}+273.15}{2}, \boldsymbol{\alpha}\right)-\omega^{(i)} h_{g, a}^{(i)}\left(T_{a}^{(i)}, \boldsymbol{\alpha}\right) \\
& +\frac{\left(T_{w}^{(i+1)}-T_{a}^{(i)}\right) H\left(m_{a}, \boldsymbol{\alpha}\right)}{\left|m_{a}\right|}+\frac{\left(m_{w}^{(i)}-m_{w}^{(i+1)}\right) h_{g, w}^{(i+1)}\left(T_{w}^{(i+1)}, \boldsymbol{\alpha}\right)}{\left|m_{a}\right|}+\omega^{(i+1)} h_{g, a}^{(i+1)}\left(T_{a}^{(i+1)}, \boldsymbol{\alpha}\right)=0
\end{aligned}
$$

(iii) Control Volume $i=I$ :

$$
\begin{gathered}
N_{4}^{(I)}\left(\mathbf{m}_{w}, \mathbf{T}_{w}, \mathbf{T}_{a}, \boldsymbol{\omega} ; \boldsymbol{\alpha}\right) \triangleq\left(T_{a, i n}-T_{a}^{(I)}\right) C_{p}{ }^{(I)}\left(\frac{T_{a}^{(I)}+273.15}{2}, \boldsymbol{\alpha}\right)-\omega^{(I)} h_{g, a}^{(I)}\left(T_{a}^{(I)}, \boldsymbol{\alpha}\right) \\
+\frac{\left(T_{w}^{(I+1)}-T_{a}^{(I)}\right) H\left(m_{a}, \boldsymbol{\alpha}\right)}{\left|m_{a}\right|}+\frac{\left(m_{w}^{(I)}-m_{w}^{(I+1)}\right) h_{g, w}^{(I+1)}\left(T_{w}^{(I+1)}, \boldsymbol{\alpha}\right)}{\left|m_{a}\right|}+\omega_{i n} h_{g, a}\left(T_{a, i n}, \boldsymbol{\alpha}\right)=0 .
\end{gathered}
$$


The components of the vector $\boldsymbol{\alpha}$, which appears in Equations (A1)-(A12), comprise the model parameters, i.e.:

$$
\alpha \triangleq\left(\alpha_{1}, \ldots, \alpha_{N_{\alpha}}\right)
$$

where $N_{\alpha}$ denotes the total number of model parameters. These model parameters are described in Table A1.

The following notation will be used for the derivatives of the above equations with respect to the parameters:

$$
a_{\ell}^{i, j} \equiv \frac{\partial N_{\ell}^{(i)}}{\partial \alpha(j)} ; \ell=1,2,3,4 ; i=1, \ldots, I ; j=1, \ldots, N_{\alpha} .
$$

\section{A1. Derivatives of the Liquid Continuity Equations with Respect to the Parameters}

The derivatives of the "liquid continuity equations" [cf. Equations (A1)-(A3)] with respect to the parameter $\alpha^{(1)} \equiv T_{d b}$ are as follows:

$$
\begin{gathered}
\frac{\partial N_{1}^{(i)}}{\partial \alpha^{(1)}}=\frac{\partial N_{1}^{(i)}}{\partial T_{d b}} \equiv a_{1}^{i, 1}=\frac{1}{\bar{R}}\left[\frac{P_{v s}^{(i+1)}\left(T_{w}^{(i+1)}, \boldsymbol{\alpha}\right)}{T_{w}^{(i+1)}}-\frac{\omega^{(i)} P_{a t m}}{\left(0.622+\omega^{(i)}\right) T_{a}^{(i)}}\right] \frac{\partial M\left(m_{a}, \boldsymbol{\alpha}\right)}{\partial D_{a v}\left(T_{d b}, \boldsymbol{\alpha}\right)} \cdot \frac{\partial D_{a v}\left(T_{d b}, \boldsymbol{\alpha}\right)}{\partial T_{d b}} \\
\ell=1 ; i=1, \ldots, I ; j=1
\end{gathered}
$$

where:

$$
\begin{gathered}
\frac{\partial M\left(m_{a}, \boldsymbol{\alpha}\right)}{\partial D_{a v}\left(T_{d b}, \boldsymbol{\alpha}\right)}=\frac{2}{3} \cdot \frac{M\left(m_{a}, \boldsymbol{\alpha}\right)}{D_{a v}\left(T_{d b}, \boldsymbol{\alpha}\right)} \\
\frac{\partial D_{a v}\left(T_{d b}, \boldsymbol{\alpha}\right)}{\partial T_{d b}}=\frac{1.5 \cdot a_{0 d a v} T_{d b} 0.5-D_{a v}\left(T_{d b}, \boldsymbol{\alpha}\right) \cdot\left(a_{2 d a v}+2 \cdot a_{3 d a v} T_{d b}\right)}{a_{1 d a v}+a_{2 d a v} T_{d b}+a_{3 d a v} T_{d b}{ }^{2}}
\end{gathered}
$$

The derivatives of the "liquid continuity equations" [cf. Equations (A1)-(A3)] with respect to the parameter $\alpha^{(2)} \equiv T_{d p}$ are as follows:

$$
\frac{\partial N_{1}^{(i)}}{\partial \alpha^{(2)}}=\frac{\partial N_{1}^{(i)}}{\partial T_{d p}} \equiv a_{1}^{i, 2}=0 ; \quad \ell=1 ; i=1, \ldots, I ; j=2 .
$$

The derivatives of the "liquid continuity equations" [cf. Equations (A1)-(A3)] with respect to the parameter $\alpha^{(3)} \equiv T_{w, \text { in }}$ are as follows:

$$
\frac{\partial N_{1}^{(1)}}{\partial \alpha^{(3)}}=\frac{\partial N_{1}^{(1)}}{\partial T_{w, i n}} \equiv a_{1}^{1,3}=-\frac{\partial m_{w, i n}}{\partial T_{w, i n}} ; \quad \ell=1 ; i=1 ; j=3,
$$

where:

$$
\begin{aligned}
& \frac{\partial m_{w, \text { in }}}{\partial T_{w, i n}}=\frac{\partial}{\partial T_{w, i n}}\left[\rho\left(T_{w, \text { in }}\right) \cdot \frac{700.0}{15850.32}\right] \\
& =\left[a_{2, \rho}+2 \cdot a_{3, \rho}\left(T_{w, \text { in }}-273.15\right)+3 \cdot a_{4, \rho}\left(T_{w, \text { in }}-273.15\right)^{2}\right] \cdot \frac{700.0}{15850.32} ;
\end{aligned}
$$

and where $a_{2, \rho}=-0.26847207 ; a_{3, \rho}=-1.8113691 \times 10^{-3} ; a_{4, \rho}=-1.7041217 \times 10^{-6}$.

$$
\frac{\partial N_{1}^{(i)}}{\partial \alpha^{(3)}}=\frac{\partial N_{1}^{(i)}}{\partial T_{w, i n}} \equiv a_{1}^{i, 3}=0 ; \quad \ell=1 ; i=2, \ldots, I ; j=3 .
$$

The derivatives of the "liquid continuity equations" [cf. Equations (A1)-(A3)] with respect to the parameter $\alpha^{(4)} \equiv P_{\text {atm }}$ are as follows:

$$
\begin{aligned}
\frac{\partial N_{1}^{(i)}}{\partial \alpha^{(4)}} & =\frac{\partial N_{1}^{(i)}}{\partial P_{a t m}} \equiv a_{1}^{i, 4}=-\frac{M\left(m_{a}, \alpha\right)}{\bar{R}} \frac{\omega^{(i)}}{T_{a}^{(i)}\left(0.622+\omega^{(i)}\right)} \\
& +\frac{1}{\bar{R}}\left[\frac{P_{v s}^{(i+1)}\left(T_{w}^{(i+1)}, \alpha\right)}{T_{w w}^{(i+1)}}-\frac{\omega^{(i)} P_{a t m}}{\left(0.622+\omega^{(i)}\right) T_{a}^{(i)}}\right] \frac{\partial M\left(m_{a}, \alpha\right)}{\partial N u(\operatorname{Re}, \alpha)} \frac{\partial N u(\operatorname{Re}, \alpha)}{\partial m_{a}} \frac{\partial m_{a}}{\partial P_{a t m}} ; \\
& \quad=1 ; i=1, \ldots, I ; j=4,
\end{aligned}
$$


where:

$$
\begin{gathered}
\frac{\partial M(\operatorname{Re}, \boldsymbol{\alpha})}{\partial N u(\operatorname{Re}, \boldsymbol{\alpha})}=\frac{M\left(m_{a}, \boldsymbol{\alpha}\right)}{N u(\operatorname{Re}, \boldsymbol{\alpha})}, \\
\frac{\partial N u(\operatorname{Re}, \boldsymbol{\alpha})}{\partial m_{a}}=\left\{\begin{array}{cc}
0 & \operatorname{Re}_{d}<2300 \\
a_{1, N u} \operatorname{Re}\left(m_{a}, \boldsymbol{\alpha}\right) / m_{a} & 2300 \leq \operatorname{Re}_{d} \leq 10000 \\
0.8 N u(\operatorname{Re}, \boldsymbol{\alpha}) / m_{a} & \operatorname{Re}_{d}>10000
\end{array}\right. \\
\frac{\partial m_{a}}{\partial P_{a t m}}=\frac{1}{R_{a i r} T_{a, i n}} \cdot V_{\text {exit }} \cdot \frac{\pi D_{\text {fan }}{ }^{2}}{4} ;
\end{gathered}
$$

Note: The term on the right hand side of Equation (A25) stems from the following relation:

$$
m_{a}=\rho\left(T_{a}\right) \cdot V_{\text {exit }} \cdot \frac{\pi D_{\text {fan }}^{2}}{4}=\frac{P_{a t m}}{R_{a i r} T_{a}} \cdot V_{\text {exit }} \cdot \frac{\pi D_{\text {fan }}{ }^{2}}{4} .
$$

The derivatives of the "liquid continuity equations" [cf. Equations (A1)-(A3)] with respect to the parameter $\alpha^{(5)} \equiv w_{t s a}$ are as follows:

$$
\begin{gathered}
\frac{\partial N_{1}^{(i)}}{\partial \alpha^{(5)}}=\frac{\partial N_{1}^{(i)}}{\partial w_{t s a}} \equiv a_{1}^{i, 5}=\frac{1}{R}\left[\frac{P_{v s}^{(i+1)}\left(T_{w}^{(i+1)}, \alpha\right)}{T_{w}^{(i+1)}}-\frac{\omega^{(i)} P_{a t m}}{\left(0.622+\omega^{(i)}\right) T_{a}^{(i)}}\right] \frac{\partial M\left(m_{a}, \alpha\right)}{\partial w_{t s a}} ; \\
\ell=1 ; i=1, \ldots, I ; j=5,
\end{gathered}
$$

where:

$$
\frac{\partial M\left(m_{a}, \boldsymbol{\alpha}\right)}{\partial w_{t s a}}=\frac{M_{H 2 O} f_{m t} N u(\operatorname{Re}, \boldsymbol{\alpha})\left(\frac{v}{\operatorname{Pr}}\right)^{\frac{1}{3}}\left[D_{a v}\left(T_{d b}, \boldsymbol{\alpha}\right)\right]^{\frac{2}{3}} A_{\text {surf }}}{\mathrm{D}_{h} I}
$$

The derivatives of the "liquid continuity equations" [cf. Equations (A1)-(A3)] with respect to the parameter $\alpha^{(6)} \equiv k_{\text {sum }}$ are as follows:

$$
\frac{\partial N_{1}^{(i)}}{\partial \alpha^{(6)}}=\frac{\partial N_{1}^{(i)}}{\partial k_{\text {sum }}} \equiv a_{1}^{i, 6}=0 ; \quad \ell=1 ; i=1, \ldots, I ; j=6 .
$$

The derivatives of the "liquid continuity equations" [cf. Equations (A1)-(A3)] with respect to the parameter $\alpha^{(7)} \equiv \mu$ are as follows:

$$
\begin{gathered}
\frac{\partial N_{1}^{(i)}}{\partial \alpha^{(7)}}=\frac{\partial N_{1}^{(i)}}{\partial \mu} \equiv a_{1}^{i, 7}=\frac{1}{R}\left[\frac{P_{v s}^{(i+1)}\left(T_{w}^{(i+1)}, \alpha\right)}{T_{w}^{(i+1)}}-\frac{\omega^{(i)} P_{a t m}}{\left(0.622+\omega^{(i)}\right) T_{a}^{(i)}}\right] \frac{\partial M\left(m_{a}, \alpha\right)}{\partial \mu} \\
\ell=1 ; i=1, \ldots, I ; j=7,
\end{gathered}
$$

where:

$$
\frac{\partial M\left(m_{a}, \boldsymbol{\alpha}\right)}{\partial \mu}= \begin{cases}0 & \operatorname{Re}_{d}<2300 \\ -\frac{a_{1, N u} \cdot M\left(m_{a}, \alpha\right) \cdot \operatorname{Re}\left(m_{a}, \alpha\right)}{N u(\operatorname{Re}, \boldsymbol{\alpha}) \cdot \mu} & 2300 \leq \operatorname{Re}_{d} \leq 10000 \\ -0.8 \cdot \frac{M\left(m_{a}, \boldsymbol{\alpha}\right)}{\mu} & \operatorname{Re}_{d}>10000\end{cases}
$$

The derivatives of the "liquid continuity equations" [cf. Equations (A1)-(A3)] with respect to the parameter $\alpha^{(8)} \equiv v$ are as follows:

$$
\begin{gathered}
\frac{\partial N_{1}^{(i)}}{\partial \alpha^{(8)}}=\frac{\partial N_{1}^{(i)}}{\partial v} \equiv a_{1}^{i, 8}=\frac{1}{R}\left[\frac{P_{v s}^{(i+1)}\left(T_{w}^{(i+1)}, \alpha\right)}{T_{w}^{(i+1)}}-\frac{\omega^{(i)} P_{a t m}}{\left(0.622+\omega^{(i)}\right) T_{a}^{(i)}}\right] \frac{\partial M\left(m_{a}, \alpha\right)}{\partial v} ; \\
\ell=1 ; i=1, \ldots, I ; j=8,
\end{gathered}
$$

where:

$$
\frac{\partial M\left(m_{a}, \boldsymbol{\alpha}\right)}{\partial v}=\frac{1}{3} \frac{M\left(m_{a}, \boldsymbol{\alpha}\right)}{v}
$$


The derivatives of the "liquid continuity equations" [cf. Equations (A1)-(A3)] with respect to the parameter $\alpha^{(9)} \equiv k_{\text {air }}$ are as follows:

$$
\frac{\partial N_{1}^{(i)}}{\partial \alpha^{(9)}}=\frac{\partial N_{1}^{(i)}}{\partial k_{\text {air }}} \equiv a_{1}^{i, 9}=0 ; \quad \ell=1 ; i=1, \ldots, I ; j=9 .
$$

The derivatives of the "liquid continuity equations" [cf. Equations (A1)-(A3)] with respect to the parameter $\alpha^{(10)} \equiv f_{h t}$ are as follows:

$$
\frac{\partial N_{1}^{(i)}}{\partial \alpha^{(10)}}=\frac{\partial N_{1}^{(i)}}{\partial f_{h t}} \equiv a_{1}^{i, 10}=0 ; \quad \ell=1 ; i=1, \ldots, I ; j=10 .
$$

The derivatives of the "liquid continuity equations" [cf. Equations (A1)-(A3)] with respect to the parameter $\alpha^{(11)} \equiv f_{m t}$ are as follows:

$$
\begin{gathered}
\frac{\partial N_{1}^{(i)}}{\partial \alpha^{(11)}}=\frac{\partial N_{1}^{(i)}}{\partial f_{m t}} \equiv a_{1}^{i, 11}=\frac{1}{\bar{R}}\left[\frac{P_{v s}^{(i+1)}\left(T_{w}^{(i+1)}, \alpha\right)}{T_{w}^{(i+1)}}-\frac{\omega^{(i)} P_{a t m}}{\left(0.622+\omega^{(i)}\right) T_{a}^{(i)}}\right] \frac{\partial M\left(m_{a}, \alpha\right)}{\partial f_{m t}} ; \\
\ell=1 ; i=1, \ldots, I ; j=11
\end{gathered}
$$

where:

$$
\frac{\partial M\left(m_{a}, \boldsymbol{\alpha}\right)}{\partial f_{m t}}=\frac{M_{H 2 O} N u(\operatorname{Re}, \boldsymbol{\alpha})\left(\frac{v}{\operatorname{Pr}}\right)^{\frac{1}{3}}\left[D_{a v}\left(T_{d b}, \boldsymbol{\alpha}\right)\right]^{\frac{2}{3}} w_{t s a} A_{\text {surf }}}{\mathrm{D}_{h} I} .
$$

The derivatives of the "liquid continuity equations" [cf. Equations (A1)-(A3)] with respect to the parameter $\alpha^{(12)} \equiv f$ are as follows:

$$
\frac{\partial N_{1}^{(i)}}{\partial \alpha^{(12)}}=\frac{\partial N_{1}^{(i)}}{\partial f} \equiv a_{1}^{i, 12}=0 ; \quad \ell=1 ; i=1, \ldots, I ; j=12 .
$$

The derivatives of the "liquid continuity equations" [cf. Equations (A1)-(A3)] with respect to the parameter $\alpha^{(13)} \equiv a_{0}$ are as follows:

$$
\frac{\partial N_{1}^{(i)}}{\partial \alpha^{(13)}}=\frac{\partial N_{1}^{(i)}}{\partial a_{0}} \equiv a_{1}^{i, 13}=\frac{M\left(m_{a}, \alpha\right)}{\bar{R}} \frac{1}{T_{w}^{(i+1)}} \frac{\partial P_{v s}^{(i+1)}\left(T_{w}^{(i+1)}, \alpha\right)}{\partial a_{0}} ; \quad \ell=1 ; i=1, \ldots, I ; j=13,
$$

where:

$$
\frac{\partial P_{v s}^{(i+1)}\left(T_{w}^{(i+1)}, \boldsymbol{\alpha}\right)}{\partial a_{0}}=P_{v s}^{(i+1)}\left(T_{w}^{(i+1)}, \boldsymbol{\alpha}\right) .
$$

The derivatives of the "liquid continuity equations" [cf. Equations (A1)-(A3)] with respect to the parameter $\alpha^{(14)} \equiv a_{1}$ are as follows:

$$
\begin{aligned}
\frac{\partial N_{1}^{(i)}}{\partial \alpha^{(14)}}=\frac{\partial N_{1}^{(i)}}{\partial a_{1}} & \equiv a_{1}^{i, 14}=\frac{M\left(m_{a}, \alpha\right)}{\bar{R}} \frac{1}{T_{w}^{(i+1)}} \frac{\partial P_{v s}^{(i+1)}\left(T_{w o}^{(i+1)}, \alpha\right)}{\partial a_{1}} \\
\ell & =1 ; i=1, \ldots, I ; j=14
\end{aligned}
$$

where:

$$
\frac{\partial P_{v s}^{(i+1)}\left(T_{w}^{(i+1)}, \alpha\right)}{\partial a_{1}}=\frac{P_{v s}^{(i+1)}\left(T_{w}^{(i+1)}, \alpha\right)}{T_{w}^{(i+1)}} .
$$

The derivatives of the "liquid continuity equations" [cf. Equations (A1)-(A3)] with respect to the parameter $\alpha^{(15)} \equiv a_{0, \text { cpa }}$ are as follows:

$$
\frac{\partial N_{1}^{(i)}}{\partial \alpha^{(15)}}=\frac{\partial N_{1}^{(i)}}{\partial a_{0, c p a}} \equiv a_{1}^{i, 15}=0 ; \quad \ell=1 ; i=1, \ldots, I ; j=15 .
$$


The derivatives of the "liquid continuity equations" [cf. Equations (A1)-(A3)] with respect to the parameter $\alpha^{(16)} \equiv a_{1, \text { cpa }}$ are as follows:

$$
\frac{\partial N_{1}^{(i)}}{\partial \alpha^{(16)}}=\frac{\partial N_{1}^{(i)}}{\partial a_{1, c p a}} \equiv a_{1}^{i, 16}=0 ; \quad \ell=1 ; i=1, \ldots, I ; j=16 .
$$

The derivatives of the "liquid continuity equations" [cf. Equations (A1)-(A3)] with respect to the parameter $\alpha^{(17)} \equiv a_{2, \text { cpa }}$ are as follows:

$$
\frac{\partial N_{1}^{(i)}}{\partial \alpha^{(17)}}=\frac{\partial N_{1}^{(i)}}{\partial a_{2, c p a}} \equiv a_{1}^{i, 17}=0 ; \quad \ell=1 ; i=1, \ldots, I ; j=17 .
$$

The derivatives of the "liquid continuity equations" [cf. Equations (A1)-(A3)] with respect to the parameter $\alpha^{(18)} \equiv a_{0, d a v}$ are as follows:

$$
\begin{gathered}
\frac{\partial N_{1}^{(i)}}{\partial \alpha^{(18)}}=\frac{\partial N_{1}^{(i)}}{\partial a_{0, d a v}} \equiv a_{1}^{i, 18}=\frac{1}{\bar{R}}\left[\frac{P_{v s}^{(i+1)}\left(T_{w}^{(i+1)}, \boldsymbol{\alpha}\right)}{T_{w}^{(i+1)}}-\frac{\omega^{(i)} P_{a t m}}{\left(0.622+\omega^{(i)}\right) T_{a}^{(i)}}\right] \frac{\partial M\left(m_{a}, \boldsymbol{\alpha}\right)}{\partial D_{a v}\left(T_{d b}, \boldsymbol{\alpha}\right)} \cdot \frac{\partial D_{a v}\left(T_{d b}, \boldsymbol{\alpha}\right)}{\partial a_{0, d a v}} \\
\ell=1 ; i=1, \ldots, I ; j=18
\end{gathered}
$$

where $\frac{\partial M\left(m_{a}, \alpha\right)}{\partial D_{a v}\left(T_{d b}, \alpha\right)}$ was defined previously in Equation (A16), and:

$$
\frac{\partial D_{a v}\left(T_{d b}, \boldsymbol{\alpha}\right)}{\partial a_{0, d a v}}=\frac{T_{d b}^{1.5}}{a_{1 d a v}+a_{2 d a v} T_{d b}+a_{3 d a v} T_{d b}^{2}} .
$$

The derivatives of the "liquid continuity equations" [cf. Equations (A1)-(A3)] with respect to the parameter $\alpha^{(19)} \equiv a_{1, \text { dav }}$ are as follows:

$$
\begin{gathered}
\frac{\partial N_{1}^{(i)}}{\partial \alpha^{(19)}}=\frac{\partial N_{1}^{(i)}}{\partial a_{1, d a v}} \equiv a_{1}^{i, 19}=\frac{1}{\bar{R}}\left[\frac{P_{v s}^{(i+1)}\left(T_{w}^{(i+1)}, \boldsymbol{\alpha}\right)}{T_{w}^{(i+1)}}-\frac{\omega^{(i)} P_{a t m}}{\left(0.622+\omega^{(i)}\right) T_{a}^{(i)}}\right] \frac{\partial M\left(m_{a}, \boldsymbol{\alpha}\right)}{\partial D_{a v}\left(T_{d b}, \boldsymbol{\alpha}\right)} \cdot \frac{\partial D_{a v}\left(T_{d b}, \boldsymbol{\alpha}\right)}{\partial a_{1, d a v}} \\
\ell=1 ; i=1, \ldots, I ; j=19
\end{gathered}
$$

where $\frac{\partial M\left(m_{a}, \alpha\right)}{\partial D_{a v}\left(T_{d b}, \alpha\right)}$ was defined previously in Equation (A16), and:

$$
\frac{\partial D_{a v}\left(T_{d b}, \boldsymbol{\alpha}\right)}{\partial a_{1, d a v}}=-\frac{a_{0 d a v} T_{d b}^{1.5}}{\left(a_{1 d a v}+a_{2 d a v} T_{d b}+a_{3 d a v} T_{d b}^{2}\right)^{2}} .
$$

The derivatives of the "liquid continuity equations" [cf. Equations (A1)-(A3)] with respect to the parameter $\alpha^{(20)} \equiv a_{2, \text { dav }}$ are as follows:

$$
\begin{gathered}
\frac{\partial N_{1}^{(i)}}{\partial \alpha^{(20)}}=\frac{\partial N_{1}^{(i)}}{\partial a_{2, d a v}} \equiv a_{1}^{i, 20}=\frac{1}{\bar{R}}\left[\frac{P_{v s}^{(i+1)}\left(T_{w}^{(i+1)}, \boldsymbol{\alpha}\right)}{T_{w w}^{(i+1)}}-\frac{\omega^{(i)} P_{a t m}}{\left(0.622+\omega^{(i)}\right) T_{a}^{(i)}}\right] \frac{\partial M\left(m_{a}, \boldsymbol{\alpha}\right)}{\partial D_{a v}\left(T_{d b}, \boldsymbol{\alpha}\right)} \cdot \frac{\partial D_{a v}\left(T_{d b}, \boldsymbol{\alpha}\right)}{\partial a_{2, d a v}} \\
\ell=1 ; i=1, \ldots, I ; j=20
\end{gathered}
$$

where $\frac{\partial M\left(m_{a}, \alpha\right)}{\partial D_{a v}\left(T_{d b}, \alpha\right)}$ was defined previously in Equation (A16), and

$$
\frac{\partial D_{a v}\left(T_{d b}, \boldsymbol{\alpha}\right)}{\partial a_{2, d a v}}=-\frac{a_{0 d a v} T_{d b}^{2.5}}{\left(a_{1 d a v}+a_{2 d a v} T_{d b}+a_{3 d a v} T_{d b}^{2}\right)^{2}} .
$$


The derivatives of the "liquid continuity equations" [cf. Equations (A1)-(A3)] with respect to the parameter $\alpha^{(21)} \equiv a_{3, \text { dav }}$ are as follows:

$$
\begin{gathered}
\frac{\partial N_{1}^{(i)}}{\partial \alpha^{(21)}}=\frac{\partial N_{1}^{(i)}}{\partial a_{3, d a v}} \equiv a_{1}^{i, 21}=\frac{1}{R}\left[\frac{P_{v s}^{(i+1)}\left(T_{w}^{(i+1)}, \boldsymbol{\alpha}\right)}{T_{w}^{(i+1)}}-\frac{\omega^{(i)} P_{a t m}}{\left(0.622+\omega^{(i)}\right) T_{a}^{(i)}}\right] \frac{\partial M\left(m_{a}, \boldsymbol{\alpha}\right)}{\partial D_{a v}\left(T_{d b}, \boldsymbol{\alpha}\right)} \cdot \frac{\partial D_{a v}\left(T_{d b}, \boldsymbol{\alpha}\right)}{\partial a_{3, d a v}} ; \\
\ell=1 ; i=1, \ldots, I ; j=21,
\end{gathered}
$$

where $\frac{\partial M\left(m_{a}, \alpha\right)}{\partial D_{a v}\left(T_{d b}, \alpha\right)}$ was defined previously in Equation (A16), and

$$
\frac{\partial D_{a v}\left(T_{d b}, \boldsymbol{\alpha}\right)}{\partial a_{3, d a v}}=\frac{a_{0 d a v} T_{d b}^{3.5}}{\left(a_{1 d a v}+a_{2 d a v} T_{d b}+a_{3 d a v} T_{d b}^{2}\right)^{2}} .
$$

The derivatives of the "liquid continuity equations" [cf. Equations (A1)-(A3)] with respect to the parameter $\alpha^{(22)} \equiv a_{0 f}$ are as follows:

$$
\frac{\partial N_{1}^{(i)}}{\partial \alpha^{(22)}}=\frac{\partial N_{1}^{(i)}}{\partial a_{0 f}} \equiv a_{1}^{i, 22}=0 ; \quad \ell=1 ; i=1, \ldots, I ; j=22 .
$$

The derivatives of the "liquid continuity equations" [cf. Equations (A1)-(A3)] with respect to the parameter $\alpha^{(23)} \equiv a_{1 f}$ are as follows:

$$
\frac{\partial N_{1}^{(i)}}{\partial \alpha^{(23)}}=\frac{\partial N_{1}^{(i)}}{\partial a_{1 f}} \equiv a_{1}^{i, 23}=0 ; \quad \ell=1 ; i=1, \ldots, I ; j=23 .
$$

The derivatives of the "liquid continuity equations" [cf. Equations (A1)-(A3)] with respect to the parameter $\alpha^{(24)} \equiv a_{0 g}$ are as follows:

$$
\frac{\partial N_{1}^{(i)}}{\partial \alpha^{(24)}}=\frac{\partial N_{1}^{(i)}}{\partial a_{0 g}} \equiv a_{1}^{i, 24}=0 ; \quad \ell=1 ; i=1, \ldots, I ; j=24 .
$$

The derivatives of the "liquid continuity equations" [cf. Equations (A1)-(A3)] with respect to the parameter $\alpha^{(25)} \equiv a_{1 g}$ are as follows:

$$
\frac{\partial N_{1}^{(i)}}{\partial \alpha^{(25)}}=\frac{\partial N_{1}^{(i)}}{\partial a_{1 g}} \equiv a_{1}^{i, 25}=0 ; \quad \ell=1 ; i=1, \ldots, I ; j=25 .
$$

The derivatives of the "liquid continuity equations" [cf. Equations (A1)-(A3)] with respect to the parameter $\alpha^{(26)} \equiv a_{0, N u}$ are as follows:

$$
\begin{gathered}
\frac{\partial N_{1}^{(i)}}{\partial \alpha^{(26)}}=\frac{\partial N_{1}^{(i)}}{\partial a_{0, N u}} \equiv a_{1}^{i, 26}=\frac{1}{\bar{R}}\left[\frac{P_{v s}^{(i+1)}\left(T_{w}^{(i+1)}, \boldsymbol{\alpha}\right)}{T_{w}^{(i+1)}}-\frac{\omega^{(i)} P_{a t m}}{\left(0.622+\omega^{(i)}\right) T_{a}^{(i)}}\right] \frac{\partial M\left(m_{a}, \boldsymbol{\alpha}\right)}{\partial N u(\operatorname{Re}, \boldsymbol{\alpha})} \frac{\partial N u(\operatorname{Re}, \boldsymbol{\alpha})}{\partial a_{0, N u}} ; \\
\ell=1 ; i=1, \ldots, I ; j=26
\end{gathered}
$$

where $\frac{\partial M\left(m_{a}, \alpha\right)}{\partial N u(\operatorname{Re}, \alpha)}$ was defined previously in Equation (A23), and

$$
\frac{\partial N u(\operatorname{Re}, \alpha)}{\partial a_{0, N u}}=\left\{\begin{array}{cc}
1 & \operatorname{Re}_{d}<2300 \\
0 & 2300 \leq \operatorname{Re}_{d} \leq 10000 \\
0 & \operatorname{Re}_{d}>10000
\end{array}\right.
$$


The derivatives of the "liquid continuity equations" [cf. Equations (A1)-(A3)] with respect to the parameter $\alpha^{(27)} \equiv a_{1, N u}$ are as follows:

$$
\begin{gathered}
\frac{\partial N_{1}^{(i)}}{\partial \alpha^{(27)}}=\frac{\partial N_{1}^{(i)}}{\partial a_{1, N u}} \equiv a_{1}^{i, 27}=\frac{1}{\bar{R}}\left[\frac{P_{v s}^{(i+1)}\left(T_{w}^{(i+1)}, \boldsymbol{\alpha}\right)}{T_{w}^{(i+1)}}-\frac{\omega^{(i)} P_{a t m}}{\left(0.622+\omega^{(i)}\right) T_{a}^{(i)}}\right] \frac{\partial M\left(m_{a}, \boldsymbol{\alpha}\right)}{\partial N u(\operatorname{Re}, \boldsymbol{\alpha})} \frac{\partial N u(\operatorname{Re}, \boldsymbol{\alpha})}{\partial a_{1, N u}} ; \\
\ell=1 ; i=1, \ldots, I ; j=27,
\end{gathered}
$$

where $\frac{\partial M\left(m_{a}, \alpha\right)}{\partial N u(\operatorname{Re}, \alpha)}$ was defined previously in Equation (A23), and:

$$
\frac{\partial N u(\operatorname{Re}, \alpha)}{\partial a_{1, N u}}=\left\{\begin{array}{cc}
0 & \operatorname{Re}_{d}<2300 \\
\operatorname{Re}\left(m_{a}, \boldsymbol{\alpha}\right) & 2300 \leq \operatorname{Re}_{d} \leq 10000 \\
0 & \operatorname{Re}_{d}>10000
\end{array}\right.
$$

The derivatives of the "liquid continuity equations" [cf. Equations (A1)-(A3)] with respect to the parameter $\alpha^{(28)} \equiv a_{2, \mathrm{~N} u}$ are as follows:

$$
\begin{gathered}
\frac{\partial N_{1}^{(i)}}{\partial \alpha^{(28)}}=\frac{\partial N_{1}^{(i)}}{\partial a_{2, N u}} \equiv a_{1}^{i, 28}=\frac{1}{\bar{R}}\left[\frac{P_{v s}^{(i+1)}\left(T_{w}^{(i+1)}, \boldsymbol{\alpha}\right)}{T_{w}^{(i+1)}}-\frac{\omega^{(i)} P_{a t m}}{\left(0.622+\omega^{(i)}\right) T_{a}^{(i)}}\right] \frac{\partial M\left(m_{a}, \boldsymbol{\alpha}\right)}{\partial N u(\operatorname{Re}, \boldsymbol{\alpha})} \frac{\partial N u(\operatorname{Re}, \boldsymbol{\alpha})}{\partial a_{2, N u}} ; \\
\ell=1 ; i=1, \ldots, I ; j=28
\end{gathered}
$$

where $\frac{\partial M\left(m_{a}, \boldsymbol{\alpha}\right)}{\partial N u(\operatorname{Re}, \boldsymbol{\alpha})}$ was defined previously in Equation (A23), and:

$$
\frac{\partial N u(\operatorname{Re}, \boldsymbol{\alpha})}{\partial a_{2, N u}}=\left\{\begin{array}{cc}
0 & \operatorname{Re}_{d}<2300 \\
1 & 2300 \leq \operatorname{Re}_{d} \leq 10000 \\
0 & \operatorname{Re}_{d}>10000
\end{array}\right.
$$

The derivatives of the "liquid continuity equations" [cf. Equations (A1)-(A3)] with respect to the parameter $\alpha^{(29)} \equiv a_{3, \mathrm{Nu}}$ are as follows:

$$
\begin{gathered}
\frac{\partial N_{1}^{(i)}}{\partial \alpha^{(29)}}=\frac{\partial N_{1}^{(i)}}{\partial a_{3, N u}} \equiv a_{1}^{i, 29}=\frac{1}{\bar{R}}\left[\frac{P_{v s}^{(i+1)}\left(T_{w}^{(i+1)}, \boldsymbol{\alpha}\right)}{T_{w}^{(i+1)}}-\frac{\omega^{(i)} P_{a t m}}{\left(0.622+\omega^{(i)}\right) T_{a}^{(i)}}\right] \frac{\partial M\left(m_{a}, \boldsymbol{\alpha}\right)}{\partial N u(\operatorname{Re}, \boldsymbol{\alpha})} \frac{\partial N u(\operatorname{Re}, \boldsymbol{\alpha})}{\partial a_{3, N u}} \\
\quad \ell=1 ; i=1, \ldots, I ; j=29,
\end{gathered}
$$

where $\frac{\partial M\left(m_{a}, \boldsymbol{\alpha}\right)}{\partial N u(\operatorname{Re}, \boldsymbol{\alpha})}$ was defined previously in Equation (A23), and:

$$
\frac{\partial N u(\operatorname{Re}, \boldsymbol{\alpha})}{\partial a_{3, N u}}= \begin{cases}0 & \operatorname{Re}_{d}<2300 \\ 0 & 2300 \leq \operatorname{Re}_{d} \leq 10000 \\ {\left[\operatorname{Re}\left(m_{a}, \boldsymbol{\alpha}\right)\right]^{0.8} \cdot \operatorname{Pr}^{\frac{1}{3}}} & \operatorname{Re}_{d}>10000\end{cases}
$$

The derivatives of the "liquid continuity equations" [cf. Equations (A1)-(A3)] with respect to the parameter $\alpha^{(30)} \equiv W_{d k x}$ are as follows:

$$
\frac{\partial N_{1}^{(i)}}{\partial \alpha^{(30)}}=\frac{\partial N_{1}^{(i)}}{\partial W_{d k x}} \equiv a_{1}^{i, 30}=0 ; \quad \ell=1 ; i=1, \ldots, I ; j=30 .
$$

The derivatives of the "liquid continuity equations" [cf. Equations (A1)-(A3)] with respect to the parameter $\alpha^{(31)} \equiv W_{d k y}$ are as follows:

$$
\frac{\partial N_{1}^{(i)}}{\partial \alpha^{(31)}}=\frac{\partial N_{1}^{(i)}}{\partial W_{d k y}} \equiv a_{1}^{i, 31}=0 ; \quad \ell=1 ; i=1, \ldots, I ; j=31 .
$$


The derivatives of the "liquid continuity equations" [cf. Equations (A1)-(A3)] with respect to the parameter $\alpha^{(32)} \equiv \Delta z_{d k}$ are as follows:

$$
\frac{\partial N_{1}^{(i)}}{\partial \alpha^{(32)}}=\frac{\partial N_{1}^{(i)}}{\partial \Delta z_{d k}} \equiv a_{1}^{i, 32}=0 ; \quad \ell=1 ; i=1, \ldots, I ; j=32 .
$$

The derivatives of the "liquid continuity equations" [cf. Equations (A1)-(A3)] with respect to the parameter $\alpha^{(33)} \equiv \Delta z_{\text {fan }}$ are as follows:

$$
\frac{\partial N_{1}^{(i)}}{\partial \alpha^{(33)}}=\frac{\partial N_{1}^{(i)}}{\partial \Delta z_{f a n}} \equiv a_{1}^{i, 33}=0 ; \quad \ell=1 ; i=1, \ldots, I ; j=33 .
$$

The derivatives of the "liquid continuity equations" [cf. Equations (A1)-(A3)] with respect to the parameter $\alpha^{(34)} \equiv D_{\text {fan }}$ are as follows:

$$
\begin{gathered}
\frac{\partial N_{1}^{(i)}}{\partial \alpha^{(34)}}=\frac{\partial N_{1}^{(i)}}{\partial D_{f a n}} \equiv a_{1}^{i, 34}=\frac{1}{\bar{R}}\left[\frac{P_{v s}^{(i+1)}\left(T_{w}^{(i+1)}, \boldsymbol{\alpha}\right)}{T_{w}^{(i+1)}}-\frac{\omega^{(i)} P_{a t m}}{\left(0.622+\omega^{(i)}\right) T_{a}^{(i)}}\right] \frac{\partial M\left(m_{a}, \boldsymbol{\alpha}\right)}{\partial N u(\operatorname{Re}, \boldsymbol{\alpha})} \frac{\partial N u(\operatorname{Re}, \boldsymbol{\alpha})}{\partial m_{a}} \frac{\partial m_{a}}{\partial D_{f a n}} \\
\ell=1 ; i=1, \ldots, I ; j=34
\end{gathered}
$$

where $\frac{\partial M\left(m_{a}, \boldsymbol{\alpha}\right)}{\partial N u(\operatorname{Re}, \boldsymbol{\alpha})}$ and $\frac{\partial N u(\operatorname{Re}, \boldsymbol{\alpha})}{\partial m_{a}}$ were defined previously in Equations (A23) and (A24), respectively, and:

$$
\frac{\partial m_{a}}{\partial D_{f a n}}=\frac{2 \cdot m_{a}}{D_{f a n}}
$$

The derivatives of the "liquid continuity equations" [cf. Equations (A1)-(A3)] with respect to the parameter $\alpha^{(35)} \equiv \Delta z_{\text {fill }}$ are as follows:

$$
\frac{\partial N_{1}^{(i)}}{\partial \alpha^{(35)}}=\frac{\partial N_{1}^{(i)}}{\partial \Delta z_{f i l l}} \equiv a_{1}^{i, 35}=0 ; \quad \ell=1 ; i=1, \ldots, I ; j=35 .
$$

The derivatives of the "liquid continuity equations" [cf. Equations (A1)-(A3)] with respect to the parameter $\alpha^{(36)} \equiv \Delta z_{\text {rain }}$ are as follows:

$$
\frac{\partial N_{1}^{(i)}}{\partial \alpha^{(36)}}=\frac{\partial N_{1}^{(i)}}{\partial \Delta z_{\text {rain }}} \equiv a_{1}^{i, 36}=0 ; \quad \ell=1 ; i=1, \ldots, I ; j=36 .
$$

The derivatives of the "liquid continuity equations" [cf. Equations (A1)-(A3)] with respect to the parameter $\alpha^{(37)} \equiv \Delta z_{b s}$ are as follows:

$$
\frac{\partial N_{1}^{(i)}}{\partial \alpha^{(37)}}=\frac{\partial N_{1}^{(i)}}{\partial \Delta z_{b s}} \equiv a_{1}^{i, 37}=0 ; \quad \ell=1 ; i=1, \ldots, I ; j=37 .
$$

The derivatives of the "liquid continuity equations" [cf. Equations (A1)-(A3)] with respect to the parameter $\alpha^{(38)} \equiv \Delta z_{d e}$ are as follows:

$$
\frac{\partial N_{1}^{(i)}}{\partial \alpha^{(38)}}=\frac{\partial N_{1}^{(i)}}{\partial \Delta z_{d e}} \equiv a_{1}^{i, 38}=0 ; \quad \ell=1 ; i=1, \ldots, I ; j=38 .
$$

The derivatives of the "liquid continuity equations" [cf. Equations (A1)-(A3)] with respect to the parameter $\alpha^{(39)} \equiv D_{h}$ are as follows:

$$
\begin{gathered}
\frac{\partial N_{1}^{(i)}}{\partial \alpha^{(39)}}=\frac{\partial N_{1}^{(i)}}{\partial D_{h}} \equiv a_{1}^{i, 39}=\frac{1}{\bar{R}}\left[\frac{P_{v s}^{(i+1)}\left(T_{w}^{(i+1)}, \alpha\right)}{T_{w}^{(i+1)}}-\frac{\omega^{(i)} P_{a t m}}{\left(0.622+\omega^{(i)}\right) T_{a}^{(i)}}\right] \frac{\partial M\left(m_{a}, \boldsymbol{\alpha}\right)}{\partial D_{h}} ; \\
\ell=1 ; i=1, \ldots, I ; j=39
\end{gathered}
$$


where:

$$
\frac{\partial M\left(m_{a}, \boldsymbol{\alpha}\right)}{\partial D_{h}}= \begin{cases}-M\left(m_{a}, \boldsymbol{\alpha}\right) / D_{h} & \operatorname{Re}_{d}<2300 \\ -\frac{a_{2, N u} M\left(m_{a}, \boldsymbol{\alpha}\right)}{N u(\operatorname{Re}, \boldsymbol{\alpha}) D_{h}} & 2300 \leq \operatorname{Re}_{d} \leq 10000 \\ -0.2 \cdot M\left(m_{a}, \boldsymbol{\alpha}\right) / D_{h} & \operatorname{Re}_{d}>10000\end{cases}
$$

The derivatives of the "liquid continuity equations" [cf. Equations (A1)-(A3)] with respect to the parameter $\alpha^{(40)} \equiv A_{\text {fill }}$ are as follows:

$$
\begin{gathered}
\frac{\partial N_{1}^{(i)}}{\partial \alpha^{(40)}}=\frac{\partial N_{1}^{(i)}}{\partial A_{\text {fill }}} \equiv a_{1}^{i, 40}=\frac{1}{R}\left[\frac{P_{v s}^{(i+1)}\left(T_{w}^{(i+1)}, \alpha\right)}{T_{w}^{(i+1)}}-\frac{\omega^{(i)} P_{a t m}}{\left(0.622+\omega^{(i)}\right) T_{a}^{(i)}}\right] \frac{\partial M\left(m_{a}, \alpha\right)}{\partial A_{f i l l}} ; \\
\ell=1 ; i=1, \ldots, I ; j=40
\end{gathered}
$$

where:

$$
\frac{\partial M\left(m_{a}, \boldsymbol{\alpha}\right)}{\partial A_{\text {fill }}}= \begin{cases}0 & \operatorname{Re}_{d}<2300 \\ -\frac{a_{1, N u} M\left(m_{a}, \boldsymbol{\alpha}\right) \operatorname{Re}\left(m_{a}, \boldsymbol{\alpha}\right)}{N u(\operatorname{Re}, \boldsymbol{\alpha}) A_{\text {fill }}} & 2300 \leq \operatorname{Re}_{d} \leq 10000 \\ -0.8 \cdot M\left(m_{a}, \boldsymbol{\alpha}\right) / A_{\text {fill }} & \operatorname{Re}_{d}>10000\end{cases}
$$

The derivatives of the "liquid continuity equations" [cf. Equations (A1)-(A3)] with respect to the parameter $\alpha^{(41)} \equiv A_{\text {surf }}$ are as follows:

$$
\begin{gathered}
\frac{\partial N_{1}^{(i)}}{\partial \alpha^{(41)}}=\frac{\partial N_{1}^{(i)}}{\partial A_{\text {surf }}} \equiv a_{1}^{i, 41}=\frac{1}{\bar{R}}\left[\frac{P_{v s}^{(i+1)}\left(T_{w}^{(i+1)}, \boldsymbol{\alpha}\right)}{T_{w}^{(i+1)}}-\frac{\omega^{(i)} P_{a t m}}{\left(0.622+\omega^{(i)}\right) T_{a}^{(i)}}\right] \frac{\partial M\left(m_{a}, \boldsymbol{\alpha}\right)}{\partial A_{\text {surf }}} \\
\ell=1 ; i=1, \ldots, I ; j=41
\end{gathered}
$$

where:

$$
\frac{\partial M\left(m_{a}, \boldsymbol{\alpha}\right)}{\partial A_{\text {surf }}}=\frac{M\left(m_{a}, \boldsymbol{\alpha}\right)}{A_{\text {surf }}}
$$

The derivatives of the "liquid continuity equations" [cf. Equations (A1)-(A3)] with respect to the parameter $\alpha^{(42)} \equiv \operatorname{Pr}$ are as follows:

$$
\begin{gathered}
\frac{\partial N_{1}^{(i)}}{\partial \alpha^{(42)}}=\frac{\partial N_{1}^{(i)}}{\partial \operatorname{Pr}} \equiv a_{1}^{i, 42}=\frac{1}{\bar{R}}\left[\frac{P_{v s}^{(i+1)}\left(T_{w}^{(i+1)}, \alpha\right)}{T_{w w}^{(i+1)}}-\frac{\omega^{(i)} P_{a t m}}{\left(0.622+\omega^{(i)}\right) T_{a}^{(i)}}\right] \frac{\partial M\left(m_{a}, \alpha\right)}{\partial \operatorname{Pr}} ; \\
\ell=1 ; i=1, \ldots, I ; j=42,
\end{gathered}
$$

where:

$$
\frac{\partial M\left(m_{a}, \boldsymbol{\alpha}\right)}{\partial \operatorname{Pr}}=\left\{\begin{array}{ll}
-M\left(m_{a}, \boldsymbol{\alpha}\right) /(3 \cdot \operatorname{Pr}) & \operatorname{Re}_{d} \leq 10000 \\
0 & \operatorname{Re}_{d}>10000
\end{array} .\right.
$$

The derivatives of the "liquid continuity equations" [cf. Equations (A1)-(A3)] with respect to the parameter $\alpha^{(43)} \equiv V_{w}$ are as follows:

$$
\frac{\partial N_{1}^{(i)}}{\partial \alpha^{(43)}}=\frac{\partial N_{1}^{(i)}}{\partial V_{w}} \equiv a_{1}^{i, 43}=0 ; \quad \ell=1 ; i=1, \ldots, I ; j=43 .
$$

The derivatives of the "liquid continuity equations" [cf. Equations (A1)-(A3)] with respect to the parameter $\alpha^{(44)} \equiv V_{\text {exit }}$ are as follows:

$$
\begin{gathered}
\frac{\partial N_{1}^{(i)}}{\partial \alpha^{(44)}}=\frac{\partial N_{1}^{(i)}}{\partial V_{e x i t}} \equiv a_{1}^{i, 44}=\frac{1}{\bar{R}}\left[\frac{P_{v s}^{(i+1)}\left(T_{w}^{(i+1)}, \alpha\right)}{T_{w v}^{(i+1)}}-\frac{\omega^{(i)} P_{a t m}}{\left(0.622+\omega^{(i)}\right) T_{a}^{(i)}}\right] \frac{\partial M\left(m_{a}, \boldsymbol{\alpha}\right)}{\partial N u(\operatorname{Re}, \boldsymbol{\alpha})} \frac{\partial N u(\operatorname{Re}, \boldsymbol{\alpha})}{\partial m_{a}} \frac{\partial m_{a}}{\partial V_{e x i t}} \\
\ell=1 ; i=1, \ldots, I ; j=44
\end{gathered}
$$


where $\frac{\partial M\left(m_{a}, \boldsymbol{\alpha}\right)}{\partial N u(\operatorname{Re}, \boldsymbol{\alpha})}$ and $\frac{\partial N u(\operatorname{Re}, \boldsymbol{\alpha})}{\partial m_{a}}$ were defined previously in Equations (A23) and (A24), respectively, and

$$
\frac{\partial m_{a}}{\partial V_{\text {exit }}}=\frac{P_{a t m}}{R_{a i r} T_{a, i n}} \cdot \frac{\pi D_{f a n}{ }^{2}}{4}
$$

The derivatives of the "liquid continuity equations" [cf. Equations (A1)-(A3)] with respect to the parameter $\alpha^{(45)} \equiv m_{w, \text { in }}$ are as follows:

$$
\begin{gathered}
\frac{\partial N_{1}^{(1)}}{\partial \alpha^{(45)}}=\frac{\partial N_{1}^{(1)}}{\partial m_{w, i n}} \equiv a_{1}^{1,45}=-1 ; \quad \ell=1 ; i=1 ; j=45, \\
\frac{\partial N_{1}^{(i)}}{\partial \alpha^{(45)}}=\frac{\partial N_{1}^{(i)}}{\partial m_{w, i n}} \equiv a_{1}^{i, 45}=0 ; \quad \ell=1 ; i=2, \ldots, I ; j=45 .
\end{gathered}
$$

The derivatives of the "liquid continuity equations" [cf. Equations (A1)-(A3)] with respect to the parameter $\alpha^{(46)} \equiv T_{a, i n}$ are as follows:

$$
\frac{\partial N_{1}^{(i)}}{\partial \alpha^{(46)}}=\frac{\partial N_{1}^{(i)}}{\partial T_{a, i n}} \equiv a_{1}^{i, 46}=0 ; \quad \ell=1 ; i=1, \ldots, I ; j=46 .
$$

The derivatives of the "liquid continuity equations" [cf. Equations (A1)-(A3)] with respect to the parameter $\alpha^{(47)} \equiv m_{a}$ are as follows:

$$
\begin{gathered}
\frac{\partial N_{1}^{(i)}}{\partial \alpha^{(47)}}=\frac{\partial N_{1}^{(i)}}{\partial m_{a}} \equiv a_{1}^{i, 47}=\frac{1}{\bar{R}}\left[\frac{P_{v s}^{(i+1)}\left(T_{w}^{(i+1)}, \boldsymbol{\alpha}\right)}{T_{w}^{(i+1)}}-\frac{\omega^{(i)} P_{a t m}}{\left(0.622+\omega^{(i)}\right) T_{a}^{(i)}}\right] \frac{\partial M\left(m_{a}, \alpha\right)}{\partial N u(\operatorname{Re}, \boldsymbol{\alpha})} \frac{\partial N u(\operatorname{Re}, \boldsymbol{\alpha})}{\partial m_{a}} ; \\
\ell=1 ; i=1, \ldots, I ; j=47,
\end{gathered}
$$

where $\frac{\partial M\left(m_{a}, \boldsymbol{\alpha}\right)}{\partial N u(\operatorname{Re}, \boldsymbol{\alpha})}$ and $\frac{\partial N u(\operatorname{Re}, \boldsymbol{\alpha})}{\partial m_{a}}$ were defined previously in Equations (A23) and (A24), respectively.

The derivatives of the "liquid continuity equations" [cf. Equations (A1)-(A3)] with respect to the parameter $\alpha^{(48)} \equiv \omega_{\text {in }}$ are as follows:

$$
\frac{\partial N_{1}^{(i)}}{\partial \alpha^{(48)}}=\frac{\partial N_{1}^{(i)}}{\partial \omega_{\text {in }}} \equiv a_{1}^{i, 48}=0 ; \quad \ell=1 ; i=1, \ldots, I ; j=48 .
$$

The derivatives of the "liquid continuity equations" [cf. Equations (A1)-(A3)] with respect to the parameter $\alpha^{(49)} \equiv \operatorname{Re}_{d}$ are as follows:

$$
\begin{gathered}
\frac{\partial N_{1}^{(i)}}{\partial \alpha^{(49)}}=\frac{\partial N_{1}^{(i)}}{\partial \operatorname{Re}_{d}} \equiv a_{1}^{i, 49}=\frac{1}{\bar{R}}\left[\frac{P_{v s}^{(i+1)}\left(T_{w}^{(i+1)}, \boldsymbol{\alpha}\right)}{T_{w}^{(i+1)}}-\frac{\omega^{(i)} P_{a t m}}{\left(0.622+\omega^{(i)}\right) T_{a}^{(i)}}\right] \frac{\partial M\left(m_{a}, \boldsymbol{\alpha}\right)}{\partial N u\left(\operatorname{Re}_{d}, \boldsymbol{\alpha}\right)} \frac{\partial N u\left(\operatorname{Re}_{d}, \boldsymbol{\alpha}\right)}{\partial \operatorname{Re}_{d}} ; \\
\ell=1 ; i=1, \ldots, I ; j=49,
\end{gathered}
$$

where $\frac{\partial M\left(m_{a}, \boldsymbol{\alpha}\right)}{\partial N u(\operatorname{Re}, \boldsymbol{\alpha})}$ was defined in Equation (A23), and:

$$
\frac{\partial N u\left(\operatorname{Re}_{d}, \boldsymbol{\alpha}\right)}{\partial \operatorname{Re}_{d}}= \begin{cases}0 & \operatorname{Re}_{d}<2300 \\ a_{1, N u} & 2300 \leq \operatorname{Re}_{d} \leq 10000 \\ 0.8 \cdot a_{3, N u} \cdot \operatorname{Re}_{d}{ }^{-0.2} \operatorname{Pr}^{1 / 3} & \operatorname{Re}_{d}>10000\end{cases}
$$

The derivatives of the "liquid continuity equations" [cf. Equations (A1)-(A3)] with respect to the parameter $\alpha^{(50)} \equiv S c$ are as follows:

$$
\begin{gathered}
\frac{\partial N_{1}^{(i)}}{\partial \alpha^{(50)}}=\frac{\partial N_{1}^{(i)}}{\partial S c} \equiv a_{1}^{i, 50}=\frac{1}{\bar{R}}\left[\frac{P_{v s}^{(i+1)}\left(T_{w}^{(i+1)}, \alpha\right)}{T_{w}^{(i+1)}}-\frac{\omega^{(i)} P_{a t m}}{\left(0.622+\omega^{(i)}\right) T_{a}^{(i)}}\right] \frac{\partial M\left(m_{a}, \alpha\right)}{\partial S c} ; \\
\ell=1 ; i=1, \ldots, I ; j=50,
\end{gathered}
$$


where:

$$
\frac{\partial M\left(m_{a}, \boldsymbol{\alpha}\right)}{\partial S c}=\frac{1}{3} \frac{M\left(m_{a}, \boldsymbol{\alpha}\right)}{S c} .
$$

The derivatives of the "liquid continuity equations" [cf. Equations (A1)-(A3)] with respect to the parameter $\alpha^{(51)} \equiv S h$ are as follows:

$$
\begin{gathered}
\frac{\partial N_{1}^{(i)}}{\partial \alpha^{(51)}}=\frac{\partial N_{1}^{(i)}}{\partial S h} \equiv a_{1}^{i, 51}=\frac{1}{\bar{R}}\left[\frac{P_{v s}^{(i+1)}\left(T_{w}^{(i+1)}, \alpha\right)}{T_{w}^{(i+1)}}-\frac{\omega^{(i)} P_{a t m}}{\left(0.622+\omega^{(i)}\right) T_{a}^{(i)}}\right] \frac{\partial M\left(m_{a}, \alpha\right)}{\partial S h} ; \\
\ell=1 ; i=1, \ldots, I ; j=51,
\end{gathered}
$$

where:

$$
\frac{\partial M\left(m_{a}, \boldsymbol{\alpha}\right)}{\partial S h}=\frac{M\left(m_{a}, \boldsymbol{\alpha}\right)}{S h} .
$$

The derivatives of the "liquid continuity equations" [cf. Equations (A1)-(A3)] with respect to the parameter $\alpha^{(52)} \equiv N u$ are as follows:

$$
\begin{gathered}
\frac{\partial N_{1}^{(i)}}{\partial \alpha^{(52)}}=\frac{\partial N_{1}^{(i)}}{\partial N u} \equiv a_{1}^{i, 52}=\frac{1}{\bar{R}}\left[\frac{P_{v s}^{(i+1)}\left(T_{w}^{(i+1)}, \boldsymbol{\alpha}\right)}{T_{w}^{(i+1)}}-\frac{\omega^{(i)} P_{a t m}}{\left(0.622+\omega^{(i)}\right) T_{a}^{(i)}}\right] \frac{\partial M\left(m_{a,}, \boldsymbol{\alpha}\right)}{\partial N u} ; \\
\ell=1 ; i=1, \ldots, I ; j=52,
\end{gathered}
$$

where $\frac{\partial M\left(m_{a}, \boldsymbol{\alpha}\right)}{\partial N u(\operatorname{Re}, \boldsymbol{\alpha})}$ was defined in Equation (A23).

\section{A2. Derivatives of the Liquid Energy Balance Equations with Respect to the Parameters}

The derivatives of the liquid energy balance equations [cf. Equations (A4)-(A6)] with respect to the parameter $\alpha^{(1)} \equiv T_{d b}$ are as follows:

$$
\frac{\partial N_{2}^{(i)}}{\partial \alpha^{(1)}}=\frac{\partial N_{2}^{(i)}}{\partial T_{d b}} \equiv a_{2}^{i, 1}=0 ; \quad \ell=2 ; i=1, \ldots, I ; j=1 .
$$

The derivatives of the liquid energy balance equations [cf. Equations (A4)-(A6)] with respect to the parameter $\alpha^{(2)} \equiv T_{d p}$ are as follows:

$$
\frac{\partial N_{2}^{(i)}}{\partial \alpha^{(2)}}=\frac{\partial N_{2}^{(i)}}{\partial T_{d p}} \equiv a_{2}^{i, 2}=0 ; \quad \ell=2 ; i=1, \ldots, I ; j=2 .
$$

The derivatives of the liquid energy balance equations [cf. Equations (A4)-(A6)] with respect to the parameter $\alpha^{(3)} \equiv T_{w, i n}$ are as follows:

$$
\begin{gathered}
\frac{\partial N_{2}^{(1)}}{\partial \alpha^{(3)}}=\frac{\partial N_{2}^{(1)}}{\partial T_{w, i n}} \equiv a_{2}^{1,3}=m_{w, i n} \frac{\partial h_{f}^{(1)}\left(T_{w, i n}, \boldsymbol{\alpha}\right)}{\partial T_{w, i n}}+h_{f}^{(1)}\left(T_{w, i n}, \boldsymbol{\alpha}\right) \frac{\partial m_{w, i n}}{\partial T_{w, i n}}-h_{g, w}^{(2)}\left(T_{w}^{(2)}, \boldsymbol{\alpha}\right) \frac{\partial m_{w, i n}}{\partial T_{w, i n}} ; \\
\ell=2 ; i=1 ; j=3,
\end{gathered}
$$

where $\frac{\partial m_{w, i n}}{\partial T_{w, i n}}$ was defined in Equation (A20), and:

$$
\begin{gathered}
\frac{\partial h_{f}^{(1)}\left(T_{w, i n}, \alpha\right)}{\partial T_{w, i n}}=a_{1 f}, \\
\frac{\partial N_{2}^{(i)}}{\partial \alpha^{(3)}}=\frac{\partial N_{2}^{(i)}}{\partial T_{w, i n}} \equiv a_{2}^{i, 3}=0 ; \quad \ell=2 ; i=2, \ldots I ; j=3 .
\end{gathered}
$$


The derivatives of the liquid energy balance equations [cf. Equations (A4)-(A6)] with respect to the parameter $\alpha^{(4)} \equiv P_{\text {atm }}$ are as follows:

$$
\begin{gathered}
\frac{\partial N_{2}^{(i)}}{\partial \alpha^{(4)}}=\frac{\partial N_{2}^{(i)}}{\partial P_{a t m}} \equiv a_{2}^{i, 4}=-\left(T_{w}^{(i+1)}-T_{a}^{(i)}\right) \frac{\partial H\left(m_{a}, \alpha\right)}{\partial N u(\operatorname{Re}, \boldsymbol{\alpha})} \frac{\partial N u(\operatorname{Re}, \boldsymbol{\alpha})}{\partial m_{a}} \frac{\partial m_{a}}{\partial P_{a t m}} ; \\
\ell=2 ; i=1, \ldots, I ; j=4,
\end{gathered}
$$

where $\frac{\partial N u(R e, \alpha)}{\partial m_{a}}$ and $\frac{\partial m_{a}}{\partial P_{a t m}}$ were defined in Equations (A24) and (A25), respectively, and:

$$
\frac{\partial H\left(m_{a}, \boldsymbol{\alpha}\right)}{\partial N u(\operatorname{Re}, \boldsymbol{\alpha})}=\frac{H\left(m_{a}, \boldsymbol{\alpha}\right)}{N u(\operatorname{Re}, \boldsymbol{\alpha})} .
$$

The derivatives of the liquid energy balance equations [cf. Equations (A4)-(A6)] with respect to the parameter $\alpha^{(5)} \equiv w_{\text {tas }}$ are as follows:

$$
\frac{\partial N_{2}^{(i)}}{\partial \alpha^{(5)}}=\frac{\partial N_{2}^{(i)}}{\partial w_{t s a}} \equiv a_{2}^{i, 5}=-\left(T_{w}^{(i+1)}-T_{a}^{(i)}\right) \frac{\partial H\left(m_{a}, \alpha\right)}{\partial w_{t s a}} ; \quad \ell=2 ; i=1, \ldots, I ; j=5,
$$

where:

$$
\frac{\partial H\left(m_{a}, \boldsymbol{\alpha}\right)}{\partial w_{t s a}}=\frac{f_{h t} N u(\operatorname{Re}, \boldsymbol{\alpha}) k_{\text {air }} A_{\text {surf }}}{\mathrm{D}_{h} I} .
$$

The derivatives of the liquid energy balance equations [cf. Equations (A4)-(A6)] with respect to the parameter $\alpha^{(6)} \equiv k_{\text {sum }}$ are as follows:

$$
\frac{\partial N_{2}^{(i)}}{\partial \alpha^{(6)}}=\frac{\partial N_{2}^{(i)}}{\partial k_{\text {sum }}} \equiv a_{2}^{i, 6}=0 ; \quad \ell=2 ; i=1, \ldots, I ; j=6 .
$$

The derivatives of the liquid energy balance equations [cf. Equations (A4)-(A6)] with respect to the parameter $\alpha^{(7)} \equiv \mu$ are as follows:

$$
\frac{\partial N_{2}^{(i)}}{\partial \alpha^{(7)}}=\frac{\partial N_{2}^{(i)}}{\partial \mu} \equiv a_{2}^{i, 7}=-\left(T_{w}^{(i+1)}-T_{a}^{(i)}\right) \frac{\partial H\left(m_{a}, \alpha\right)}{\partial \mu} ; \quad \ell=2 ; i=1, \ldots, I ; j=7,
$$

where:

$$
\frac{\partial H\left(m_{a}, \boldsymbol{\alpha}\right)}{\partial \mu}= \begin{cases}0 & \operatorname{Re}_{d}<2300 \\ -\frac{a_{1, N u} \cdot H\left(m_{a}, \boldsymbol{\alpha}\right) \cdot \operatorname{Re}\left(m_{a}, \boldsymbol{\alpha}\right)}{N u(\operatorname{Re}, \boldsymbol{\alpha}) \cdot \mu} & 2300 \leq \operatorname{Re}_{d} \leq 10000 \\ -0.8 \cdot \frac{H\left(m_{a}, \alpha\right)}{\mu} & \operatorname{Re}_{d}>10000\end{cases}
$$

The derivatives of the liquid energy balance equations [cf. Equations (A4)-(A6)] with respect to the parameter $\alpha^{(8)} \equiv v$ are as follows:

$$
\frac{\partial N_{2}^{(i)}}{\partial \alpha^{(8)}}=\frac{\partial N_{2}^{(i)}}{\partial v} \equiv a_{2}^{i, 8}=0 ; \quad \ell=2 ; i=1, \ldots, I ; j=8 .
$$

The derivatives of the liquid energy balance equations [cf. Equations (A4)-(A6)] with respect to the parameter $\alpha^{(9)} \equiv k_{\text {air }}$ are as follows:

$$
\frac{\partial N_{2}^{(i)}}{\partial \alpha^{(9)}}=\frac{\partial N_{2}^{(i)}}{\partial k_{\text {air }}} \equiv a_{2}^{i, 9}=-\left(T_{w}^{(i+1)}-T_{a}^{(i)}\right) \frac{\partial H\left(m_{a}, \alpha\right)}{\partial k_{a i r}} ; \quad \ell=2 ; i=1, \ldots, I ; j=9,
$$

where:

$$
\frac{\partial H\left(m_{a}, \boldsymbol{\alpha}\right)}{\partial k_{\text {air }}}=\frac{H\left(m_{a}, \boldsymbol{\alpha}\right)}{k_{\text {air }}}=\frac{f_{h t} N u(\operatorname{Re}, \boldsymbol{\alpha}) w_{\text {tsa }} A_{\text {surf }}}{\mathrm{D}_{h} I}
$$


The derivatives of the liquid energy balance equations [cf. Equations (A4)-(A6)] with respect to the parameter $\alpha^{(10)} \equiv f_{h t}$ are as follows:

$$
\frac{\partial N_{2}^{(i)}}{\partial \alpha^{(10)}}=\frac{\partial N_{2}^{(i)}}{\partial f_{h t}} \equiv a_{2}^{i, 10}=-\left(T_{w}^{(i+1)}-T_{a}^{(i)}\right) \frac{\partial H\left(m_{a}, \alpha\right)}{\partial f_{h t}} ; \quad \ell=2 ; i=1, \ldots, I ; j=10,
$$

where:

$$
\frac{\partial H\left(m_{a}, \boldsymbol{\alpha}\right)}{\partial f_{h t}}=\frac{H\left(m_{a}, \boldsymbol{\alpha}\right)}{f_{h t}}=\frac{k_{\text {air }} N u(\operatorname{Re}, \boldsymbol{\alpha}) w_{t s a} A_{\text {surf }}}{\mathrm{D}_{h} I} .
$$

The derivatives of the liquid energy balance equations [cf. Equations (A4)-(A6)] with respect to the parameter $\alpha^{(11)} \equiv f_{m t}$ are as follows:

$$
\frac{\partial N_{2}^{(i)}}{\partial \alpha^{(11)}}=\frac{\partial N_{2}^{(i)}}{\partial f_{m t}} \equiv a_{2}^{i, 11}=0 ; \quad \ell=2 ; i=1, \ldots, I ; j=11 .
$$

The derivatives of the liquid energy balance equations [cf. Equations (A4)-(A6)] with respect to the parameter $\alpha^{(12)} \equiv f$ are as follows:

$$
\frac{\partial N_{2}^{(i)}}{\partial \alpha^{(12)}}=\frac{\partial N_{2}^{(i)}}{\partial f} \equiv a_{2}^{i, 12}=0 ; \quad \ell=2 ; i=1, \ldots, I ; j=12 .
$$

The derivatives of the liquid energy balance equations [cf. Equations (A4)-(A6)] with respect to the parameter $\alpha^{(13)} \equiv a_{0}$ are as follows:

$$
\frac{\partial N_{2}^{(i)}}{\partial \alpha^{(13)}}=\frac{\partial N_{2}^{(i)}}{\partial a_{0}} \equiv a_{2}^{i, 13}=0 ; \quad \ell=2 ; i=1, \ldots, I ; j=13 .
$$

The derivatives of the liquid energy balance equations [cf. Equations (A4)-(A6)] with respect to the parameter $\alpha^{(14)} \equiv a_{1}$ are as follows:

$$
\frac{\partial N_{2}^{(i)}}{\partial \alpha^{(14)}}=\frac{\partial N_{2}^{(i)}}{\partial a_{1}} \equiv a_{2}^{i, 14}=0 ; \quad \ell=2 ; i=1, \ldots, I ; j=14 .
$$

The derivatives of the liquid energy balance equations [cf. Equations (A4)-(A6)] with respect to the parameter $\alpha^{(15)} \equiv a_{0, \text { cpa }}$ are as follows:

$$
\frac{\partial N_{2}^{(i)}}{\partial \alpha^{(15)}}=\frac{\partial N_{2}^{(i)}}{\partial a_{0, c p a}} \equiv a_{2}^{i, 15}=0 ; \quad \ell=2 ; i=1, \ldots, I ; j=15 .
$$

The derivatives of the liquid energy balance equations [cf. Equations (A4)-(A6)] with respect to the parameter $\alpha^{(16)} \equiv a_{1, \text { cpa }}$ are as follows:

$$
\frac{\partial N_{2}^{(i)}}{\partial \alpha^{(16)}}=\frac{\partial N_{2}^{(i)}}{\partial a_{1, c p a}} \equiv a_{2}^{i, 16}=0 ; \quad \ell=2 ; i=1, \ldots, I ; j=16 .
$$

The derivatives of the liquid energy balance equations [cf. Equations (A4)-(A6)] with respect to the parameter $\alpha^{(17)} \equiv a_{2, \text { cpa }}$ are as follows:

$$
\frac{\partial N_{2}^{(i)}}{\partial \alpha^{(17)}}=\frac{\partial N_{2}^{(i)}}{\partial a_{2, c p a}} \equiv a_{2}^{i, 17}=0 ; \quad \ell=2 ; i=1, \ldots, I ; j=17 .
$$


The derivatives of the liquid energy balance equations [cf. Equations (A4)-(A6)] with respect to the parameter $\alpha^{(18)} \equiv a_{0, \text { dav }}$ are as follows:

$$
\frac{\partial N_{2}^{(i)}}{\partial \alpha^{(18)}}=\frac{\partial N_{2}^{(i)}}{\partial a_{0, d a v}} \equiv a_{2}^{i, 18}=0 ; \quad \ell=2 ; i=1, \ldots, I ; j=18 .
$$

The derivatives of the liquid energy balance equations [cf. Equations (A4)-(A6)] with respect to the parameter $\alpha^{(19)} \equiv a_{1, \text { dav }}$ are as follows:

$$
\frac{\partial N_{2}^{(i)}}{\partial \alpha^{(19)}}=\frac{\partial N_{2}^{(i)}}{\partial a_{1, \text { dav }}} \equiv a_{2}^{i, 19}=0 ; \ell=2 ; i=1, \ldots, I ; j=19 .
$$

The derivatives of the liquid energy balance equations [cf. Equations (A4)-(A6)] with respect to the parameter $\alpha^{(20)} \equiv a_{2, \text { dav }}$ are as follows:

$$
\frac{\partial N_{2}^{(i)}}{\partial \alpha^{(20)}}=\frac{\partial N_{2}^{(i)}}{\partial a_{2, \text { dav }}} \equiv a_{2}^{i, 20}=0 ; \quad \ell=2 ; i=1, \ldots, I ; j=20 .
$$

The derivatives of the liquid energy balance equations [cf. Equations (A4)-(A6)] with respect to the parameter $\alpha^{(21)} \equiv a_{3, \text { dav }}$ are as follows:

$$
\frac{\partial N_{2}^{(i)}}{\partial \alpha^{(21)}}=\frac{\partial N_{2}^{(i)}}{\partial a_{3, d a v}} \equiv a_{2}^{i, 21}=0 ; \quad \ell=2 ; i=1, \ldots, I ; j=21 .
$$

The derivatives of the liquid energy balance equations [cf. Equations (A4)-(A6)] with respect to the parameter $\alpha^{(22)} \equiv a_{0 f}$ are as follows:

$$
\begin{gathered}
\frac{\partial N_{2}^{(i)}}{\partial \alpha^{(22)}}=\frac{\partial N_{2}^{(i)}}{\partial a_{0 f}} \equiv a_{2}^{i, 22}=m_{w}^{(i)} \frac{\partial h_{f}^{(i)}\left(T_{w}^{(i)}, \alpha\right)}{\partial a_{0 f}}-m_{w}^{(i+1)} \frac{\partial h_{f}^{(i+1)}\left(T_{w}^{(i+1)}, \alpha\right)}{\partial a_{0 f}}=m_{w}^{(i)}-m_{w}^{(i+1)} ; \\
\ell=2 ; i=1, \ldots, I ; j=22 .
\end{gathered}
$$

The derivatives of the liquid energy balance equations [cf. Equations (A4)-(A6)] with respect to the parameter $\alpha^{(23)} \equiv a_{1 f}$ are as follows:

$$
\begin{aligned}
& \frac{\partial N_{2}^{(i)}}{\partial \alpha^{(23)}}=\frac{\partial N_{2}^{(i)}}{\partial a_{1 f}} \equiv a_{2}^{i, 23}=m_{w}^{(i)} \frac{\partial h_{f}^{(i)}\left(T_{w}^{(i)}, \alpha\right)}{\partial a_{1 f}}-m_{w}^{(i+1)} \frac{\partial h_{f}^{(i+1)}\left(T_{w}^{(i+1)}, \alpha\right)}{\partial a_{1 f}} \\
& \quad=T_{w}^{(i)} m_{w}^{(i)}-T_{w}^{(i+1)} m_{w}^{(i+1)} ; \quad \ell=2 ; i=1, \ldots, I ; j=23 .
\end{aligned}
$$

The derivatives of the liquid energy balance equations [cf. Equations (A4)-(A6)] with respect to the parameter $\alpha^{(24)} \equiv a_{0 g}$ are as follows:

$$
\begin{gathered}
\frac{\partial N_{2}^{(i)}}{\partial \alpha^{(24)}}=\frac{\partial N_{2}^{(i)}}{\partial a_{0 g}} \equiv a_{2}^{i, 24}=-\left(m_{w}^{(i)}-m_{w}^{(i+1)}\right) \frac{\partial h_{g, w}^{(i+1)}\left(T_{w}^{(i+1)}, \alpha\right)}{\partial a_{0 g}}=m_{w}^{(i+1)}-m_{w}^{(i)} \\
\ell=2 ; i=1, \ldots, I ; j=24 .
\end{gathered}
$$

The derivatives of the liquid energy balance equations [cf. Equations (A4)-(A6)] with respect to the parameter $\alpha^{(25)} \equiv a_{1 g}$ are as follows:

$$
\begin{gathered}
\frac{\partial N_{2}^{(i)}}{\partial \alpha^{(25)}}=\frac{\partial N_{2}^{(i)}}{\partial a_{1 g}} \equiv a_{2}^{i, 25}=-\left(m_{w}^{(i)}-m_{w}^{(i+1)}\right) \frac{\partial h_{g, w}^{(i+1)}\left(T_{w}^{(i+1)}, \alpha\right)}{\partial a_{1 g}}=-\left(m_{w}^{(i)}-m_{w}^{(i+1)}\right) T_{w}^{(i+1)} ; \\
\ell=2 ; i=1, \ldots, I ; j=25 .
\end{gathered}
$$


The derivatives of the liquid energy balance equations [cf. Equations (A4)-(A6)] with respect to the parameter $\alpha^{(26)} \equiv a_{0, N u}$ are as follows:

$$
\frac{\partial N_{2}^{(i)}}{\partial \alpha^{(26)}}=\frac{\partial N_{2}^{(i)}}{\partial a_{0, N u}} \equiv a_{2}^{i, 26}=-\left(T_{w}^{(i+1)}-T_{a}^{(i)}\right) \frac{\partial H\left(m_{a}, \alpha\right)}{\partial N u(\operatorname{Re}, \alpha)} \frac{\partial N u(\operatorname{Re}, \alpha)}{\partial a_{0, N u}} ; \quad \ell=2 ; i=1, \ldots, I ; j=26,
$$

where $\frac{\partial H\left(m_{a}, \boldsymbol{\alpha}\right)}{\partial N u(\operatorname{Re}, \boldsymbol{\alpha})}$ was defined in Equation (A105) and $\frac{\partial N u(\operatorname{Re}, \boldsymbol{\alpha})}{\partial a_{0, N} u}$ was defined in Equation (A59).

The derivatives of the liquid energy balance equations [cf. Equations (A4)-(A6)] with respect to the parameter $\alpha^{(27)} \equiv a_{1, \mathrm{~N} u}$ are as follows:

$$
\begin{gathered}
\frac{\partial N_{2}^{(i)}}{\partial \alpha^{(27)}}=\frac{\partial N_{2}^{(i)}}{\partial a_{1, N u}} \equiv a_{2}^{i, 27}=-\left(T_{w}^{(i+1)}-T_{a}^{(i)}\right) \frac{\partial H\left(m_{a}, \alpha\right)}{\partial N u(\operatorname{Re}, \alpha)} \frac{\partial N u(\operatorname{Re}, \alpha)}{\partial a_{1, N u}} ; \\
\quad \ell=2 ; i=1, \ldots, I ; j=27,
\end{gathered}
$$

where $\frac{\partial H\left(m_{a}, \boldsymbol{\alpha}\right)}{\partial N u(\operatorname{Re}, \boldsymbol{\alpha})}$ was defined in Equation (A105) and $\frac{\partial N u(\mathrm{Re}, \boldsymbol{\alpha})}{\partial a_{1, N u}}$ was defined in Equation (A61).

The derivatives of the liquid energy balance equations [cf. Equations (A4)-(A6)] with respect to the parameter $\alpha^{(28)} \equiv a_{2, N u}$ are as follows:

$$
\begin{aligned}
& \frac{\partial N_{2}^{(i)}}{\partial \alpha^{(28)}}=\frac{\partial N_{2}^{(i)}}{\partial a_{2, N u}} \equiv a_{2}^{i, 28}=-\left(T_{w}^{(i+1)}-T_{a}^{(i)}\right) \frac{\partial H\left(m_{a}, \alpha\right)}{\partial N u(\operatorname{Re}, \alpha)} \frac{\partial N u(\operatorname{Re}, \alpha)}{\partial a_{2, N u}} ; \\
& \ell=2 ; i=1, \ldots, I ; j=28 \text {, }
\end{aligned}
$$

where $\frac{\partial H\left(m_{a}, \boldsymbol{\alpha}\right)}{\partial N u(\operatorname{Re}, \boldsymbol{\alpha})}$ was defined in Equation (A105) and $\frac{\partial N u(\mathrm{Re}, \boldsymbol{\alpha})}{\partial a_{2, N u}}$ was defined in Equation (A63).

The derivatives of the liquid energy balance equations [cf. Equations (A4)-(A6)] with respect to the parameter $\alpha^{(29)} \equiv a_{3, N u}$ are as follows:

$$
\begin{gathered}
\frac{\partial N_{2}^{(i)}}{\partial \alpha^{(29)}}=\frac{\partial N_{2}^{(i)}}{\partial a_{3, N u}} \equiv a_{2}^{i, 29}=-\left(T_{w}^{(i+1)}-T_{a}^{(i)}\right) \frac{\partial H\left(m_{a}, \alpha\right)}{\partial N u(\operatorname{Re}, \alpha)} \frac{\partial N u(\operatorname{Re}, \alpha)}{\partial a_{3, N u}} ; \\
\ell=2 ; i=1, \ldots, I ; j=29,
\end{gathered}
$$

where $\frac{\partial H\left(m_{a}, \boldsymbol{\alpha}\right)}{\partial N u(\operatorname{Re}, \boldsymbol{\alpha})}$ was defined in Equation (A105) and $\frac{\partial N u(\operatorname{Re}, \boldsymbol{\alpha})}{\partial a_{3, N u}}$ was defined in Equation (A65).

The derivatives of the liquid energy balance equations [cf. Equations (A4)-(A6)] with respect to the parameter $\alpha^{(30)} \equiv W_{d k x}$ are as follows:

$$
\frac{\partial N_{2}^{(i)}}{\partial \alpha^{(30)}}=\frac{\partial N_{2}^{(i)}}{\partial W_{d k x}} \equiv a_{2}^{i, 30}=0 ; \quad \ell=2 ; i=1, \ldots, I ; j=30 .
$$

The derivatives of the liquid energy balance equations [cf. Equations (A4)-(A6)] with respect to the parameter $\alpha^{(31)} \equiv W_{d k y}$ are as follows:

$$
\frac{\partial N_{2}^{(i)}}{\partial \alpha^{(31)}}=\frac{\partial N_{2}^{(i)}}{\partial W_{d k y}} \equiv a_{2}^{i, 31}=0 ; \quad \ell=2 ; i=1, \ldots, I ; j=31 .
$$

The derivatives of the liquid energy balance equations [cf. Equations (A4)-(A6)] with respect to the parameter $\alpha^{(32)} \equiv \Delta z_{d k}$ are as follows:

$$
\frac{\partial N_{2}^{(i)}}{\partial \alpha^{(32)}}=\frac{\partial N_{2}^{(i)}}{\partial \Delta z_{d k}} \equiv a_{2}^{i, 32}=0 ; \quad \ell=2 ; i=1, \ldots, I ; j=32 .
$$

The derivatives of the liquid energy balance equations [cf. Equations (A4)-(A6)] with respect to the parameter $\alpha^{(33)} \equiv \Delta z_{f a n}$ are as follows:

$$
\frac{\partial N_{2}^{(i)}}{\partial \alpha^{(33)}}=\frac{\partial N_{2}^{(i)}}{\partial \Delta z_{f a n}} \equiv a_{2}^{i, 33}=0 ; \quad \ell=2 ; i=1, \ldots, I ; j=33 .
$$


The derivatives of the liquid energy balance equations [cf. Equations (A4)-(A6)] with respect to the parameter $\alpha^{(34)} \equiv D_{\text {fan }}$ are as follows:

$$
\begin{aligned}
\frac{\partial N_{2}^{(i)}}{\partial \alpha^{(34)}}=\frac{\partial N_{2}^{(i)}}{\partial D_{f a n}} \equiv a_{2}^{i, 34} & =-\left(T_{w}^{(i+1)}-T_{a}^{(i)}\right) \frac{\partial H\left(m_{a}, \alpha\right)}{\partial N u(\operatorname{Re}, \alpha)} \frac{\partial N u(\operatorname{Re}, \alpha)}{\partial m_{a}} \frac{\partial m_{a}}{\partial D_{f a n}} \\
\ell & =2 ; i=1, \ldots, I ; j=34
\end{aligned}
$$

where $\frac{\partial H\left(m_{a}, \boldsymbol{\alpha}\right)}{\partial N u(\operatorname{Re}, \boldsymbol{\alpha})}$ was defined in Equation (A105), while $\frac{\partial N u(\operatorname{Re}, \boldsymbol{\alpha})}{\partial m_{a}}$ and $\frac{\partial m_{a}}{\partial D_{f a n}}$ were defined in Equations (A24) and (A71), respectively.

The derivatives of the liquid energy balance equations [cf. Equations (A4)-(A6)] with respect to the parameter $\alpha^{(35)} \equiv \Delta z_{\text {fill }}$ are as follows:

$$
\frac{\partial N_{2}^{(i)}}{\partial \alpha^{(35)}}=\frac{\partial N_{2}^{(i)}}{\partial \Delta z_{\text {fill }}} \equiv a_{2}^{i, 35}=0 ; \quad \ell=2 ; i=1, \ldots, I ; j=35 .
$$

The derivatives of the liquid energy balance equations [cf. Equations (A4)-(A6)] with respect to the parameter $\alpha^{(36)} \equiv \Delta z_{\text {rain }}$ are as follows:

$$
\frac{\partial N_{2}^{(i)}}{\partial \alpha^{(36)}}=\frac{\partial N_{2}^{(i)}}{\partial \Delta z_{\text {rain }}} \equiv a_{2}^{i, 36}=0 ; \quad \ell=2 ; i=1, \ldots, I ; j=36
$$

The derivatives of the liquid energy balance equations [cf. Equations (A4)-(A6)] with respect to the parameter $\alpha^{(37)} \equiv \Delta z_{b s}$ are as follows:

$$
\frac{\partial N_{2}^{(i)}}{\partial \alpha^{(37)}}=\frac{\partial N_{2}^{(i)}}{\partial \Delta z_{b s}} \equiv a_{2}^{i, 37}=0 ; \quad \ell=2 ; i=1, \ldots, I ; j=37 .
$$

The derivatives of the liquid energy balance equations [cf. Equations (A4)-(A6)] with respect to the parameter $\alpha^{(38)} \equiv \Delta z_{d e}$ are as follows:

$$
\frac{\partial N_{2}^{(i)}}{\partial \alpha^{(38)}}=\frac{\partial N_{2}^{(i)}}{\partial \Delta z_{d e}} \equiv a_{2}^{i, 38}=0 ; \quad \ell=2 ; i=1, \ldots, I ; j=38 .
$$

The derivatives of the liquid energy balance equations [cf. Equations (A4)-(A6)] with respect to the parameter $\alpha^{(39)} \equiv D_{h}$ are as follows:

$$
\frac{\partial N_{2}^{(i)}}{\partial \alpha^{(39)}}=\frac{\partial N_{2}^{(i)}}{\partial D_{h}} \equiv a_{2}^{i, 39}=-\left(T_{w}^{(i+1)}-T_{a}^{(i)}\right) \frac{\partial H\left(m_{a}, \alpha\right)}{\partial D_{h}} ; \quad \ell=2 ; i=1, \ldots, I ; j=39,
$$

where

$$
\frac{\partial H\left(m_{a}, \boldsymbol{\alpha}\right)}{\partial D_{h}}= \begin{cases}-H\left(m_{a}, \boldsymbol{\alpha}\right) / D_{h} & \operatorname{Re}_{d}<2300 \\ -\frac{a_{2, N u} H\left(m_{a}, \boldsymbol{\alpha}\right)}{N u(\operatorname{Re}, \boldsymbol{\alpha}) D_{h}} & 2300 \leq \operatorname{Re}_{d} \leq 10000 \\ -0.2 \cdot H\left(m_{a}, \boldsymbol{\alpha}\right) / D_{h} & \operatorname{Re}_{d}>10000\end{cases}
$$

The derivatives of the liquid energy balance equations [cf. Equations (A4)-(A6)] with respect to the parameter $\alpha^{(40)} \equiv A_{\text {fill }}$ are as follows:

$$
\frac{\partial N_{2}^{(i)}}{\partial \alpha^{(40)}}=\frac{\partial N_{2}^{(i)}}{\partial A_{\text {fill }}} \equiv a_{2}^{i, 40}=-\left(T_{w}^{(i+1)}-T_{a}^{(i)}\right) \frac{\partial H\left(m_{a}, \alpha\right)}{\partial A_{\text {fill }}} ; \quad \ell=2 ; i=1, \ldots, I ; j=40,
$$

where:

$$
\frac{\partial H\left(m_{a}, \boldsymbol{\alpha}\right)}{\partial A_{\text {fill }}}= \begin{cases}0 & \operatorname{Re}_{d}<2300 \\ -\frac{a_{1, N u} H\left(m_{a}, \boldsymbol{\alpha}\right) \operatorname{Re}\left(m_{a}, \boldsymbol{\alpha}\right)}{N u(\operatorname{Re}, \boldsymbol{\alpha}) A_{\text {fill }}} & 2300 \leq \operatorname{Re}_{d} \leq 10000 \\ -0.8 \cdot H\left(m_{a}, \boldsymbol{\alpha}\right) / A_{\text {fill }} & \operatorname{Re}_{d}>10000\end{cases}
$$


The derivatives of the liquid energy balance equations [cf. Equations (A4)-(A6)] with respect to the parameter $\alpha^{(41)} \equiv A_{\text {surf }}$ are as follows:

$$
\frac{\partial N_{2}^{(i)}}{\partial \alpha^{(41)}}=\frac{\partial N_{2}^{(i)}}{\partial A_{\text {surf }}} \equiv a_{2}^{i, 41}=-\left(T_{w}^{(i+1)}-T_{a}^{(i)}\right) \frac{\partial H\left(m_{a}, \alpha\right)}{\partial A_{\text {surf }}} ; \quad \ell=2 ; i=1, \ldots, I ; j=41,
$$

where:

$$
\frac{\partial H\left(m_{a}, \boldsymbol{\alpha}\right)}{\partial A_{\text {surf }}}=\frac{H\left(m_{a}, \boldsymbol{\alpha}\right)}{A_{\text {surf }}}=\frac{f_{h t} k_{\text {air }} N u(\operatorname{Re}, \boldsymbol{\alpha}) w_{t s a}}{\mathrm{D}_{h} I} .
$$

The derivatives of the liquid energy balance equations [cf. Equations (A4)-(A6)] with respect to the parameter $\alpha^{(42)} \equiv \operatorname{Pr}$ are as follows:

$$
\frac{\partial N_{2}^{(i)}}{\partial \alpha^{(42)}}=\frac{\partial N_{2}^{(i)}}{\partial \operatorname{Pr}} \equiv a_{2}^{i, 42}=-\left(T_{w}^{(i+1)}-T_{a}^{(i)}\right) \frac{\partial H\left(m_{a}, \alpha\right)}{\partial \operatorname{Pr}} ; \quad \ell=2 ; i=1, \ldots, I ; j=42,
$$

where:

$$
\frac{\partial H\left(m_{a}, \boldsymbol{\alpha}\right)}{\partial \operatorname{Pr}}= \begin{cases}0 & \operatorname{Re}_{d} \leq 10000 \\ H\left(m_{a}, \boldsymbol{\alpha}\right) /(3 \cdot \operatorname{Pr}) & \operatorname{Re}_{d}>10000\end{cases}
$$

The derivatives of the liquid energy balance equations [cf. Equations (A4)-(A6)] with respect to the parameter $\alpha^{(33)} \equiv V_{w}$ are as follows:

$$
\frac{\partial N_{2}^{(i)}}{\partial \alpha^{(43)}}=\frac{\partial N_{2}^{(i)}}{\partial V_{w}} \equiv a_{2}^{i, 43}=0 ; \quad \ell=2 ; i=1, \ldots, I ; j=43 .
$$

The derivatives of the liquid energy balance equations [cf. Equations (A4)-(A6)] with respect to the parameter $\alpha^{(44)} \equiv V_{\text {exit }}$ are as follows:

$$
\begin{aligned}
\frac{\partial N_{2}^{(i)}}{\partial \alpha^{(44)}}=\frac{\partial N_{2}^{(i)}}{\partial V_{\text {exit }}} \equiv a_{2}^{i, 44} & =-\left(T_{w}^{(i+1)}-T_{a}^{(i)}\right) \frac{\partial H\left(m_{a}, \alpha\right)}{\partial N u(\operatorname{Re}, \alpha)} \frac{\partial N u(\operatorname{Re}, \alpha)}{\partial m_{a}} \frac{\partial m_{a}}{\partial V_{\text {exit }}} \\
\ell & =2 ; i=1, \ldots, I ; j=44
\end{aligned}
$$

where $\frac{\partial H\left(m_{a}, \boldsymbol{\alpha}\right)}{\partial N u(\operatorname{Re}, \boldsymbol{\alpha})}$ was defined in Equation (A105), while $\frac{\partial N u(\mathrm{Re}, \boldsymbol{\alpha})}{\partial m_{a}}$ and $\frac{\partial m_{a}}{\partial V_{e x i t}}$ and were defined previously in Equations (A24) and (A86), respectively.

The derivatives of the liquid energy balance equations [cf. Equations (A4)-(A6)] with respect to the parameter $\alpha^{(45)} \equiv m_{w, i n}$ are as follows:

$$
\begin{gathered}
\frac{\partial N_{2}^{(1)}}{\partial \alpha^{(45)}}=\frac{\partial N_{2}^{(1)}}{\partial m_{w, i n}} \equiv a_{2}^{1,45}=h_{f}^{(1)}\left(T_{w, i n}, \alpha\right)-h_{g, w}^{(2)}\left(T_{w}^{(2)}, \alpha\right)=T_{w, i n} a_{1 f}-a_{1 g} T_{w}^{(2)}+a_{0 f}-a_{0 g}, \\
\ell=2 ; i=1 ; j=45, \\
\frac{\partial N_{2}^{(i)}}{\partial \alpha^{(45)}}=\frac{\partial N_{2}^{(i)}}{\partial m_{w, i n}} \equiv a_{2}^{i, 45}=0 ; \quad \ell=2 ; i=2, \ldots, I ; j=45 .
\end{gathered}
$$

The derivatives of the liquid energy balance equations [cf. Equations (A4)-(A6)] with respect to the parameter $\alpha^{(46)} \equiv T_{a, i n}$ are as follows:

$$
\frac{\partial N_{2}^{(i)}}{\partial \alpha^{(46)}}=\frac{\partial N_{2}^{(i)}}{\partial T_{a, i n}} \equiv a_{2}^{i, 46}=0 ; \quad \ell=2 ; i=1, \ldots, I ; j=46 .
$$


The derivatives of the liquid energy balance equations [cf. Equations (A4)-(A6)] with respect to the parameter $\alpha^{(47)} \equiv m_{a}$ are as follows:

$$
\begin{gathered}
\frac{\partial N_{2}^{(i)}}{\partial \alpha^{(47)}}=\frac{\partial N_{2}^{(i)}}{\partial m_{a}} \equiv a_{2}^{i, 47}=-\left(T_{w}^{(i+1)}-T_{a}^{(i)}\right) \frac{\partial H\left(m_{a}, \alpha\right)}{\partial N u(\operatorname{Re}, \alpha)} \frac{\partial N u(\operatorname{Re}, \alpha)}{\partial m_{a}} \\
\ell=2 ; i=1, \ldots, I ; j=47
\end{gathered}
$$

where $\frac{\partial H\left(m_{a}, \boldsymbol{\alpha}\right)}{\partial N u(\operatorname{Re}, \boldsymbol{\alpha})}$ and $\frac{\partial N u(\operatorname{Re}, \boldsymbol{\alpha})}{\partial m_{a}}$ were defined previously in Equations (A24) and (A105), respectively.

The derivatives of the liquid energy balance equations [cf. Equations (A4)-(A6)] with respect to the parameter $\alpha^{(48)} \equiv \omega_{\text {in }}$ are as follows:

$$
\frac{\partial N_{2}^{(i)}}{\partial \alpha^{(48)}}=\frac{\partial N_{2}^{(i)}}{\partial \omega_{i n}} \equiv a_{2}^{i, 48}=0 ; \quad \ell=2 ; i=1, \ldots, I ; j=48 .
$$

The derivatives of the liquid energy balance equations [cf. Equations (A4)-(A6)] with respect to the parameter $\alpha^{(49)} \equiv \operatorname{Re}_{d}$ are as follows:

$$
\begin{gathered}
\frac{\partial N_{2}^{(i)}}{\partial \alpha^{(49)}}=\frac{\partial N_{2}^{(i)}}{\partial \operatorname{Re}_{d}} \equiv a_{2}^{i, 49}=-\left(T_{w}^{(i+1)}-T_{a}^{(i)}\right) \frac{\partial H\left(m_{a}, \alpha\right)}{\partial N u\left(\operatorname{Re}_{d}, \alpha\right)} \frac{\partial N u\left(\operatorname{Re}_{d}, \alpha\right)}{\partial \operatorname{Re}_{d}} \\
\ell=2 ; i=1, \ldots, I ; j=49
\end{gathered}
$$

where $\frac{\partial H\left(m_{a}, \boldsymbol{\alpha}\right)}{\partial N u(\operatorname{Re} \boldsymbol{\alpha})}$ was defined in Equation (A105), and $\frac{\partial N u(\mathrm{Re}, \boldsymbol{\alpha})}{\partial m_{a}}$ was defined in Equation (A93).

The derivatives of the liquid energy balance equations [cf. Equations (A4)-(A6)] with respect to the parameter $\alpha^{(50)} \equiv S c$ are as follows:

$$
\frac{\partial N_{2}^{(i)}}{\partial \alpha^{(50)}}=\frac{\partial N_{2}^{(i)}}{\partial S c} \equiv a_{2}^{i, 50}=0 ; \quad \ell=2 ; i=1, \ldots, I ; j=50 .
$$

The derivatives of the liquid energy balance equations [cf. Equations (A4)-(A6)] with respect to the parameter $\alpha^{(51)} \equiv S h$ are as follows:

$$
\frac{\partial N_{2}^{(i)}}{\partial \alpha^{(51)}}=\frac{\partial N_{2}^{(i)}}{\partial S h} \equiv a_{2}^{i, 51}=0 ; \quad \ell=2 ; i=1, \ldots, I ; j=51 .
$$

The derivatives of the liquid energy balance equations [cf. Equations (A4)-(A6)] with respect to the parameter $\alpha^{(52)} \equiv N u$ are as follows:

$$
\frac{\partial N_{2}^{(i)}}{\partial \alpha^{(52)}}=\frac{\partial N_{2}^{(i)}}{\partial N u} \equiv a_{2}^{i, 52}=-\left(T_{w}^{(i+1)}-T_{a}^{(i)}\right) \frac{\partial H\left(m_{a}, \alpha\right)}{\partial N u} ; \quad \ell=2 ; i=1, \ldots, I ; j=52,
$$

where $\frac{\partial H\left(m_{a}, \boldsymbol{\alpha}\right)}{\partial N u(\operatorname{Re}, \boldsymbol{\alpha})}$ was defined in Equation (A105).

\section{A3. Derivatives of the Water Vapor Continuity Equations with Respect to the Parameters}

The derivatives of the water vapor continuity equations [cf. Equations (A7)-(A9)] with respect to the parameter $\alpha^{(1)} \equiv T_{d b}$ are as follows:

$$
\frac{\partial N_{3}^{(i)}}{\partial \alpha^{(1)}}=\frac{\partial N_{3}^{(i)}}{\partial T_{d b}} \equiv a_{3}^{i, 1}=0 ; \quad \ell=3 ; i=1, \ldots, I ; j=1 .
$$

The derivatives of the water vapor continuity equations [cf. Equations (A7)-(A9)] with respect to the parameter $\alpha^{(2)} \equiv T_{d p}$ are as follows:

$$
\frac{\partial N_{3}^{(i)}}{\partial \alpha^{(2)}}=\frac{\partial N_{3}^{(i)}}{\partial T_{d p}} \equiv a_{3}^{i, 2}=0 ; \quad \ell=3 ; i=1, \ldots, I-1 ; j=2,
$$




$$
\frac{\partial N_{3}^{(I)}}{\partial \alpha^{(2)}}=\frac{\partial N_{3}^{(I)}}{\partial T_{d p}} \equiv a_{3}^{I, 2}=\frac{\partial \omega_{i n}}{\partial T_{d p}} ; \quad \ell=3 ; i=I ; j=2,
$$

where:

$$
\frac{\partial \omega_{i n}}{\partial T_{d p}}=-\frac{0.622 a_{1} P_{a t m} \mathrm{e}^{a_{0}+\frac{a_{1}}{T_{d p}}}}{T_{t d p}{ }^{2}\left(P_{a t m}-\mathrm{e}^{a_{0}+\frac{a_{1}}{T_{d p}}}\right)^{2}} .
$$

The derivatives of the water vapor continuity equations [cf. Equations (A7)-(A9)] with respect to the parameter $\alpha^{(3)} \equiv T_{w, i n}$ are as follows:

$$
\frac{\partial N_{3}^{(1)}}{\partial \alpha^{(3)}}=\frac{\partial N_{3}^{(1)}}{\partial T_{w, i n}} \equiv a_{3}^{1,3}=\frac{1}{m_{a}} \frac{\partial m_{w, i n}}{\partial T_{w, i n}} ; \quad \ell=3 ; i=1 ; j=3,
$$

where $\frac{\partial w, \text { in }}{\partial T_{w, i n}}$ was defined in Equation (A20).

$$
\frac{\partial N_{3}^{(i)}}{\partial \alpha^{(3)}}=\frac{\partial N_{3}^{(i)}}{\partial T_{w, i n}} \equiv a_{3}^{i, 3}=0 ; \quad \ell=3 ; i=2, \ldots I ; j=3 .
$$

The derivatives of the water vapor continuity equations [cf. Equations (A7)-(A9)] with respect to the parameter $\alpha^{(4)} \equiv P_{a t m}$ are as follows:

$$
\begin{gathered}
\frac{\partial N_{3}^{(i)}}{\partial \alpha^{(4)}}=\frac{\partial N_{3}^{(i)}}{\partial P_{a t m}} \equiv a_{3}^{i, 4}=-\frac{m_{w}^{(i)}-m_{w}^{(i+1)}}{m_{a}{ }^{2}} \frac{\partial m_{a}}{\partial P_{a t m}} ; \quad \ell=3 ; i=1, \ldots, I-1 ; j=4, \\
\frac{\partial N_{3}^{(I)}}{\partial \alpha^{(4)}}=\frac{\partial N_{3}^{(I)}}{\partial P_{a t m}} \equiv a_{3}^{I, 4}=\frac{\partial \omega_{i n}}{\partial P_{a t m}}-\frac{m_{w}^{(i)}-m_{w}^{(i+1)}}{m_{a}{ }^{2}} \frac{\partial m_{a}}{\partial P_{a t m}} ; \quad \ell=3 ; i=I ; j=4,
\end{gathered}
$$

where $\frac{\partial m_{a}}{\partial P_{a t m}}$ was defined in Equation (A25) and:

$$
\frac{\partial \omega_{i n}}{\partial P_{a t m}}=-\frac{0.622 \mathrm{e}^{a_{0}+\frac{a_{1}}{T_{d p}}}}{\left(P_{a t m}-\mathrm{e}^{a_{0}+\frac{a_{1}}{T_{d p}}}\right)^{2}} .
$$

The derivatives of the water vapor continuity equations [cf. Equations (A7)-(A9)] with respect to the parameter $\alpha^{(5)} \equiv w_{t s a}$ are as follows:

$$
\frac{\partial N_{3}^{(i)}}{\partial \alpha^{(5)}}=\frac{\partial N_{3}^{(i)}}{\partial w_{t s a}} \equiv a_{3}^{i, 5}=0 ; \quad \ell=3 ; i=1, \ldots, I ; j=5 .
$$

The derivatives of the water vapor continuity equations [cf. Equations (A7)-(A9)] with respect to the parameter $\alpha^{(6)} \equiv k_{\text {sum }}$ are as follows:

$$
\frac{\partial N_{3}^{(i)}}{\partial \alpha^{(6)}}=\frac{\partial N_{3}^{(i)}}{\partial k_{\text {sum }}} \equiv a_{3}^{i, 6}=0 ; \quad \ell=3 ; i=1, \ldots, I ; j=6 .
$$

The derivatives of the water vapor continuity equations [cf. Equations (A7)-(A9)] with respect to the parameter $\alpha^{(7)} \equiv \mu$ are as follows:

$$
\frac{\partial N_{3}^{(i)}}{\partial \alpha(7)}=\frac{\partial N_{3}^{(i)}}{\partial \mu} \equiv a_{3}^{i, 7}=0 ; \quad \ell=3 ; i=1, \ldots, I ; j=7 .
$$


The derivatives of the water vapor continuity equations [cf. Equations (A7)-(A9)] with respect to the parameter $\alpha^{(8)} \equiv v$ are as follows:

$$
\frac{\partial N_{3}^{(i)}}{\partial \alpha^{(8)}}=\frac{\partial N_{3}^{(i)}}{\partial v} \equiv a_{3}^{i, 8}=0 ; \quad \ell=3 ; i=1, \ldots, I ; j=8 .
$$

The derivatives of the water vapor continuity equations [cf. Equations (A7)-(A9)] with respect to the parameter $\alpha^{(9)} \equiv k_{\text {air }}$ are as follows:

$$
\frac{\partial N_{3}^{(i)}}{\partial \alpha^{(9)}}=\frac{\partial N_{3}^{(i)}}{\partial k_{\text {air }}} \equiv a_{3}^{i, 9}=0 ; \quad \ell=3 ; i=1, \ldots, I ; j=9 .
$$

The derivatives of the water vapor continuity equations [cf. Equations (A7)-(A9)] with respect to the parameter $\alpha^{(10)} \equiv f_{h t}$ are as follows:

$$
\frac{\partial N_{3}^{(i)}}{\partial \alpha^{(10)}}=\frac{\partial N_{3}^{(i)}}{\partial f_{h t}} \equiv a_{3}^{i, 10}=0 ; \quad \ell=3 ; i=1, \ldots, I ; j=10 .
$$

The derivatives of the water vapor continuity equations [cf. Equations (A7)-(A9)] with respect to the parameter $\alpha^{(11)} \equiv f_{m t}$ are as follows:

$$
\frac{\partial N_{3}^{(i)}}{\partial \alpha^{(11)}}=\frac{\partial N_{3}^{(i)}}{\partial f_{m t}} \equiv a_{3}^{i, 11}=0 ; \ell=3 ; i=1, \ldots, I ; j=11 .
$$

The derivatives of the water vapor continuity equations [cf. Equations (A7)-(A9)] with respect to the parameter $\alpha^{(12)} \equiv f$ are as follows:

$$
\frac{\partial N_{3}^{(i)}}{\partial \alpha^{(12)}}=\frac{\partial N_{3}^{(i)}}{\partial f} \equiv a_{3}^{i, 12}=0 ; \quad \ell=3 ; i=1, \ldots, I ; j=12 .
$$

The derivatives of the water vapor continuity equations [cf. Equations (A7)-(A9)] with respect to the parameter $\alpha^{(13)} \equiv a_{0}$ are as follows:

$$
\begin{gathered}
\frac{\partial N_{3}^{(i)}}{\partial \alpha^{(13)}}=\frac{\partial N_{3}^{(i)}}{\partial a_{0}} \equiv a_{3}^{i, 13}=0 ; \quad \ell=3 ; i=1, \ldots, I-1 ; j=13, \\
\frac{\partial N_{3}^{(I)}}{\partial \alpha^{(13)}}=\frac{\partial N_{3}^{(I)}}{\partial a_{0}} \equiv a_{3}^{I, 13}=\frac{\partial \omega_{i n}}{\partial a_{0}} ; \quad \ell=3 ; i=I ; j=13,
\end{gathered}
$$

where:

$$
\frac{\partial \omega_{i n}}{\partial a_{0}}=\frac{0.622 P_{a t m} \mathrm{e}^{a_{0}+\frac{a_{1}}{T_{d p}}}}{\left(P_{a t m}-\mathrm{e}^{a_{0}+\frac{a_{1}}{T_{d p}}}\right)^{2}} ;
$$

The derivatives of the water vapor continuity equations [cf. Equations (A7)-(A9)] with respect to the parameter $\alpha^{(14)} \equiv a_{1}$ are as follows:

$$
\begin{gathered}
\frac{\partial N_{3}^{(i)}}{\partial \alpha^{(14)}}=\frac{\partial N_{3}^{(i)}}{\partial a_{1}} \equiv a_{3}^{i, 14}=0 ; \quad \ell=3 ; i=1, \ldots, I-1 ; j=14, \\
\frac{\partial N_{3}^{(I)}}{\partial \alpha^{(14)}}=\frac{\partial N_{3}^{(I)}}{\partial a_{1}} \equiv a_{3}^{I, 14}=\frac{\partial \omega_{i n}}{\partial a_{1}} ; \quad \ell=3 ; i=I ; j=14,
\end{gathered}
$$


where:

$$
\frac{\partial \omega_{i n}}{\partial a_{1}}=\frac{0.622 P_{a t m} \mathrm{e}^{a_{0}+\frac{a_{1}}{T_{d p}}}}{T_{d p}\left(P_{a t m}-\mathrm{e}^{a_{0}+\frac{a_{1}}{T_{d p}}}\right)^{2}} .
$$

The derivatives of the water vapor continuity equations [cf. Equations (A7)-(A9)] with respect to the parameter $\alpha^{(15)} \equiv a_{0, \text { cpa }}$ are as follows:

$$
\frac{\partial N_{3}^{(i)}}{\partial \alpha^{(15)}}=\frac{\partial N_{3}^{(i)}}{\partial a_{0, c p a}} \equiv a_{3}^{i, 15}=0 ; \quad \ell=3 ; i=1, \ldots, I ; j=15 .
$$

The derivatives of the water vapor continuity equations [cf. Equations (A7)-(A9)] with respect to the parameter $\alpha^{(16)} \equiv a_{1, \text { cpa }}$ are as follows:

$$
\frac{\partial N_{3}^{(i)}}{\partial \alpha^{(16)}}=\frac{\partial N_{3}^{(i)}}{\partial a_{1, c p a}} \equiv a_{3}^{i, 16}=0 ; \quad \ell=3 ; i=1, \ldots, I ; j=16 .
$$

The derivatives of the water vapor continuity equations [cf. Equations (A7)-(A9)] with respect to the parameter $\alpha^{(17)} \equiv a_{2, \text { cpa }}$ are as follows:

$$
\frac{\partial N_{3}^{(i)}}{\partial \alpha^{(17)}}=\frac{\partial N_{3}^{(i)}}{\partial a_{2, c p a}} \equiv a_{3}^{i, 17}=0 ; \quad \ell=3 ; i=1, \ldots, I ; j=17 .
$$

The derivatives of the water vapor continuity equations [cf. Equations (A7)-(A9)] with respect to the parameter $\alpha^{(18)} \equiv a_{0, \text { dav }}$ are as follows:

$$
\frac{\partial N_{3}^{(i)}}{\partial \alpha^{(18)}}=\frac{\partial N_{3}^{(i)}}{\partial a_{0, \text { dav }}} \equiv a_{3}^{i, 18}=0 ; \quad \ell=3 ; i=1, \ldots, I ; j=18 .
$$

The derivatives of the water vapor continuity equations [cf. Equations (A7)-(A9)] with respect to the parameter $\alpha^{(19)} \equiv a_{1, d a v}$ are as follows:

$$
\frac{\partial N_{3}^{(i)}}{\partial \alpha^{(19)}}=\frac{\partial N_{3}^{(i)}}{\partial a_{1, \text { dav }}} \equiv a_{3}^{i, 19}=0 ; \quad \ell=3 ; i=1, \ldots, I ; j=19 .
$$

The derivatives of the water vapor continuity equations [cf. Equations (A7)-(A9)] with respect to the parameter $\alpha^{(20)} \equiv a_{2, d a v}$ are as follows:

$$
\frac{\partial N_{3}^{(i)}}{\partial \alpha^{(20)}}=\frac{\partial N_{3}^{(i)}}{\partial a_{2, d a v}} \equiv a_{3}^{i, 20}=0 ; \quad \ell=3 ; i=1, \ldots, I ; j=20 .
$$

The derivatives of the water vapor continuity equations [cf. Equations (A7)-(A9)] with respect to the parameter $\alpha^{(21)} \equiv a_{3, \text { dav }}$ are as follows:

$$
\frac{\partial N_{3}^{(i)}}{\partial \alpha^{(21)}}=\frac{\partial N_{3}^{(i)}}{\partial a_{3, d a v}} \equiv a_{3}^{i, 21}=0 ; \quad \ell=3 ; i=1, \ldots, I ; j=21 .
$$

The derivatives of the water vapor continuity equations [cf. Equations (A7)-(A9)] with respect to the parameter $\alpha^{(22)} \equiv a_{0 f}$ are as follows:

$$
\frac{\partial N_{3}^{(i)}}{\partial \alpha^{(22)}}=\frac{\partial N_{3}^{(i)}}{\partial a_{0 f}} \equiv a_{3}^{i, 22}=0 ; \quad \ell=3 ; i=1, \ldots, I ; j=22 .
$$


The derivatives of the water vapor continuity equations [cf. Equations (A7)-(A9)] with respect to the parameter $\alpha^{(23)} \equiv a_{1 f}$ are as follows:

$$
\frac{\partial N_{3}^{(i)}}{\partial \alpha^{(23)}}=\frac{\partial N_{3}^{(i)}}{\partial a_{1 f}} \equiv a_{3}^{i, 23}=0 ; \quad \ell=3 ; i=1, \ldots, I ; j=23 .
$$

The derivatives of the water vapor continuity equations [cf. Equations (A7)-(A9)] with respect to the parameter $\alpha^{(24)} \equiv a_{0 g}$ are as follows:

$$
\frac{\partial N_{3}^{(i)}}{\partial \alpha^{(24)}}=\frac{\partial N_{3}^{(i)}}{\partial a_{0 g}} \equiv a_{3}^{i, 24}=0 ; \quad \ell=3 ; i=1, \ldots, I ; j=24 .
$$

The derivatives of the water vapor continuity equations [cf. Equations (A7)-(A9)] with respect to the parameter $\alpha^{(25)} \equiv a_{1 g}$ are as follows:

$$
\frac{\partial N_{3}^{(i)}}{\partial \alpha^{(25)}}=\frac{\partial N_{3}^{(i)}}{\partial a_{1 g}} \equiv a_{3}^{i, 25}=0 ; \quad \ell=3 ; i=1, \ldots, I ; j=25 .
$$

The derivatives of the water vapor continuity equations [cf. Equations (A7)-(A9)] with respect to the parameter $\alpha^{(26)} \equiv a_{0, N u}$ are as follows:

$$
\frac{\partial N_{3}^{(i)}}{\partial \alpha^{(26)}}=\frac{\partial N_{3}^{(i)}}{\partial a_{0, N u}} \equiv a_{3}^{i, 26}=0 ; \quad \ell=3 ; i=1, \ldots, I ; j=26 .
$$

The derivatives of the water vapor continuity equations [cf. Equations (A7)-(A9)] with respect to the parameter $\alpha^{(27)} \equiv a_{1, N u}$ are as follows:

$$
\frac{\partial N_{3}^{(i)}}{\partial \alpha^{(27)}}=\frac{\partial N_{3}^{(i)}}{\partial a_{1, N u}} \equiv a_{3}^{i, 27}=0 ; \quad \ell=3 ; i=1, \ldots, I ; j=27 .
$$

The derivatives of the water vapor continuity equations [cf. Equations (A7)-(A9)] with respect to the parameter $\alpha^{(28)} \equiv a_{2, N u}$ are as follows:

$$
\frac{\partial N_{3}^{(i)}}{\partial \alpha^{(28)}}=\frac{\partial N_{3}^{(i)}}{\partial a_{2, N u}} \equiv a_{3}^{i, 28}=0 ; \quad \ell=3 ; i=1, \ldots, I ; j=28 .
$$

The derivatives of the water vapor continuity equations [cf. Equations (A7)-(A9)] with respect to the parameter $\alpha^{(29)} \equiv a_{3, N u}$ are as follows:

$$
\frac{\partial N_{3}^{(i)}}{\partial \alpha^{(29)}}=\frac{\partial N_{3}^{(i)}}{\partial a_{3, N u}} \equiv a_{3}^{i, 29}=0 ; \quad \ell=3 ; i=1, \ldots, I ; j=29 .
$$

The derivatives of the water vapor continuity equations [cf. Equations (A7)-(A9)] with respect to the parameter $\alpha^{(30)} \equiv W_{d k x}$ are as follows:

$$
\frac{\partial N_{3}^{(i)}}{\partial \alpha^{(30)}}=\frac{\partial N_{3}^{(i)}}{\partial W_{d k x}} \equiv a_{3}^{i, 30}=0 ; \quad \ell=3 ; i=1, \ldots, I ; j=30 .
$$

The derivatives of the water vapor continuity equations [cf. Equations (A7)-(A9)] with respect to the parameter $\alpha^{(31)} \equiv W_{d k y}$ are as follows:

$$
\frac{\partial N_{3}^{(i)}}{\partial \alpha^{(31)}}=\frac{\partial N_{3}^{(i)}}{\partial W_{d k y}} \equiv a_{3}^{i, 31}=0 ; \quad \ell=3 ; i=1, \ldots, I ; j=31 .
$$


The derivatives of the water vapor continuity equations [cf. Equations (A7)-(A9)] with respect to the parameter $\alpha^{(32)} \equiv \Delta z_{d k}$ are as follows:

$$
\frac{\partial N_{3}^{(i)}}{\partial \alpha^{(32)}}=\frac{\partial N_{3}^{(i)}}{\partial \Delta z_{d k}} \equiv a_{3}^{i, 32}=0 ; \quad \ell=3 ; i=1, \ldots, I ; j=32 .
$$

The derivatives of the water vapor continuity equations [cf. Equations (A7)-(A9)] with respect to the parameter $\alpha^{(33)} \equiv \Delta z_{\text {fan }}$ are as follows:

$$
\frac{\partial N_{3}^{(i)}}{\partial \alpha^{(33)}}=\frac{\partial N_{3}^{(i)}}{\partial \Delta z_{f a n}} \equiv a_{3}^{i, 33}=0 ; \quad \ell=3 ; i=1, \ldots, I ; j=33 .
$$

The derivatives of the water vapor continuity equations [cf. Equations (A7)-(A9)] with respect to the parameter $\alpha^{(34)} \equiv D_{f a n}$ are as follows:

$$
\frac{\partial N_{3}^{(i)}}{\partial \alpha^{(34)}}=\frac{\partial N_{3}^{(i)}}{\partial D_{f a n}} \equiv a_{3}^{i, 34}=-\frac{m_{w}^{(i)}-m_{w}^{(i+1)}}{m_{a}^{2}} \frac{\partial m_{a}}{\partial D_{f a n}} ; \quad \ell=3 ; i=1, \ldots, I ; j=34,
$$

where $\frac{\partial m_{a}}{\partial D_{f a n}}$ was defined in Equation (A71).

The derivatives of the water vapor continuity equations [cf. Equations (A7)-(A9)] with respect to the parameter $\alpha^{(35)} \equiv \Delta z_{\text {fill }}$ are as follows:

$$
\frac{\partial N_{3}^{(i)}}{\partial \alpha^{(35)}}=\frac{\partial N_{3}^{(i)}}{\partial \Delta z_{f i l l}} \equiv a_{3}^{i, 35}=0 ; \quad \ell=3 ; i=1, \ldots, I ; j=35 .
$$

The derivatives of the water vapor continuity equations [cf. Equations (A7)-(A9)] with respect to the parameter $\alpha^{(36)} \equiv \Delta z_{\text {rain }}$ are as follows:

$$
\frac{\partial N_{3}^{(i)}}{\partial \alpha^{(36)}}=\frac{\partial N_{3}^{(i)}}{\partial \Delta z_{\text {rain }}} \equiv a_{3}^{i, 36}=0 ; \quad \ell=3 ; i=1, \ldots, I ; j=36 .
$$

The derivatives of the water vapor continuity equations [cf. Equations (A7)-(A9)] with respect to the parameter $\alpha^{(37)} \equiv \Delta z_{b s}$ are as follows:

$$
\frac{\partial N_{3}^{(i)}}{\partial \alpha^{(37)}}=\frac{\partial N_{3}^{(i)}}{\partial \Delta z_{b s}} \equiv a_{3}^{i, 37}=0 ; \quad \ell=3 ; i=1, \ldots, I ; j=37 .
$$

The derivatives of the water vapor continuity equations [cf. Equations (A7)-(A9)] with respect to the parameter $\alpha^{(38)} \equiv \Delta z_{d e}$ are as follows:

$$
\frac{\partial N_{3}^{(i)}}{\partial \alpha^{(38)}}=\frac{\partial N_{3}^{(i)}}{\partial \Delta z_{d e}} \equiv a_{3}^{i, 38}=0 ; \quad \ell=3 ; i=1, \ldots, I ; j=38 .
$$

The derivatives of the water vapor continuity equations [cf. Equations (A7)-(A9)] with respect to the parameter $\alpha^{(39)} \equiv D_{h}$ are as follows:

$$
\frac{\partial N_{3}^{(i)}}{\partial \alpha^{(39)}}=\frac{\partial N_{3}^{(i)}}{\partial D_{h}} \equiv a_{3}^{i, 39}=0 ; \quad \ell=3 ; i=1, \ldots, I ; j=39 .
$$


The derivatives of the water vapor continuity equations [cf. Equations (A7)-(A9)] with respect to the parameter $\alpha^{(40)} \equiv A_{\text {fill }}$ are as follows:

$$
\frac{\partial N_{3}^{(i)}}{\partial \alpha^{(40)}}=\frac{\partial N_{3}^{(i)}}{\partial A_{\text {fill }}} \equiv a_{3}^{i, 40}=0 ; \quad \ell=3 ; i=1, \ldots, I ; j=40 .
$$

The derivatives of the water vapor continuity equations [cf. Equations (A7)-(A9)] with respect to the parameter $\alpha^{(41)} \equiv A_{\text {surf }}$ are as follows:

$$
\frac{\partial N_{3}^{(i)}}{\partial \alpha^{(41)}}=\frac{\partial N_{3}^{(i)}}{\partial A_{\text {surf }}} \equiv a_{3}^{i, 41}=0 ; \quad \ell=3 ; i=1, \ldots, I ; j=41 .
$$

The derivatives of the water vapor continuity equations [cf. Equations (A7)-(A9)] with respect to the parameter $\alpha^{(42)} \equiv \operatorname{Pr}$ are as follows:

$$
\frac{\partial N_{3}^{(i)}}{\partial \alpha^{(42)}}=\frac{\partial N_{3}^{(i)}}{\partial \operatorname{Pr}} \equiv a_{3}^{i, 42}=0 ; \quad \ell=3 ; i=1, \ldots, I ; j=42 .
$$

The derivatives of the water vapor continuity equations [cf. Equations (A7)-(A9)] with respect to the parameter $\alpha^{(43)} \equiv V_{w}$ are as follows:

$$
\frac{\partial N_{3}^{(i)}}{\partial \alpha^{(43)}}=\frac{\partial N_{3}^{(i)}}{\partial V_{w}} \equiv a_{3}^{i, 43}=0 ; \quad \ell=3 ; i=1, \ldots, I ; j=43 .
$$

The derivatives of the water vapor continuity equations [cf. Equations (A7)-(A9)] with respect to the parameter $\alpha^{(44)} \equiv V_{\text {exit }}$ are as follows:

$$
\frac{\partial N_{3}^{(i)}}{\partial \alpha^{(44)}}=\frac{\partial N_{3}^{(i)}}{\partial V_{\text {exit }}} \equiv a_{3}^{i, 44}=-\frac{m_{w}^{(i)}-m_{w}^{(i+1)}}{m_{a}^{2}} \frac{\partial m_{a}}{\partial V_{\text {exit }}} ; \quad \ell=3 ; i=1, \ldots, I ; j=44,
$$

where $\frac{\partial m_{a}}{\partial V_{\text {exit }}}$ was defined in Equation (A86).

The derivatives of the water vapor continuity equations [cf. Equations (A7)-(A9)] with respect to the parameter $\alpha^{(45)} \equiv m_{w, \text { in }}$ are as follows:

$$
\begin{gathered}
\frac{\partial N_{3}^{(1)}}{\partial \alpha^{(45)}}=\frac{\partial N_{3}^{(1)}}{\partial m_{w, i n}} \equiv a_{3}^{1,45}=\frac{1}{m_{a}} ; \quad \ell=3 ; i=1 ; j=45, \\
\frac{\partial N_{3}^{(i)}}{\partial \alpha^{(45)}}=\frac{\partial N_{3}^{(i)}}{\partial m_{w, i n}} \equiv a_{3}^{i, 45}=0 ; \quad \ell=3 ; i=2, \ldots, I ; j=45 .
\end{gathered}
$$

The derivatives of the water vapor continuity equations [cf. Equations (A7)-(A9)] with respect to the parameter $\alpha^{(46)} \equiv T_{a, i n}$ are as follows:

$$
\frac{\partial N_{3}^{(i)}}{\partial \alpha(46)}=\frac{\partial N_{3}^{(i)}}{\partial T_{a, i n}} \equiv a_{3}^{i, 46}=0 ; \quad \ell=3 ; i=1, \ldots, I ; j=46 .
$$

The derivatives of the water vapor continuity equations [cf. Equations (A7)-(A9)] with respect to the parameter $\alpha^{(47)} \equiv m_{a}$ are as follows:

$$
\frac{\partial N_{3}^{(i)}}{\partial \alpha^{(47)}}=\frac{\partial N_{3}^{(i)}}{\partial m_{a}} \equiv a_{3}^{i, 47}=-\frac{m_{w}^{(i)}-m_{w}^{(i+1)}}{m_{a}^{2}} ; \quad \ell=3 ; i=1, \ldots, I ; j=47 .
$$


The derivatives of the water vapor continuity equations [cf. Equations (A7)-(A9)] with respect to the parameter $\alpha^{(48)} \equiv \omega_{\text {in }}$ are as follows:

$$
\begin{gathered}
\frac{\partial N_{3}^{(i)}}{\partial \alpha^{(48)}}=\frac{\partial N_{3}^{(i)}}{\partial \omega_{i n}} \equiv a_{3}^{i, 48}=0 ; \quad \ell=3 ; i=1, \ldots, I-1 ; j=48, \\
\frac{\partial N_{3}^{(I)}}{\partial \alpha^{(48)}}=\frac{\partial N_{3}^{(I)}}{\partial \omega_{\text {in }}} \equiv a_{3}^{I, 48}=1 ; \quad \ell=3 ; i=I ; j=48 .
\end{gathered}
$$

The derivatives of the water vapor continuity equations [cf. Equations (A7)-(A9)] with respect to the parameter $\alpha^{(49)} \equiv \operatorname{Re}_{d}$ are as follows:

$$
\frac{\partial N_{3}^{(i)}}{\partial \alpha^{(49)}}=\frac{\partial N_{3}^{(i)}}{\partial \operatorname{Re}_{d}} \equiv a_{3}^{i, 49}=0 ; \quad \ell=3 ; i=1, \ldots, I ; j=49 .
$$

The derivatives of the water vapor continuity equations [cf. Equations (A7)-(A9)] with respect to the parameter $\alpha^{(50)} \equiv$ Sc are as follows:

$$
\frac{\partial N_{3}^{(i)}}{\partial \alpha^{(50)}}=\frac{\partial N_{3}^{(i)}}{\partial S c} \equiv a_{3}^{i, 50}=0 ; \quad \ell=3 ; i=1, \ldots, I ; j=50 .
$$

The derivatives of the water vapor continuity equations [cf. Equations (A7)-(A9)] with respect to the parameter $\alpha^{(51)} \equiv S h$ are as follows:

$$
\frac{\partial N_{3}^{(i)}}{\partial \alpha^{(51)}}=\frac{\partial N_{3}^{(i)}}{\partial S h} \equiv a_{3}^{i, 51}=0 ; \ell=3 ; i=1, \ldots, I ; j=51 .
$$

The derivatives of the water vapor continuity equations [cf. Equations (A7)-(A9)] with respect to the parameter $\alpha^{(52)} \equiv N u$ are as follows:

$$
\frac{\partial N_{3}^{(i)}}{\partial \alpha^{(52)}}=\frac{\partial N_{3}^{(i)}}{\partial N u} \equiv a_{3}^{i, 52}=0 ; \quad \ell=3 ; i=1, \ldots, I ; j=52 .
$$

A4. Derivatives of the Air and Water Vapor Energy Balance Equations with Respect to the Parameters

The derivatives of the air/water vapor energy balance equations [cf. Equations (A10)-(A12)] with respect to the parameter $\alpha^{(1)} \equiv T_{d b}$ are as follows:

$$
\begin{gathered}
\frac{\partial N_{4}^{(i)}}{\partial \alpha^{(1)}}=\frac{\partial N_{4}^{(i)}}{\partial T_{d b}} \equiv a_{4}^{i, 1}=0 ; \quad \ell=4 ; i=1, \ldots, I-1 ; j=1, \\
\frac{\partial N_{4}^{(I)}}{\partial \alpha^{(1)}}=\frac{\partial N_{4}^{(I)}}{\partial T_{d b}} \equiv a_{4}^{I, 1}=C_{p}^{(I)}\left(\frac{T_{a}^{(I)}+273.15}{2}, \alpha\right)+\omega_{i n} \frac{\partial h_{g, a}^{(I+1)}\left(T_{a, i n}, \alpha\right)}{\partial T_{a, i n}} \\
=C_{p}^{(I)}\left(\frac{T_{a}^{(I)}+273.15}{2}, \alpha\right)+\omega_{i n} a_{1 g} ; \quad \ell=4 ; i=I ; j=1 .
\end{gathered}
$$

Note: The value of the inlet air temperature is set equal to dry-bulb temperature, although these quantities are treated as two different parameters in the model. The dry-bulb temperature is used in mass diffusivity calculations. The relation between the two parameters, i.e., $T_{a, i n}=T_{d b}$, needs to be accounted for when computing the respective derivatives: the derivative of Equation (A12) with respect to the dry-bulb temperature must be the same as the derivative of Equation (A12) with respect to the inlet air temperature. 
The derivatives of the air/water vapor energy balance equations [cf. Equations (A10)-(A12)] with respect to the parameter $\alpha^{(2)} \equiv T_{d p}$ are as follows:

$$
\begin{gathered}
\frac{\partial N_{4}^{(i)}}{\partial \alpha^{(2)}}=\frac{\partial N_{4}^{(i)}}{\partial T_{d p}} \equiv a_{4}^{i, 2}=0 ; \quad \ell=4 ; i=1, \ldots, I-1 ; j=2, \\
\frac{\partial N_{4}^{(I)}}{\partial \alpha^{(2)}}=\frac{\partial N_{4}^{(I)}}{\partial T_{d p}} \equiv a_{4}^{I, 2}=\frac{\partial \omega_{i n}}{\partial T_{d p}}\left(a_{1 g} T_{a, i n}+a_{0 g}\right) ; \quad \ell=4 ; i=I ; j=2,
\end{gathered}
$$

where $\frac{\partial \omega_{i n}}{\partial T_{d p}}$ was defined in Equation (A166).

The derivatives of the air/water vapor energy balance equations [cf. Equations (A10)-(A12)] with respect to the parameter $\alpha^{(3)} \equiv T_{w, \text { in }}$ are as follows:

$$
\begin{aligned}
\frac{\partial N_{4}^{(1)}}{\partial \alpha^{(3)}}=\frac{\partial N_{4}^{(1)}}{\partial T_{w, i n}} \equiv a_{4}^{1,3} & =\frac{h_{g, w}^{(2)}\left(T_{w w}^{(2)}, \alpha\right)}{m_{a}} \frac{\partial m_{w, i n}}{\partial T_{w, i n}}=\frac{a_{1 g} T_{w w}^{(2)}+a_{0 g}}{m_{a}} \frac{\partial m_{w, i n}}{\partial T_{w, i n}} ; \\
\ell & =4 ; i=1 ; j=3,
\end{aligned}
$$

where $\frac{\partial \omega_{w, i n}}{\partial T_{w, i n}}$ was defined in Equation (A20), and

$$
\frac{\partial N_{4}^{(i)}}{\partial \alpha^{(3)}}=\frac{\partial N_{4}^{(i)}}{\partial T_{w, i n}} \equiv a_{4}^{i, 3}=0 ; \quad \ell=4 ; i=2, \ldots, I ; j=3 .
$$

The derivatives of the air/water vapor energy balance equations [cf. Equations (A10)-(A12)] with respect to the parameter $\alpha^{(4)} \equiv P_{\text {atm }}$ are as follows:

$$
\begin{aligned}
& \frac{\partial N_{4}^{(i)}}{\partial \alpha^{(4)}}=\frac{\partial N_{4}^{(i)}}{\partial P_{a t m}} \equiv a_{4}^{i, 4}=\left(T_{w}^{(i+1)}-T_{a}^{(i)}\right)\left[\frac{1}{m_{a}} \frac{\partial H\left(m_{a}, \alpha\right)}{\partial N u(R e, \alpha)} \frac{\partial N u(\operatorname{Re}, \alpha)}{\partial m_{a}} \frac{\partial m_{a}}{\partial P_{a t m}}-\frac{H\left(m_{a}, \alpha\right)}{m_{a}{ }^{2}} \frac{\partial m_{a}}{\partial P_{a t m}}\right] \\
& -\frac{\left(m_{w}^{(i)}-m_{w}^{(i+1)}\right) h_{g, w}^{(i+1)}\left(T_{w}^{(i+1)}, \alpha\right)}{m_{a}{ }^{2}} \frac{\partial m_{a}}{\partial P_{a t m}} ; \quad \ell=4 ; i=1, \ldots, I-1 ; j=4 \text {, } \\
& \frac{\partial N_{4}^{(I)}}{\partial \alpha^{(4)}}=\frac{\partial N_{4}^{(I)}}{\partial P_{a t m}} \equiv a_{4}^{I, 4}=\left(T_{w}^{(i+1)}-T_{a}^{(i)}\right)\left[\frac{1}{m_{a}} \frac{\partial H\left(m_{a}, \alpha\right)}{\partial N u(\operatorname{Re}, \boldsymbol{\alpha})} \frac{\partial N u(\operatorname{Re}, \boldsymbol{\alpha})}{\partial m_{a}} \frac{\partial m_{a}}{\partial P_{a t m}}-\frac{H\left(m_{a}, \alpha\right)}{m_{a}{ }^{2}} \frac{\partial m_{a}}{\partial P_{a t m}}\right] \\
& -\frac{\left(m_{w}^{(i)}-m_{w}^{(i+1)}\right) h_{g, w}^{(i+1)}\left(T_{w}^{(i+1)}, \alpha\right)}{m_{a}{ }^{2}} \frac{\partial m_{a}}{\partial P_{a t m}}+\frac{\partial \omega_{i n}}{\partial P_{a t m}}\left(a_{1 g} T_{a, i n}+a_{0 g}\right) ; \quad \ell=4 ; i=I ; j=4,
\end{aligned}
$$

where $\frac{\partial H\left(m_{a}, \boldsymbol{\alpha}\right)}{\partial N u(\operatorname{Re}, \boldsymbol{\alpha})}$ and $\frac{\partial N u(\operatorname{Re}, \boldsymbol{\alpha})}{\partial m_{a}}$ were defined in Equations (A24) and (A105), respectively, while $\frac{\partial m_{a}}{\partial P_{a t m}}$ and $\frac{\partial \omega_{i n}}{\partial P_{a t m}}$ were defined in Equations (A25) and (A171), respectively.

The derivatives of the air/water vapor energy balance equations [cf. Equations (A10)-(A12)] with respect to the parameter $\alpha^{(5)} \equiv w_{t s a}$ are as follows:

$$
\frac{\partial N_{4}^{(i)}}{\partial \alpha^{(5)}}=\frac{\partial N_{4}^{(i)}}{\partial w_{t s a}} \equiv a_{4}^{i, 5}=\frac{\left(T_{w}^{(i+1)}-T_{a}^{(i)}\right)}{m_{a}} \frac{\partial H\left(m_{a}, \alpha\right)}{\partial w_{t s a}} ; \quad \ell=4 ; i=1, \ldots, I ; j=5,
$$

where $\frac{\partial H\left(m_{a}, \alpha\right)}{\partial w_{t s a}}$ was defined in Equation (A107).

The derivatives of the air/water vapor energy balance equations [cf. Equations (A10)-(A12)] with respect to the parameter $\alpha^{(6)} \equiv k_{\text {sum }}$ are as follows:

$$
\frac{\partial N_{4}^{(i)}}{\partial \alpha^{(6)}}=\frac{\partial N_{4}^{(i)}}{\partial k_{\text {sum }}} \equiv a_{4}^{i, 6}=0 ; \quad \ell=4 ; i=1, \ldots, I ; j=6 .
$$

The derivatives of the air/water vapor energy balance equations [cf. Equations (A10)-(A12)] with respect to the parameter $\alpha^{(7)} \equiv \mu$ are as follows:

$$
\frac{\partial N_{4}^{(i)}}{\partial \alpha^{(7)}}=\frac{\partial N_{4}^{(i)}}{\partial \mu} \equiv a_{4}^{i, 7}=\frac{\left(T_{w}^{(i+1)}-T_{a}^{(i)}\right)}{m_{a}} \frac{\partial H\left(m_{a}, \alpha\right)}{\partial \mu} ; \quad \ell=4 ; i=1, \ldots, I ; j=7,
$$


where $\frac{\partial H\left(m_{a}, \boldsymbol{\alpha}\right)}{\partial}$ was defined in Equation (A110).

The derivatives of the air/water vapor energy balance equations [cf. Equations (A10)-(A12)] with respect to the parameter $\alpha^{(8)} \equiv v$ are as follows:

$$
\frac{\partial N_{4}^{(i)}}{\partial \alpha^{(8)}}=\frac{\partial N_{4}^{(i)}}{\partial v} \equiv a_{4}^{i, 8}=0 ; \quad \ell=4 ; i=1, \ldots, I ; j=8 .
$$

The derivatives of the air/water vapor energy balance equations [cf. Equations (A10)-(A12)] with respect to the parameter $\alpha^{(9)} \equiv k_{\text {air }}$ are as follows:

$$
\frac{\partial N_{4}^{(i)}}{\partial \alpha^{(9)}}=\frac{\partial N_{4}^{(i)}}{\partial k_{\text {air }}} \equiv a_{4}^{i, 9}=\frac{\left(T_{w}^{(i+1)}-T_{a}^{(i)}\right)}{m_{a}} \frac{\partial H\left(m_{a}, \alpha\right)}{\partial k_{a i r}} ; \quad \ell=4 ; i=1, \ldots, I ; j=9,
$$

where $\frac{\partial H\left(m_{a}, \alpha\right)}{\partial k_{a i r}}$ was defined in Equation (A113).

The derivatives of the air/water vapor energy balance equations [cf. Equations (A10)-(A12)] with respect to the parameter $\alpha^{(10)} \equiv f_{h t}$ are as follows:

$$
\frac{\partial N_{4}^{(i)}}{\partial \alpha^{(10)}}=\frac{\partial N_{4}^{(i)}}{\partial f_{h t}} \equiv a_{4}^{i, 10}=\frac{\left(T_{w}^{(i+1)}-T_{a}^{(i)}\right)}{m_{a}} \frac{\partial H\left(m_{a}, \boldsymbol{\alpha}\right)}{\partial f_{h t}} ; \quad \ell=4 ; i=1, \ldots, I ; j=10,
$$

where $\frac{\partial H\left(m_{a}, \alpha\right)}{\partial f_{h t}}$ was defined in Equation (A115).

The derivatives of the air/water vapor energy balance equations [cf. Equations (A10)-(A12)] with respect to the parameter $\alpha^{(11)} \equiv f_{m t}$ are as follows:

$$
\frac{\partial N_{4}^{(i)}}{\partial \alpha^{(11)}}=\frac{\partial N_{4}^{(i)}}{\partial f_{m t}} \equiv a_{4}^{i, 11}=0 ; \quad \ell=4 ; i=1, \ldots, I ; j=11 .
$$

The derivatives of the air/water vapor energy balance equations [cf. Equations (A10)-(A12)] with respect to the parameter $\alpha^{(12)} \equiv f$ are as follows:

$$
\frac{\partial N_{4}^{(i)}}{\partial \alpha^{(12)}}=\frac{\partial N_{4}^{(i)}}{\partial f} \equiv a_{4}^{i, 12}=0 ; \quad \ell=4 ; i=1, \ldots, I ; j=12 .
$$

The derivatives of the air/water vapor energy balance equations [cf. Equations (A10)-(A12)] with respect to the parameter $\alpha^{(13)} \equiv a_{0}$ are as follows:

$$
\begin{gathered}
\frac{\partial N_{4}^{(i)}}{\partial \alpha^{(13)}}=\frac{\partial N_{4}^{(i)}}{\partial a_{0}} \equiv a_{4}^{i, 13}=0 ; \quad \ell=4 ; i=1, \ldots, I-1 ; j=13, \\
\frac{\partial N_{4}^{(I)}}{\partial \alpha^{(13)}}=\frac{\partial N_{4}^{(I)}}{\partial a_{0}} \equiv a_{4}^{I, 13}=\frac{\partial \omega_{i n}}{\partial a_{0}}\left(a_{1 g} T_{a, i n}+a_{0 g}\right) ; \quad \ell=4 ; i=I ; j=13,
\end{gathered}
$$

where $\frac{\partial \omega_{i n}}{\partial a_{0}}$ was defined in Equation (A182).

The derivatives of the air/water vapor energy balance equations [cf. Equations (A10)-(A12)] with respect to the parameter $\alpha^{(14)} \equiv a_{1}$ are as follows:

$$
\begin{gathered}
\frac{\partial N_{4}^{(i)}}{\partial \alpha^{(14)}}=\frac{\partial N_{4}^{(i)}}{\partial a_{1}} \equiv a_{4}^{i, 14}=0 ; \quad \ell=4 ; i=1, \ldots, I-1 ; j=14, \\
\frac{\partial N_{4}^{(I)}}{\partial \alpha^{(14)}}=\frac{\partial N_{4}^{(I)}}{\partial a_{1}} \equiv a_{4}^{I, 14}=\frac{\partial \omega_{i n}}{\partial a_{1}}\left(a_{1 g} T_{a, i n}+a_{0 g}\right) ; \quad \ell=4 ; i=I ; j=14,
\end{gathered}
$$

where $\frac{\partial \omega_{i n}}{\partial a_{1}}$ was defined in Equation (A185). 
The derivatives of the air/water vapor energy balance equations [cf. Equations (A10)-(A12)] with respect to the parameter $\alpha^{(15)} \equiv a_{0, c p a}$ are as follows:

$$
\begin{gathered}
\frac{\partial N_{4}^{(i)}}{\partial \alpha^{(15)}}=\frac{\partial N_{4}^{(i)}}{\partial a_{0, c p a}} \equiv a_{4}^{i, 15}=\left(T_{a}^{(i+1)}-T_{a}^{(i)}\right) \frac{\partial C_{p}^{(i)}\left(\frac{T_{a}^{(i)}+273.15}{2}, \alpha\right)}{\partial a_{0, c p a}}=T_{a}^{(i+1)}-T_{a}^{(i)} ; \\
\ell=4 ; i=1, \ldots, I ; j=15 .
\end{gathered}
$$

The derivatives of the air/water vapor energy balance equations [cf. Equations (A10)-(A12)] with respect to the parameter $\alpha^{(16)} \equiv a_{1, c p a}$ are as follows:

$$
\begin{gathered}
\frac{\partial N_{4}^{(i)}}{\partial \alpha^{(16)}} \quad=\frac{\partial N_{4}^{(i)}}{\partial a_{1, c p a}} \equiv a_{4}^{i, 16}=\left(T_{a}^{(i+1)}-T_{a}^{(i)}\right) \frac{\partial C_{p}^{(i)}\left(\frac{T_{a}^{(i)}+273.15}{2}, \alpha\right)}{\partial a_{1, c p a}} \\
=0.5\left(T_{a}^{(i+1)}-T_{a}^{(i)}\right)\left(T_{a}^{(i)}+273.15\right) ; \quad \ell=4 ; i=1, \ldots, I ; j=16 .
\end{gathered}
$$

The derivatives of the air/water vapor energy balance equations [cf. Equations (A10)-(A12)] with respect to the parameter $\alpha^{(17)} \equiv a_{2, c p a}$ are as follows:

$$
\begin{gathered}
\frac{\partial N_{4}^{(i)}}{\partial \alpha^{(17)}} \quad=\frac{\partial N_{4}^{(i)}}{\partial a_{2, c p a}} \equiv a_{4}^{i, 17}=\left(T_{a}^{(i+1)}-T_{a}^{(i)}\right) \frac{\partial C_{p}^{(i)}\left(\frac{T_{a}^{(i)}+273.15}{2}, \alpha\right)}{\partial a_{2, c p a}} \\
=0.25\left(T_{a}^{(i+1)}-T_{a}^{(i)}\right)\left[T_{a}^{(i)}+273.15\right]^{2} ; \quad \ell=4 ; i=1, \ldots, I ; j=17 .
\end{gathered}
$$

The derivatives of the air/water vapor energy balance equations [cf. Equations (A10)-(A12)] with respect to the parameter $\alpha^{(18)} \equiv a_{0, d a v}$ are as follows:

$$
\frac{\partial N_{4}^{(i)}}{\partial \alpha^{(18)}}=\frac{\partial N_{4}^{(i)}}{\partial a_{0, \text { dav }}} \equiv a_{4}^{i, 18}=0 ; \quad \ell=4 ; i=1, \ldots, I ; j=18 .
$$

The derivatives of the air/water vapor energy balance equations [cf. Equations (A10)-(A12)] with respect to the parameter $\alpha^{(19)} \equiv a_{1, d a v}$ are as follows:

$$
\frac{\partial N_{4}^{(i)}}{\partial \alpha^{(19)}}=\frac{\partial N_{4}^{(i)}}{\partial a_{1, \text { dav }}} \equiv a_{4}^{i, 19}=0 ; \quad \ell=4 ; i=1, \ldots, I ; j=19 .
$$

The derivatives of the air/water vapor energy balance equations [cf. Equations (A10)-(A12)] with respect to the parameter $\alpha^{(20)} \equiv a_{2, \text { dav }}$ are as follows:

$$
\frac{\partial N_{4}^{(i)}}{\partial \alpha^{(20)}}=\frac{\partial N_{4}^{(i)}}{\partial a_{2, d a v}} \equiv a_{4}^{i, 20}=0 ; \quad \ell=4 ; i=1, \ldots, I ; j=20 .
$$

The derivatives of the air/water vapor energy balance equations [cf. Equations (A10)-(A12)] with respect to the parameter $\alpha^{(21)} \equiv a_{3, d a v}$ are as follows:

$$
\frac{\partial N_{4}^{(i)}}{\partial \alpha^{(21)}}=\frac{\partial N_{4}^{(i)}}{\partial a_{3, d a v}} \equiv a_{4}^{i, 21}=0 ; \quad \ell=4 ; i=1, \ldots, I ; j=21 .
$$

The derivatives of the air/water vapor energy balance equations [cf. Equations (A10)-(A12)] with respect to the parameter $\alpha^{(22)} \equiv a_{0 f}$ are as follows:

$$
\frac{\partial N_{4}^{(i)}}{\partial \alpha^{(22)}}=\frac{\partial N_{4}^{(i)}}{\partial a_{0 f}} \equiv a_{4}^{i, 22}=0 ; \quad \ell=4 ; i=1, \ldots, I ; j=22 .
$$


The derivatives of the air/water vapor energy balance equations [cf. Equations (A10)-(A12)] with respect to the parameter $\alpha^{(23)} \equiv a_{1 f}$ are as follows:

$$
\frac{\partial N_{4}^{(i)}}{\partial \alpha^{(23)}}=\frac{\partial N_{4}^{(i)}}{\partial a_{1 f}} \equiv a_{4}^{i, 23}=0 ; \quad \ell=4 ; i=1, \ldots, I ; j=23 .
$$

The derivatives of the air/water vapor energy balance equations [cf. Equations (A10)-(A12)] with respect to the parameter $\alpha^{(24)} \equiv a_{0 g}$ are as follows:

$$
\frac{\partial N_{4}^{(i)}}{\partial \alpha^{(24)}}=\frac{\partial N_{4}^{(i)}}{\partial a_{0 g}} \equiv a_{4}^{i, 24}=\omega^{(i+1)}-\omega^{(i)}+\frac{m_{w}^{(i)}-m_{w}^{(i+1)}}{m_{a}} ; \quad \ell=4 ; i=1, \ldots, I ; j=24 .
$$

The derivatives of the air/water vapor energy balance equations [cf. Equations (A10)-(A12)] with respect to the parameter $\alpha^{(25)} \equiv a_{1 g}$ are as follows:

$$
\begin{gathered}
\frac{\partial N_{4}^{(i)}}{\partial \alpha^{(25)}}=\frac{\partial N_{4}^{(i)}}{\partial a_{1 g}} \equiv a_{4}^{i, 25}=\omega^{(i+1)} T_{a}^{(i+1)}-\omega^{(i)} T_{a}^{(i)}+\frac{\left(m_{w}^{(i)}-m_{w}^{(i+1)}\right) T_{w}^{(i+1)}}{m_{a}} ; \\
\ell=4 ; i=1, \ldots, I ; j=25 .
\end{gathered}
$$

The derivatives of the air/water vapor energy balance equations [cf. Equations (A10)-(A12)] with respect to the parameter $\alpha^{(26)} \equiv a_{0, N u}$ are as follows:

$$
\begin{gathered}
\frac{\partial N_{4}^{(i)}}{\partial \alpha^{(26)}}=\frac{\partial N_{4}^{(i)}}{\partial a_{0, N u}} \equiv a_{4}^{i, 26}=\frac{\left(T_{w}^{(i+1)}-T_{a}^{(i)}\right)}{m_{a}} \frac{\partial H\left(m_{a}, \alpha\right)}{\partial N u(\operatorname{Re}, \alpha)} \frac{\partial N u(\operatorname{Re}, \alpha)}{\partial a_{0, N u}} ; \\
\ell=4 ; i=1, \ldots, I ; j=26,
\end{gathered}
$$

where $\frac{\partial H\left(m_{a}, \boldsymbol{\alpha}\right)}{\partial N u(\operatorname{Re}, \boldsymbol{\alpha})}$ and $\frac{\partial N u(\mathrm{Re}, \boldsymbol{\alpha})}{\partial a_{0}, N u}$ were defined previously in Equations (A59) and (A105), respectively.

The derivatives of the air/water vapor energy balance equations [cf. Equations (A10)-(A12)] with respect to the parameter $\alpha^{(27)} \equiv a_{1, N u}$ are as follows:

$$
\begin{gathered}
\frac{\partial N_{4}^{(i)}}{\partial \alpha^{(27)}}=\frac{\partial N_{4}^{(i)}}{\partial a_{1, N u}} \equiv a_{4}^{i, 27}=\frac{\left(T_{w}^{(i+1)}-T_{a}^{(i)}\right)}{m_{a}} \frac{\partial H\left(m_{a}, \alpha\right)}{\partial N u(\operatorname{Re}, \alpha)} \frac{\partial N u(\operatorname{Re}, \alpha)}{\partial a_{1, N u}} ; \\
\ell=4 ; i=1, \ldots, I ; j=27,
\end{gathered}
$$

where $\frac{\partial H\left(m_{a}, \boldsymbol{\alpha}\right)}{\partial N u(\operatorname{Re}, \boldsymbol{\alpha})}$ and $\frac{\partial N u(\mathrm{Re}, \boldsymbol{\alpha})}{\partial a_{1, N u}}$ were defined previously in Equations (A61) and (A105), respectively.

The derivatives of the air/water vapor energy balance equations [cf. Equations (A10)-(A12)] with respect to the parameter $\alpha^{(28)} \equiv a_{2, N u}$ are as follows:

$$
\begin{gathered}
\frac{\partial N_{4}^{(i)}}{\partial \alpha^{(28)}}=\frac{\partial N_{4}^{(i)}}{\partial a_{2, N u}} \equiv a_{4}^{i, 28}=\frac{\left(T_{w}^{(i+1)}-T_{a}^{(i)}\right)}{m_{a}} \frac{\partial H\left(m_{a}, \alpha\right)}{\partial N u(\operatorname{Re}, \boldsymbol{\alpha})} \frac{\partial N u(\mathrm{Re}, \boldsymbol{\alpha})}{\partial a_{2, N u}} ; \\
\ell=4 ; i=1, \ldots, I ; j=28,
\end{gathered}
$$

where $\frac{\partial H\left(m_{a}, \alpha\right)}{\partial N u(\operatorname{Re}, \boldsymbol{\alpha})}$ and $\frac{\partial N u(\mathrm{Re}, \boldsymbol{\alpha})}{\partial a_{2, N u}}$ were defined previously in Equations (A63) and (A105), respectively.

The derivatives of the air/water vapor energy balance equations [cf. Equations (A10)-(A12)] with respect to the parameter $\alpha^{(29)} \equiv a_{3, \mathrm{~N} u}$ are as follows:

$$
\begin{gathered}
\frac{\partial N_{4}^{(i)}}{\partial \alpha^{(29)}}=\frac{\partial N_{4}^{(i)}}{\partial a_{3, N u}} \equiv a_{4}^{i, 29}=\frac{\left(T_{w}^{(i+1)}-T_{a}^{(i)}\right)}{m_{a}} \frac{\partial H\left(m_{a}, \alpha\right)}{\partial N u(\operatorname{Re}, \alpha)} \frac{\partial N u(\mathrm{Re}, \alpha)}{\partial a_{3, N u}} ; \\
\ell=4 ; i=1, \ldots, I ; j=29,
\end{gathered}
$$

where $\frac{\partial H\left(m_{a}, \boldsymbol{\alpha}\right)}{\partial N u(\operatorname{Re}, \boldsymbol{\alpha})}$ and $\frac{\partial N u(\operatorname{Re}, \boldsymbol{\alpha})}{\partial a_{3, N u}}$ were defined previously in Equations (A65) and (A105), respectively. 
The derivatives of the air/water vapor energy balance equations [cf. Equations (A10)-(A12)] with respect to the parameter $\alpha^{(30)} \equiv W_{d k x}$ are as follows:

$$
\frac{\partial N_{4}^{(i)}}{\partial \alpha^{(30)}}=\frac{\partial N_{4}^{(i)}}{\partial W_{d k x}} \equiv a_{4}^{i, 30}=0 ; \quad \ell=4 ; i=1, \ldots, I ; j=30 .
$$

The derivatives of the air/water vapor energy balance equations [cf. Equations (A10)-(A12)] with respect to the parameter $\alpha^{(31)} \equiv W_{d k y}$ are as follows:

$$
\frac{\partial N_{4}^{(i)}}{\partial \alpha^{(31)}}=\frac{\partial N_{4}^{(i)}}{\partial W_{d k y}} \equiv a_{4}^{i, 31}=0 ; \quad \ell=4 ; i=1, \ldots, I ; j=31 .
$$

The derivatives of the air/water vapor energy balance equations [cf. Equations (A10)-(A12)] with respect to the parameter $\alpha^{(32)} \equiv \Delta z_{d k}$ are as follows:

$$
\frac{\partial N_{4}^{(i)}}{\partial \alpha^{(32)}}=\frac{\partial N_{4}^{(i)}}{\partial \Delta z_{d k}} \equiv a_{4}^{i, 32}=0 ; \quad \ell=4 ; i=1, \ldots, I ; j=32 .
$$

The derivatives of the air/water vapor energy balance equations [cf. Equations (A10)-(A12)] with respect to the parameter $\alpha^{(33)} \equiv \Delta z_{f a n}$ are as follows:

$$
\frac{\partial N_{4}^{(i)}}{\partial \alpha^{(33)}}=\frac{\partial N_{4}^{(i)}}{\partial \Delta z_{f a n}} \equiv a_{4}^{i, 33}=0 ; \quad \ell=4 ; i=1, \ldots, I ; j=33 .
$$

The derivatives of the air/water vapor energy balance equations [cf. Equations (A10)-(A12)] with respect to the parameter $\alpha^{(34)} \equiv D_{f a n}$ are as follows:

$$
\begin{gathered}
\frac{\partial N_{4}^{(i)}}{\partial \alpha^{(34)}}=\frac{\partial N_{4}^{(i)}}{\partial D_{f a n}} \equiv a_{4}^{i, 34}=\left(T_{w}^{(i+1)}-T_{a}^{(i)}\right)\left[\frac{1}{m_{a}} \frac{\partial H\left(m_{a}, \alpha\right)}{\partial N u(\operatorname{Re}, \boldsymbol{\alpha})} \frac{\partial N u(\operatorname{Re}, \boldsymbol{\alpha})}{\partial m_{a}} \frac{\partial m_{a}}{\partial D_{f a n}}-\frac{H\left(m_{a}, \boldsymbol{\alpha}\right)}{m_{a}{ }^{2}} \frac{\partial m_{a}}{\partial D_{f a n}}\right] \\
-\frac{\left(m_{w}^{(i)}-m_{w}^{(i+1)}\right) h_{g, w}^{(i+1)}\left(T_{w}^{(i+1)}, \mathbf{a}\right)}{m_{a}{ }^{2}} \frac{\partial m_{a}}{\partial D_{f a n}} ; \quad \ell=4 ; i=1, \ldots, I ; j=34,
\end{gathered}
$$

where $\frac{\partial H\left(m_{a}, \boldsymbol{\alpha}\right)}{\partial N u(\operatorname{Re}, \boldsymbol{\alpha})}$ and $\frac{\partial N u(\operatorname{Re}, \boldsymbol{\alpha})}{\partial m_{a}} \frac{\partial N u(\operatorname{Re}, \boldsymbol{\alpha})}{\partial m_{a}}$ were defined previously in Equations (A105) and (A24), respectively, while $\frac{\partial m_{a}}{\partial D_{f a n}}$ was defined in Equation (A71).

The derivatives of the air/water vapor energy balance equations [cf. Equations (A10)-(A12)] with respect to the parameter $\alpha^{(35)} \equiv \Delta z_{\text {fill }}$ are as follows:

$$
\frac{\partial N_{4}^{(i)}}{\partial \alpha^{(35)}}=\frac{\partial N_{4}^{(i)}}{\partial \Delta z_{f i l l}} \equiv a_{4}^{i, 35}=0 ; \quad \ell=4 ; i=1, \ldots, I ; j=35 .
$$

The derivatives of the air/water vapor energy balance equations [cf. Equations (A10)-(A12)] with respect to the parameter $\alpha^{(36)} \equiv \Delta z_{\text {rain }}$ are as follows:

$$
\frac{\partial N_{4}^{(i)}}{\partial \alpha^{(36)}}=\frac{\partial N_{4}^{(i)}}{\partial \Delta z_{\text {rain }}} \equiv a_{4}^{i, 36}=0 ; \quad \ell=4 ; i=1, \ldots, I ; j=36 .
$$

The derivatives of the air/water vapor energy balance equations [cf. Equations (A10)-(A12)] with respect to the parameter $\alpha^{(37)} \equiv \Delta z_{b s}$ are as follows:

$$
\frac{\partial N_{4}^{(i)}}{\partial \alpha^{(37)}}=\frac{\partial N_{4}^{(i)}}{\partial \Delta z_{b s}} \equiv a_{4}^{i, 37}=0 ; \quad \ell=4 ; i=1, \ldots, I ; j=37 .
$$


The derivatives of the air/water vapor energy balance equations [cf. Equations (A10)-(A12)] with respect to the parameter $\alpha^{(38)} \equiv \Delta z_{d e}$ are as follows:

$$
\frac{\partial N_{4}^{(i)}}{\partial \alpha^{(38)}}=\frac{\partial N_{4}^{(i)}}{\partial \Delta z_{d e}} \equiv a_{4}^{i, 38}=0 ; \quad \ell=4 ; i=1, \ldots, I ; j=38 .
$$

The derivatives of the air/water vapor energy balance equations [cf. Equations (A10)-(A12)] with respect to the parameter $\alpha^{(39)} \equiv D_{h}$ are as follows:

$$
\frac{\partial N_{4}^{(i)}}{\partial \alpha^{(39)}}=\frac{\partial N_{4}^{(i)}}{\partial D_{h}} \equiv a_{4}^{i, 39}=\frac{\left(T_{w}^{(i+1)}-T_{a}^{(i)}\right)}{m_{a}} \frac{\partial H\left(m_{a}, \boldsymbol{\alpha}\right)}{\partial D_{h}} ; \quad \ell=4 ; i=1, \ldots, I ; j=39,
$$

where $\frac{\partial H\left(m_{a}, \alpha\right)}{\partial D_{h}}$ was defined in Equation (A145).

The derivatives of the air/water vapor energy balance equations [cf. Equations (A10)-(A12)] with respect to the parameter $\alpha^{(40)} \equiv A_{\text {fill }}$ are as follows:

$$
\frac{\partial N_{4}^{(i)}}{\partial \alpha^{(40)}}=\frac{\partial N_{4}^{(i)}}{\partial A_{\text {fill }}} \equiv a_{4}^{i, 40}=\frac{\left(T_{w}^{(i+1)}-T_{a}^{(i)}\right)}{m_{a}} \frac{\partial H\left(m_{a}, \boldsymbol{\alpha}\right)}{\partial A_{f i l l}} ; \quad \ell=4 ; i=1, \ldots, I ; j=40,
$$

where $\frac{\partial H\left(m_{a}, \alpha\right)}{\partial A_{\text {fill }}}$ was defined in Equation (A147).

The derivatives of the air/water vapor energy balance equations [cf. Equations (A10)-(A12)] with respect to the parameter $\alpha^{(41)} \equiv A_{\text {surf }}$ are as follows:

$$
\frac{\partial N_{4}^{(i)}}{\partial \alpha^{(41)}}=\frac{\partial N_{4}^{(i)}}{\partial A_{\text {surf }}} \equiv a_{4}^{i, 41}=\frac{\left(T_{w}^{(i+1)}-T_{a}^{(i)}\right)}{m_{a}} \frac{\partial H\left(m_{a}, \alpha\right)}{\partial A_{\text {surf }}} ; \ell=4 ; i=1, \ldots, I ; j=41,
$$

where $\frac{\partial H\left(m_{a}, \alpha\right)}{\partial A_{\text {surf }}}$ was defined in Equation (A149).

The derivatives of the air/water vapor energy balance equations [cf. Equations (A10)-(A12)] with respect to the parameter $\alpha^{(42)} \equiv \operatorname{Pr}$ are as follows:

$$
\frac{\partial N_{4}^{(i)}}{\partial \alpha^{(42)}}=\frac{\partial N_{4}^{(i)}}{\partial \operatorname{Pr}} \equiv a_{4}^{i, 42}=\frac{\left(T_{w}^{(i+1)}-T_{a}^{(i)}\right)}{m_{a}} \frac{\partial H\left(m_{a}, \alpha\right)}{\partial \operatorname{Pr}} ; \quad \ell=4 ; i=1, \ldots, I ; j=42,
$$

where $\frac{\partial H\left(m_{a}, \alpha\right)}{\partial \operatorname{Pr}}$ was defined in Equation (A151).

The derivatives of the air/water vapor energy balance equations [cf. Equations (A10)-(A12)] with respect to the parameter $\alpha^{(43)} \equiv V_{w}$ are as follows:

$$
\frac{\partial N_{4}^{(i)}}{\partial \alpha^{(43)}}=\frac{\partial N_{4}^{(i)}}{\partial V_{w}} \equiv a_{4}^{i, 43}=0 ; \quad \ell=4 ; i=1, \ldots, I ; j=43 .
$$

The derivatives of the air/water vapor energy balance equations [cf. Equations (A10)-(A12)] with respect to the parameter $\alpha^{(44)} \equiv V_{\text {exit }}$ are as follows:

$$
\begin{gathered}
\frac{\partial N_{4}^{(i)}}{\partial \alpha^{(44)}}=\frac{\partial N_{4}^{(i)}}{\partial V_{\text {exit }}} \equiv a_{4}^{i, 44}=\left(T_{w}^{(i+1)}-T_{a}^{(i)}\right)\left[\frac{1}{m_{a}} \frac{\partial H\left(m_{a}, \boldsymbol{\alpha}\right)}{\partial N u(\operatorname{Re}, \boldsymbol{\alpha})} \frac{\partial N u(\operatorname{Re}, \boldsymbol{\alpha})}{\partial m_{a}} \frac{\partial m_{a}}{\partial V_{\text {exit }}}-\frac{H\left(m_{a}, \boldsymbol{\alpha}\right)}{m_{a}{ }^{2}} \frac{\partial m_{a}}{\partial V_{\text {exit }}}\right] \\
-\frac{\left(m_{w}^{(i)}-m_{w}^{(i+1)}\right) h_{g, w}^{(i+1)}\left(T_{w}^{(i+1)}, \boldsymbol{\alpha}\right)}{m_{a}{ }^{2}} \frac{\partial m_{a}}{\partial V_{\text {exit }}} ; \quad \ell=4 ; i=1, \ldots, I ; j=44
\end{gathered}
$$

where $\frac{\partial H\left(m_{a}, \alpha\right)}{\partial N u(\operatorname{Re}, \alpha)}$ and $\frac{\partial N u(\operatorname{Re}, \alpha)}{\partial m_{a}}$ were defined previously in Equations (A105) and (A24), respectively, while $\frac{\partial m_{a}}{\partial V_{\text {exit }}}$ was defined in Equation (A86). 
The derivatives of the air/water vapor energy balance equations [cf. Equations (A10)-(A12)] with respect to the parameter $\alpha^{(45)} \equiv m_{w, \text { in }}$ are as follows:

$$
\begin{gathered}
\frac{\partial N_{4}^{(1)}}{\partial \alpha^{(45)}}=\frac{\partial N_{4}^{(1)}}{\partial m_{w, i n}} \equiv a_{4}^{1,45}=\frac{h_{g, w}^{(2)}\left(T_{w}^{(2)}, \alpha\right)}{m_{a}}=\frac{a_{1 g} T_{w}^{(2)}+a_{0 g}}{m_{a}} ; \quad \ell=4 ; i=1 ; j=45, \\
\frac{\partial N_{4}^{(i)}}{\partial \alpha^{(45)}}=\frac{\partial N_{4}^{(i)}}{\partial m_{w, i n}} \equiv a_{4}^{i, 45}=0 ; \quad \ell=4 ; i=2, \ldots, I ; j=45 .
\end{gathered}
$$

The derivatives of the air/water vapor energy balance equations [cf. Equations (A10)-(A12)] with respect to the parameter $\alpha^{(46)} \equiv T_{a, \text { in }}$ are as follows:

$$
\begin{gathered}
\frac{\partial N_{4}^{(i)}}{\partial \alpha^{(46)}}=\frac{\partial N_{4}^{(i)}}{\partial T_{a, i n}} \equiv a_{4}^{i, 46}=0 ; \quad \ell=4 ; i=1, \ldots, I-1 ; j=46, \\
\frac{\partial N_{4}^{(I)}}{\partial \alpha^{(46)}}=\frac{\partial N_{4}^{(I)}}{\partial T_{a, i n}} \equiv a_{4}^{I, 46}=C_{p}^{(I)}\left(\frac{T_{a}^{(I)}+273.15}{2}, \alpha\right)+\omega_{i n} \frac{\partial h_{g, a}^{(I+1)}\left(T_{a, i n}, \alpha\right)}{\partial T_{a, i n}} \\
=C_{p}^{(I)}\left(\frac{T_{a}^{(I)}+273.15}{2}, \alpha\right)+\omega_{i n} a_{1 g} ; \quad \ell=4 ; i=I ; j=46 .
\end{gathered}
$$

The derivatives of the air/water vapor energy balance equations [cf. Equations (A10)-(A12)] with respect to the parameter $\alpha^{(47)} \equiv m_{a}$ are as follows:

$$
\begin{gathered}
\frac{\partial N_{4}^{(i)}}{\partial\left(\alpha^{(i)}\right.}=\frac{\partial N_{4}^{(i)}}{\partial m_{a}} \equiv a_{4}^{i, 47}=-\left(m_{w}^{(i)}-m_{w}^{(i+1)}\right) \frac{h_{g, w}^{(i+1)}\left(T_{w}^{(i+1)}, \alpha\right)}{m_{a}{ }^{2}} \\
+\left(T_{w}^{(i+1)}-T_{a}^{(i)}\right)\left[\frac{1}{m_{a}} \frac{\partial H\left(m_{a}, \alpha\right)}{\partial N u(\operatorname{Re}, \boldsymbol{\alpha})} \frac{\partial N u(\operatorname{Re}, \alpha)}{\partial m_{a}}-\frac{H\left(m_{a}, \alpha\right)}{m_{a}{ }^{2}}\right] ; \quad \ell=4 ; i=1, \ldots, I ; j=47,
\end{gathered}
$$

where $\frac{\partial H\left(m_{a}, \boldsymbol{\alpha}\right)}{\partial N u(\operatorname{Re} \boldsymbol{\alpha})}$ and $\frac{\partial N u(R e, \boldsymbol{\alpha})}{\partial m_{a}}$ were defined previously in Equations (A24) and (A105), respectively.

The derivatives of the air/water vapor energy balance equations [cf. Equations (A10)-(A12)] with respect to the parameter $\alpha^{(48)} \equiv \omega_{\text {in }}$ are as follows:

$$
\begin{gathered}
\frac{\partial N_{4}^{(i)}}{\partial \alpha^{(48)}}=\frac{\partial N_{4}^{(i)}}{\partial \omega_{i n}} \equiv a_{4}^{i, 48}=0 ; \quad \ell=4 ; i=1, \ldots, I-1 ; j=48, \\
\frac{\partial N_{4}^{(I)}}{\partial \alpha^{(48)}}=\frac{\partial N_{4}^{(I)}}{\partial \omega_{i n}} \equiv a_{4}^{I, 48}=h_{g, a}^{(I+1)}\left(T_{a, i n}, \alpha\right) ; \quad \ell=4 ; i=I ; j=48 .
\end{gathered}
$$

The derivatives of the air/water vapor energy balance equations [cf. Equations (A10)-(A12)] with respect to the parameter $\alpha^{(49)} \equiv \operatorname{Re}_{d}$ are as follows:

$$
\begin{gathered}
\frac{\partial N_{4}^{(i)}}{\partial \alpha^{(49)}}=\frac{\partial N_{4}^{(i)}}{\partial \operatorname{Re}_{d}} \equiv a_{4}^{i, 49}=\frac{\left(T_{w}^{(i+1)}-T_{a}^{(i)}\right)}{m_{a}} \frac{\partial H\left(m_{a}, \alpha\right)}{\partial N u\left(\operatorname{Re}_{d}, \alpha\right)} \frac{\partial N u\left(\operatorname{Re}_{d}, \alpha\right)}{\partial \operatorname{Re}_{d}} ; \\
\ell=4 ; i=1, \ldots, I ; j=49,
\end{gathered}
$$

where $\frac{\partial H\left(m_{a}, \boldsymbol{\alpha}\right)}{\partial N u(\operatorname{Re} \boldsymbol{\alpha})}$ was defined in Equation (A105) and $\frac{\partial N u(\operatorname{Re}, \boldsymbol{\alpha})}{\partial \mathrm{Re}_{d}}$ was defined in Equation (A93).

The derivatives of the air/water vapor energy balance equations [cf. Equations (A10)-(A12)] with respect to the parameter $\alpha^{(50)} \equiv S c$ are as follows:

$$
\frac{\partial N_{4}^{(i)}}{\partial \alpha(50)}=\frac{\partial N_{4}^{(i)}}{\partial S c} \equiv a_{4}^{i, 50}=0 ; \quad \ell=4 ; i=1, \ldots, I ; j=50 .
$$


The derivatives of the air/water vapor energy balance equations [cf. Equations (A10)-(A12)] with respect to the parameter $\alpha^{(51)} \equiv S h$ are as follows:

$$
\frac{\partial N_{4}^{(i)}}{\partial \alpha^{(51)}}=\frac{\partial N_{4}^{(i)}}{\partial S h} \equiv a_{4}^{i, 51}=0 ; \quad \ell=4 ; i=1, \ldots, I ; j=51 .
$$

The derivatives of the air/water vapor energy balance equations [cf. Equations (A10)-(A12)] with respect to the parameter $\alpha^{(52)} \equiv N u$ are as follows:

$$
\frac{\partial N_{4}^{(i)}}{\partial \alpha^{(52)}}=\frac{\partial N_{4}^{(i)}}{\partial N u} \equiv a_{4}^{i, 52}=\frac{\left(T_{w}^{(i+1)}-T_{a}^{(i)}\right)}{m_{a}} \frac{\partial H\left(m_{a}, \boldsymbol{\alpha}\right)}{\partial N u} ; \quad \ell=4 ; i=1, \ldots, I ; j=52,
$$

where $\frac{\partial H\left(m_{a}, \boldsymbol{\alpha}\right)}{\partial N u(\operatorname{Re}, \boldsymbol{\alpha})}$ was defined in Equation (A105).

\section{References}

1. Cacuci, D.G.; Fang, R. Predictive modelling of a paradigm mechanical cooling tower. I: Adjoint sensitivity model. Energies 2016, 9, 718. [CrossRef]

2. Cacuci, D.G. Predictive modeling of coupled multi-physics systems: I. Theory. Ann. Nucl. Energy 2014, 70, 266-278. [CrossRef]

3. Cacuci, D.G.; Badea, M.C. Predictive modeling of coupled multi-physics systems: II. Illustrative applications to reactor physics. Ann. Nucl. Energy 2014, 70, 279-291. [CrossRef]

4. Latten, C.; Cacuci, D.G. Predictive modeling of coupled systems: Uncertainty reduction using multiple reactor physics benchmarks. Nucl. Sci. Eng. 2014, 178, 156-171. [CrossRef]

5. Cacuci, D.G.; Arslan, E. Reducing uncertainties via predictive modeling: FLICA4 calibration using BFBT benchmarks. Nucl. Sci. Eng. 2014, 176, 339-349. [CrossRef]

6. Arslan, E.; Cacuci, D.G. Predictive modeling of liquid-sodium thermal-hydraulics experiments and computations. Ann. Nucl. Energy 2014, 63, 355-370. [CrossRef]

7. Aleman, S.E.; Garrett, A.J. Operational Cooling Tower Model (CTTool V1.0); Savannah River National Laboratory: Savannah River, SC, USA, January 2015.

8. Garrett, A.J.; Parker, M.J.; Villa-Aleman, E. 2004 Savannah River Site Cooling Tower Collection; SRNL-DOD-2005-07; Atmospheric Technologies Group; Savannah River National Laboratory: Aiken, SC, USA, 2005.

9. Cacuci, D.G.; Ionescu-Bujor, M. Model calibration and best-estimate prediction through experimental data assimilation: I. mathematical framework. Nucl. Sci. Eng. 2010, 165, 18-44. [CrossRef]

10. Cacuci, D.G.; Ionescu-Bujor, M. On the evaluation of discrepant scientific data with unrecognized errors. Nucl. Sci. Eng. 2010, 165, 1-17. [CrossRef]

11. Faragó, I.; Havasi, A.; Zlatev, Z. Advanced Numerical Methods for Complex Environmental Models: Needs and Availability; Bentham Science Publishers: Oak Park, IL, USA, 2013.

12. Cacuci, D.G.; Navon, M.I.; Ionescu-Bujor, M. Computational Methods for Data Evaluation and Assimilation; Chapman \& Hall/CRC: Boca Raton, FL, USA, 2014.

13. Cacuci, D.G. Second-order adjoint sensitivity analysis methodology (2nd-ASAM) for computing exactly and efficiently first- and second-order sensitivities in large-scale linear systems: I. Computational methodology. J. Comput. Phys. 2015, 284, 687-699. [CrossRef]

14. Cacuci, D.G. Second-order adjoint sensitivity analysis methodology (2nd-ASAM) for large-scale nonlinear systems: I. Theory. Nucl. Sci. Eng. 2016, in press.

15. Cacuci, D.G. Second-order adjoint sensitivity analysis methodology (2nd-ASAM) for computing exactly and efficiently first- and second-order sensitivities in large-scale linear systems: II. Illustrative application to a paradigm particle diffusion problem. J. Comput. Phys. 2015, 284, 700-717. [CrossRef]

16. Cacuci, D.G. Second-Order Adjoint sensitivity and uncertainty analysis of a benchmark heat transport problem: I. Analytical results. Nucl. Sci. Eng. 2016, 183, 1-21. [CrossRef] 
17. Cacuci, D.G.; Ilic, M.; Badea, M.C.; Fang, R. Second-order adjoint sensitivity and uncertainty analysis of a benchmark heat transport problem-II: Computational results using G4M reactor thermal-hydraulics parameters. Nucl. Sci. Eng. 2016, 183, 22-38. [CrossRef]

18. Cacuci, D.G. Second-order adjoint sensitivity analysis methodology (2nd-ASAM) for large-scale nonlinear systems: II. Illustrative application to a paradigm nonlinear heat conduction benchmark. Nucl. Sci. Eng. 2016, in press. [CrossRef]

(C) 2016 by the authors; licensee MDPI, Basel, Switzerland. This article is an open access article distributed under the terms and conditions of the Creative Commons Attribution (CC-BY) license (http://creativecommons.org/licenses/by/4.0/). 